\title{
AN EXPERIMENTAL MODEL TO MIMIC THE MECHANICAL BEHAVIOR OF A SCAFFOLD IN A CARTILAGE DEFECT.
}

\author{
Ph. D Thesis by \\ Line Vikingsson
}

\author{
to obtain the Doctor degree \\ at the Universitat Politècnica de València \\ Valencia (Spain), June 2015
}

Thesis Supervisors:

Dr. José Luis Gómez Ribelles

Dr. Gloria Gallego Ferrer 


\section{Preface}

This thesis is divided into different parts: First the hypothesis and objectives are presented, to be followed by introduction and the materials and methods part. The proceeding chapters describe each part of the research with corresponding results and discussion. The thesis is completed with general conclusions and future studies.

The introduction presents articular cartilage and describes the biomechanics of the joint. The current state of tissue regeneration is portrayed, such as the clinical strategies for cartilage repair.

The material and methods part gives the details of experimental techniques and biomaterials used throughout the research.

The chapters dedicated to research results are based on studies previously published or under revision for publishing. In each chapter a brief state of art introduces the study, followed by results, discussion and conclusions.

Chapter three introduces an experimental model as artificial cartilage mimic. The model resembles the in vivo conditions of a porous scaffold implanted in a cartilage defect, by a Poly(Vinyl Alcohol) hydrogel. The model is applied on a Polycaprolactone scaffold and the mechanical properties of the scaffold/hydrogel 
construct are evaluated.

Chapter four applies the experimental cartilage model on a biostable acrylic scaffold series with different crosslinking density, and evaluates the mechanical properties of the scaffolds. The influence of different grades of crosslinking density on the mechanical properties of the scaffold, as well as the effect of water in the porous scaffolds is evaluated.

Chapter five deepens the understanding of the influence of microand macro porosity on the mechanical properties of porous scaffolds. The study compares the influence of micro-porosity and hydrogel density on the mechanical behavior of the porous scaffold/hydrogel construct.

Chapter six describes a fatigue study of the PCL/PVA construct, under physiological conditions up to $100 \quad 000$ cycles of unconfined compression cycles. The morphology and mechanical properties are evaluated after each cycle of fatigue to follow the course of the compression impact on the scaffold and hydrogel.

Chapter seven describes the design of a biomedical device for cartilage repair. The design is based on a patent for a biomedical implant device for cartilage repair, and the study is adapted to be designed for animal studies with a thin cartilage surface. The 
device is designed and implanted in two sheep models, for two different kind of implants.

\section{Acknowledgments}

This thesis is owed to many persons. First of all, I would like to express my deepest gratefulness to my supervisors, Dr. Jose Luis Gómez Ribelles and Dr. Gloria Gallego Ferrer. Jose Luis, for believing in me and giving me the opportunity to stay and work at the Center of Biomaterials. Both of you for encouraging me throughout these years and always supporting me in the daily work. Your great support has been both professional and far beyond what is expected in the role as supervisor. It's a great pleasure to work under supervision and at the same time have liberty to explore and take own decisions. This thesis has been carried out with the help of national projects MAT2010-21611C03-01 and MAT2013-46467-C4-1-R. I performed an exchange stay of one month at the Universidade do Minho through concept of proof project INNOVA 1201-SP20120373, which also financed a part of the research performed in this thesis. I was fortune to enjoy a two months secondment at BioElpida Company in Lyon through project Marie Curie Actions by the FIBROGELNET project PIAP-GA-2012-324386. My acknowledgment to Dr. Senenxtu 
Lancaros at the Faculty of Physics at the Universidade do Minho, Braga in Portugal for receiving me and giving me professional support during my stay in Braga. I would like to thank Eric Garcia and Dr. Benoit Pinteur at BioElpida, Lyon in France for such a nice welcoming in Lyon, and teaching me about GMP conditions and sharing their experiences. My appreciation also goes to Dr. Jose Antonio Gómez-Tejedor for always taking time to help and teach me about mechanical properties, and Dr. Jorge Más Estellés for valuable time and help with the fatigue experiments. I am thankful to Dr. Ana Vallés Lluch for taking me under her wings and introducing me to the world of Biomaterials and Tissue Engineering. Thanks to her I found the great world of biomaterials and got inspired to continue researching. I want to thank Dr. Carmen Carda and Maria Sancho-Tello at the Facultat de Medicina i Odontologia at the Universitat de València for their work with the in vivo studies, Dr. Juan Carlos Monllau, Hospital de la Santa Creu I Sant Pau, and Dr. Santos Martinez Diaz at the Universitat Autònoma de Barcelona for professional work with the implantation of the scaffolds. I am also grateful to Ricardo Pérez Feito for his help with custom-made devices for the mechanical assays. Any great achievement is due to a great team behind and CBIT is no exception, why I would also like to thank the rest of the laboratory members for all time spend, the nice company and always any help needed. Specially to Laura, whose 
incredible patience, organization and kindness saved many experiences and valuable time. My gratitude is extended to my graduate students Babeth, Marta and Alvaro for their contributions and great work. At last, my thankfulness to my family for their unconditional love, support and for making it possible to find my way and study abroad. 


\section{Index}

AN EXPERIMENTAL MODEL TO MIMIC THE MECHANICAL BEHAVIOR OF A

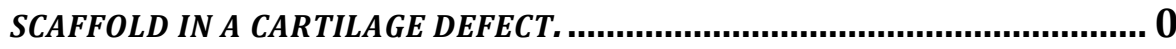

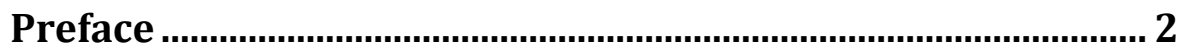

Acknowledgments........................................................................ 4

Index

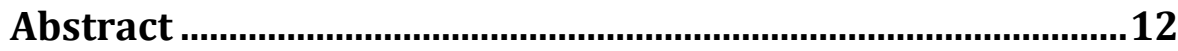

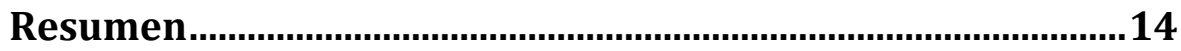

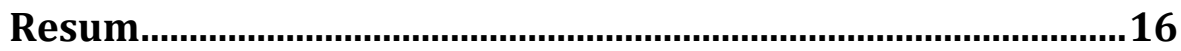

Hypothesis .................................................................................... 18

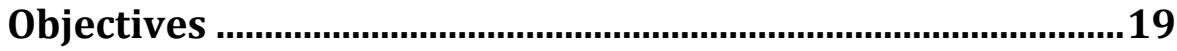

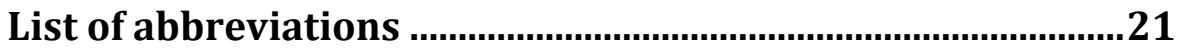

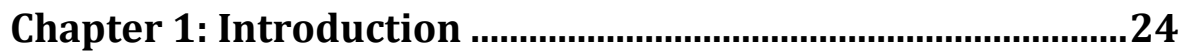

Cartilage development ................................................................ 24

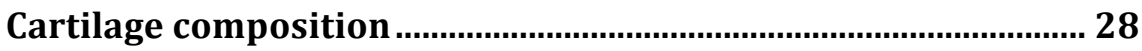

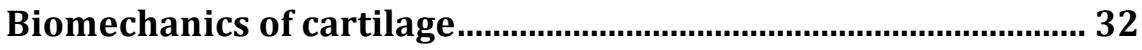

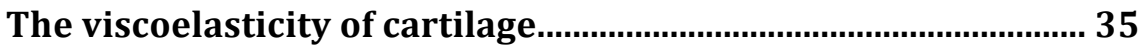

The permeability of articular cartilage .......................................... 38

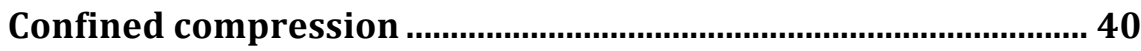

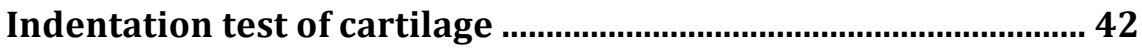

Osteoarthritis and mechanical failure of cartilage ........................ 44

Clinical strategies for regeneration of articular cartilage in the

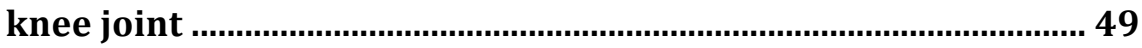

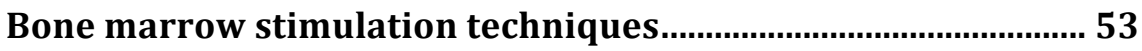

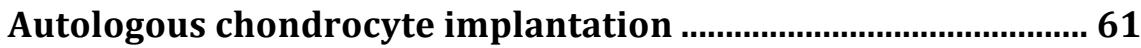

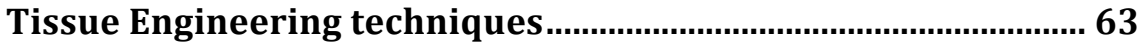

Tissue Engineering Future Challenges.......................................... 70 


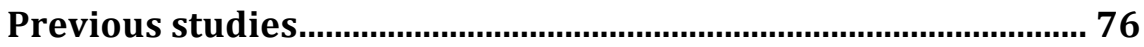

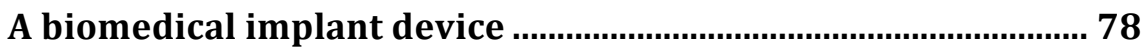

Experimental techniques to evaluate scaffold efficiency ............ 81

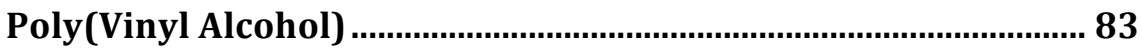

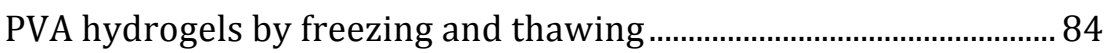

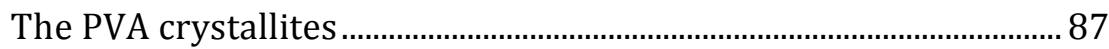

Morphology and pore size........................................................................ 91

Water in the hydrogel ........................................................................ 92

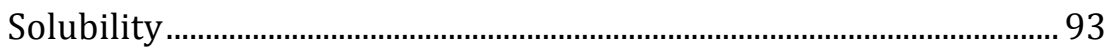

Mechanical properties ......................................................................... 94

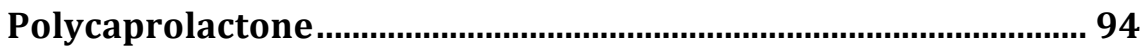

Copolymer networks of Ethyl Acrylate and 2-Hydroxyethyl

Acrylate with varying crosslinking density .................................... 97

Chapter 2: Materials and Methods ......................................... 100

PCL Scaffold preparation ............................................................... 100

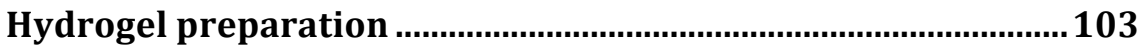

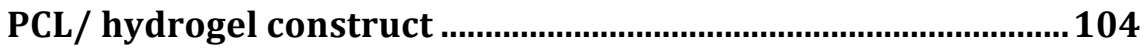

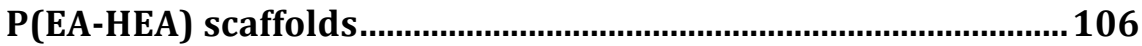

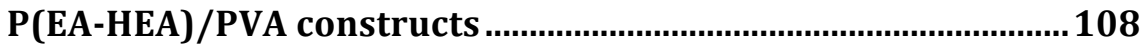

Unconfined compression .............................................................

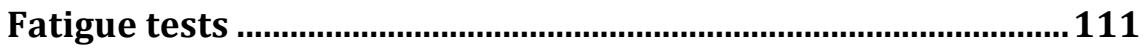

Confined compression ................................................................112

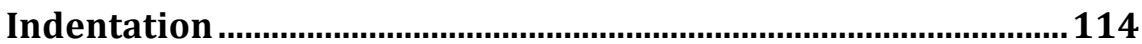

Chapter 3: An in vitro experimental model to predict the mechanical behavior of macroporous scaffolds implanted in articular cartilage ............................................................... 116

Results and Discussion ....................................................................117

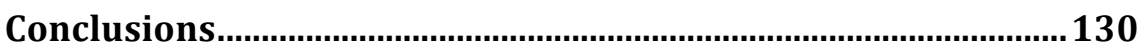

Chapter 4: Prediction of the in vivo mechanical behavior of biointegrable acrylic macroporous scaffolds ..................... 133

Results and Discussion .................................................................134

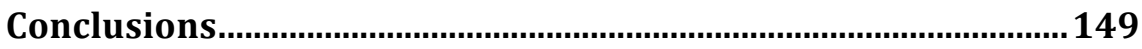


Chapter 5: Relationship between micro-porosity, water permeability and mechanical behavior of scaffolds for cartilage engineering................................................................ 151

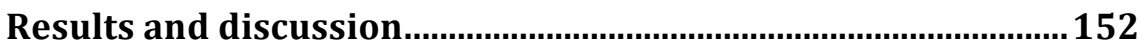

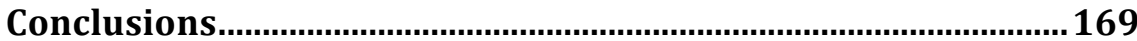

Chapter 6: An experimental fatigue study of a porous scaffold for the regeneration of articular cartilage ............................. 172

Results and discussion.................................................................173

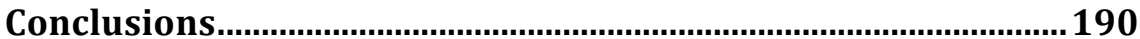

Chapter 7: The design of a biomedical implant device for articular cartilage regeneration ............................................. 192

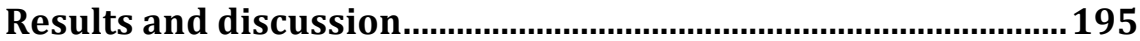

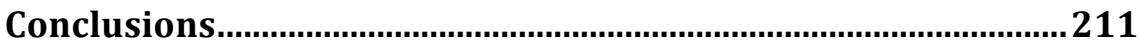

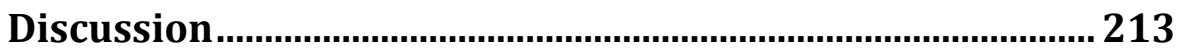

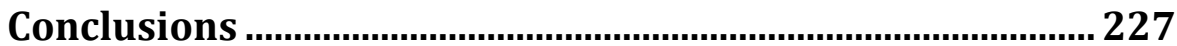

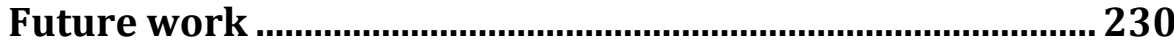

Publications ................................................................................... 232

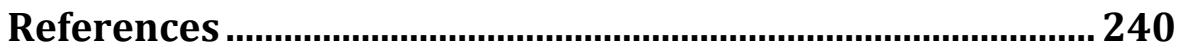




\section{Abstract}

The main purpose of this thesis is the design and characterization of an experimental articular cartilage model. The in vitro model is composed of a macro and micro- porous Polycaprolactone scaffold with a Poly(Vinyl Alcohol) filling. The scaffold/hydrogel construct has been subjected to repeating number of freezing and thawing cycles in order to crosslink the hydrogel inside the scaffold's pores. The Poly(Vinyl Alcohol) resembles the growing cartilaginous tissue inside the scaffolds pores, as it gets denser and stiffer for each cycle of freezing and thawing. The in vitro model allows studying a variety of characteristics of the scaffold and hydrogel, revealing interesting features. The importance of water flow on the mechanical properties is studied, so as the influence of micro-porosity. It can be seen that the mechanical properties of the porous scaffolds are influenced in distinct ways by the hydrogel density and micro-porosity of the scaffold. The permeability of the scaffolds is studied and is seen independent of crosslinking density of the hydrogel inside the porous scaffolds. The experimental cartilage model has also been applied on a macro porous acrylic scaffold. The results show that the water has different effect on the mechanical properties, for macro, or macro and micro-porous scaffolds. The in vitro cartilage model has elastic modulus, aggregate modulus and permeability values 
in the same order as human articular cartilage. The model is useful to predict the mechanical behavior of porous scaffolds in vivo. A scaffold implant device for animal studies has been designed based on a previous patent of the research group, and implanted in two different in vivo trials in sheep. The results show that the fixation and anchoring to the subchondral bone improve the tissue repair and diminish alterations in the subchondral bone. 


\section{Resumen}

El objetivo principal de esta tesis doctoral es el diseño y caracterización de un modelo de cartílago articular experimental. El modelo in vitro se compone de un scaffold micro- $\mathrm{y}$ macroporoso de Policaprolactona con un relleno de Poli(Vinil Alcohol). El constructo scaffold/hidrogel ha sido sometido a ciclos consecutivos de congelación y descongelación con objeto de entrecruzar el hidrogel dentro de los poros del scaffold. El Poli(Vinil Alcohol) mimetiza al tejido de cartílago que se regenerará en los poros, ya que en cada ciclo de congelación y descongelación se vuelve más denso y duro. El modelo in vitro permite estudiar una gran variedad de características del scaffold e hidrogel, revelando fenómenos interesantes para la ingeniería tisular. Se ha estudiado la importancia del flujo de agua a través del scaffold en las propiedades mecánicas, así como la influencia de la microporosidad. Se ha podido constatar que la densidad del hidrogel y la microporosidad influyen de distinta forma en las propiedades mecánicas de los scaffolds porosos. Se ha estudiado la permeabilidad de los scaffolds, que ha resultado ser independiente de la densidad de entrecruzamiento del hidrogel dentro de sus poros. El modelo experimental de cartílago se ha 
aplicado también a un scaffold macroporoso acrílico. Los resultados muestran que el agua tiene un efecto distinto en las propiedades mecánicas de los scaffolds macroporosos y en los micro- macroporosos. El modelo de cartílago in vitro tiene valores del modulo elástico, módulo agregado y permeabilidad que son del mismo orden de magnitud que los del cartílago articular humano. El modelo permite predecir el comportamiento mecánico in vivo de scaffolds porosos. Se ha diseñado un dispositivo de implante de scaffold para experimentos en animales basado en una patente del grupo de investigación, que ha sido implantado en dos ensayos in vivo diferentes en ovejas. Los resultados muestran que la fijación y anclaje al hueso subcondral tiene un gran papel en la reparación del tejido. 


\section{Resum}

L'objectiu principal d'aquesta tesi doctoral és el disseny i caracterització d'un model de cartílag articular experimental. El model in vitro es compon d'un scaffold micro- i macroporós de Policaprolactona amb un farciment de Poli(Vinil Alcohol). El constructe scaffold/hidrogel ha estat sotmès a cicles consecutius de congelació i descongelació amb l'objectiu d'entrecreuar l'hidrogel dins del porus del scaffold. El Poli(Vinil Alcohol) mimetitza al teixit de cartílag que es regenerarà en el porus, ja que en cada cicle de congelació i descongelació es torna més dens i dur. El model in vitro permet estudiar una gran varietat de característiques del scaffold i hidrogel, posant de manifest fenòmens interessants per a l'enginyeria tissular. S'ha estudiat la importància del flux d'aigua a través del scaffold en les propietats mecàniques, així com la influència de la microporositat. S'ha pogut constatar que la densitat de l'hidrogel i la microporositat influeixen de distinta manera en les propietats mecàniques dels scaffolds porosos. S'ha estudiat la permeabilitat dels scaffolds, que ha resultat ser independent de la densitat d'entrecreuament de l'hidrogel dins dels seus porus. El model experimental de cartílag s'ha aplicat també a un scaffold macroporós acrílic. Els resultats mostren que l'aigua té un efecte distint en les propietats 
mecàniques dels scaffolds macroporosos i en els micromacroporosos. El model de cartílag in vitro té valors del mòdul elàstic, mòdul agregat i permeabilitat que són del mateix ordre de magnitud que els del cartílag articular humà. El model permet predir el comportament mecànic in vivo de scaffolds porosos. S'ha dissenyat un dispositiu d'implant de scaffold per a experiments en animals basat en una patent del grup d'investigació, que ha segut implantat en dos assaigs in vivo diferents en ovelles. Els resultats mostren que la fixació i ancoratge a l'os subcondral té un gran paper en la reparació del teixit. 


\section{Hypothesis}

We hypothesize that the long-term performance of a scaffold implanted in a chondral defect can be predicted by means of measurements conducted "ex vivo" in an experimental model. This model consists of filling the scaffold with a gel that simulates the growing repair tissue within the scaffolds pores. The model should allow the prediction of mechanical properties, such as compliance, water permeability and fatigue resistance.

Regarding clinical solutions for cartilage regeneration, we believe that the anchoring system of the scaffold to the subchondral bone is an important factor for tissue repair. We believe that a given scaffold device, designed for humans, can be adapted to animal models in such way that the experiments can evaluate the stability of the implant. 


\section{Objectives}

The main objective of this thesis study is to contribute to the development of scaffold implants for articular cartilage repair with tissue engineering techniques. The clinical solutions offered today mostly generate a fibrous cartilaginous tissue. We believe that scaffolds for cartilage repair need mechanical improvement and subchondral bone anchoring, to improve the tissue repair. Hereof, the study mostly concentrates on porous scaffolds made of biodegradable Polycaprolactone. Previous developments of our group demonstrated the suitability of this material for cartilage engineering. The objective however, is also to obtain results that can be applied on other scaffolding systems. Therefore, a biostable scaffold system made of Poly(Ethyl Acrylate) and Poly(2- Hydroxyl Ethyl Acrylate) has been evaluated. The scaffolds implanted in articular cartilage must create an adequate biomechanical environment for cells, and thus prediction of the mechanical behavior of the scaffold implanted in the joint is essential. The material is expected to reside in the implantation site for a long time, subjected to dynamic compression. It is important to have a tool to ensure that the implant is able to sustain the long term loading. Therefore, this study wants to find means to test the mechanical properties of the porous scaffold in 
simulated in vivo conditions, when the pores are filled with physiological fluids and growing repair tissue. The specific objectives are therefore to develop an experimental model that simulates the growing tissue inside the scaffolds pores. With this model we want to study:

- How the in vivo time-dependent tissue repair can be simulated experimentally

- How macro and micro porosity influence the mechanical behavior and permeability of porous scaffolds

- The fatigue behavior of porous scaffolds and the influence of hydrogel filling

- The differences in mechanical response of scaffolds with double pore architecture to scaffolds with solely macroporosity

Furthermore, the objective of this study is to contribute to the clinical solutions for cartilage regeneration offered today, and to their improvement. This is made by designing a biomedical device for implantation in the articular cartilage. The design is based on a patented scaffold device, and adapted to animal models. The objectives is to assess the stability of the implant, and evaluate the importance of subchondral bone anchoring on tissue repair. 


\section{List of abbreviations}

ACI-Autologous Chondrocyte Implantation

AMIC-Autologous Matrix-Induced Chondrogenesis

bMSC-bone Marrow derived Mesenchymal Stem Cell

hMSC-human bone Marrow derived Mesenchymal Stem Cells

BMP-Bone Morphogenetic Protein

Cryo-SEM-cryogenic Scanning Electron Microscopy

DSC-Differential Scanning Calorimetry

EA-Ethyl Acrylate

ECM-Extra Cellular Matrix

EGDMA-Ethylene Glycol Dimethylacrylate

EtOH-Ethanol

FDA- Food and Drug Administration (US)

F/T-Freezing and Thawing

FGF-Fibroblast Growth Factor $\beta 1$

GAG-Glycosaminoglycan

HE-Hematoxylin and eosin

HEA-Hydroxyethyl Acrylate 
MRI-Magnetic Resonance Imaging

TE-Tissue Engineering

TGA-Thermo Gravimetric Analysis

PCL-Polycaprolactone

PEA-Poly(Ethyl Acrylate)

P(EA-HEA)-Poly(Ethyl- 2-Hydroxyl Ethyl Acrylate)

PEG-Poly(Ethylene Glycol)

PEMA- Poly (Ethyl Methacrylate)

PHEA-Poly(2-Hydroxyl Ethyl Acrylate)

PLA-Poly(L-lactic acid)

PMMA-Poly(Methyl Methacrylate)

PRP-Platelet Rich Plasma

PVA-Poly(Vinyl alcohol)

SEM-Scanning Electron Microscopy

OA-Osteoarthritis 
Introduction

\section{Chapter 1: Introduction}

\section{Cartilage development}

Cartilage is a connective tissue found in different parts of the body. The three mayor types of cartilage are:

- Elastic cartilage found in ears, epiglottis and larynx

- Fibrous cartilage found in intervertebral discs, articular joints and ligament

- Hyaline cartilage found in craniofacial structures, trachea and growth plate of long bones and surface of joint articulations.

Articular hyaline cartilage is an avascular tissue with relatively few cells, called chondrocytes. The special arrangements of cells and extra cellular matrix components in hyaline cartilage make it a highly specialized tissue with load bearing capacities and low friction properties. The articular cartilage in the knee joint covers the long bones femur and tibia, plus the patella (Figure 1). 


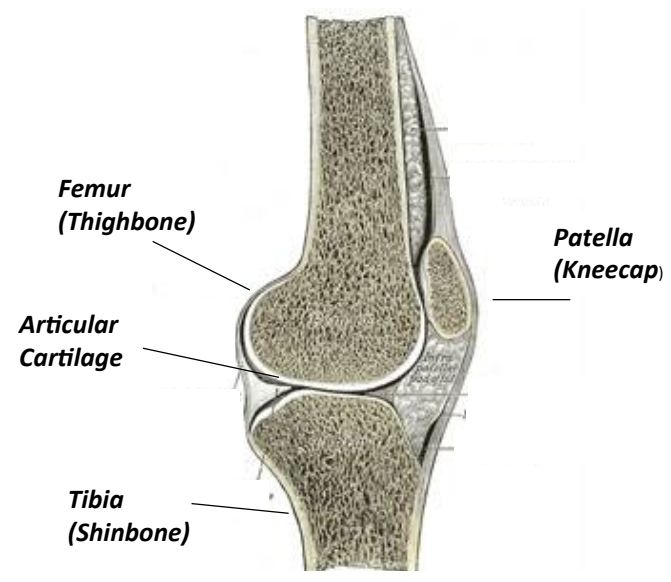

Figure 1. The anatomy of the knee joint seen from the side. The articular cartilage is seen as a white surface. (1)

Development of cartilage, also called chondrogenesis, is the earliest phase of skeletal development. The skeletal system is derived from the mesoderm germ layer during embryogenesis. Chondrogenesis is a dynamic cellular process during vertebral development and leads to the different cartilage types. During limb development and growth, chondrocytes form cartilage at the end of long bones. Chondrogenesis form cartilage intermediate and leads to endochondral ossification during bone and 
Introduction

skeletal development. This process is initiated by mesenchymal cell recruitment and migration, proliferation and condensation into precartilage (Figure 2).

(2) Chondrogenesis is dependent and regulated by cell-cell and cell-matrix signals and is seen as increased cell adhesion and changes in cytoskeletal architecture.

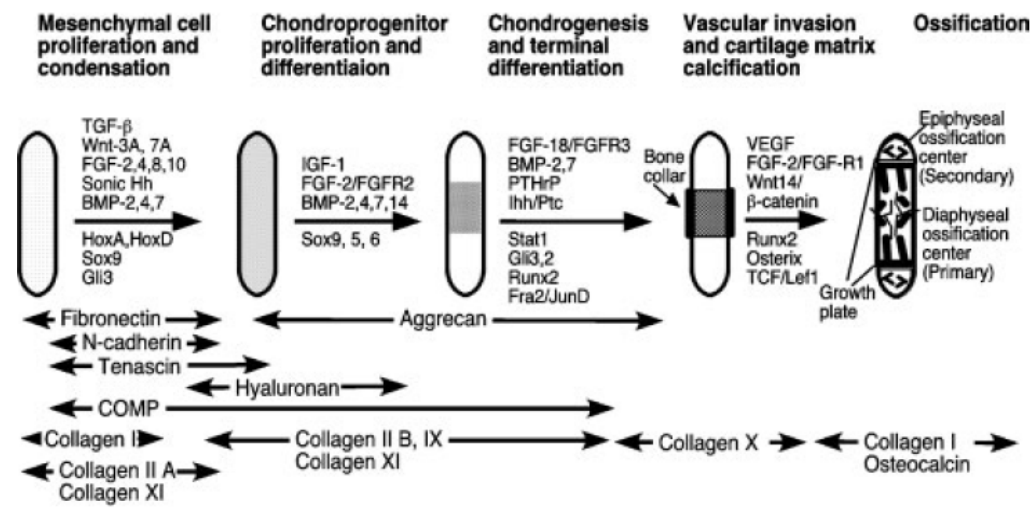

Figure 2. Chondrogenesis during endochondral ossification. (3)

Briefly, chondrogenesis and endochondral ossification are initiated by mesenchymal cell condensation to progenitor cells that differentiate to chondroblasts. Endochondral ossification begins with formation of periosteum in a primary ossification center. The periosteum contains undifferentiated cells that differentiate to osteoblasts. The osteoblasts form bone collar in appositional growth of the bone shaft. The chondrocytes in the primary center of 
ossification begin to proliferate and undergo hypertrophy. The chondrocytes cease the secretion of collagen and proteoglycan and start secreting alkaline phosphatase, an important enzyme in mineral deposition. The chondrocyte matrix then calcifies, and osteoprogenitor cells use the calcified matrix for bone trabecular formation. The bone marrow is formed by osteoclasts by breakdown of spongy bone. The calcified cartilage is then resorbed and replaced by bone. Chondrocytes are in this way pushed to the articular surface of the long bones and form the thin articular cartilage layer. This is how chondrocytes form the articular cartilage at the end of opposing bones, with the cartilage zones formed during the process. The limb development is controlled by interacting pathways involving fibroblast growth factor, FGF, transforming growth factor $\beta 1$, and bone morphogenetic protein, BMP. Interactions with positive and negative signaling kinases and transcription factors, such as Sox9 and Runx2 control the destiny of differentiated chondrocytes. (3) (4) (5) 
Introduction

\section{Cartilage composition}

Hyaline cartilage is composed of cells, chondrocytes, and extra cellular matrix, ECM, produced by chondrocytes. Chondrocytes make up less than $5 \%$ of the total tissue volume. A healthy hyaline cartilage consists of $70-75 \%$ water, up to $20 \%$ proteoglycans, and $10-20 \%$ collagen. (6) (7)(8) Chondrocytes do not have any direct cell to cell contact and are enclosed in ECM cavities called lacunae. Proteoglycans consist of a core protein with covalently attached glycosaminoglycans, GAG, chains that are long linear negatively charged carbohydrate polymers. The most abundant proteoglycan in cartilage is aggrecan, also called cartilage-specific-proteoglycan core protein. Aggrecan is a high molecular weight proteoglycan with a bottlebrush structure in which the negatively charged chondroitin sulfate and keratin sulfate chains are attached to a protein core (Figure 3). (9) (10) (11) Hyaluronic acid is another important component in the cartilage ECM and unlike the proteoglycans, not attached to a core protein. It is an anionic nonsulfated glycosaminoglycan that is found in abundance in cartilage. It is a polysaccharide consisting of D-glucuronic acid and $\mathrm{N}$-acetylglucosamine residues. Hyaluronic acid is a very hydrophilic molecule and can absorb great quantities of water and contribute to the 
sponge effect of cartilage. Consequently hyaluronic acid is also important in load-bearing in the articular joint. (12) (13)

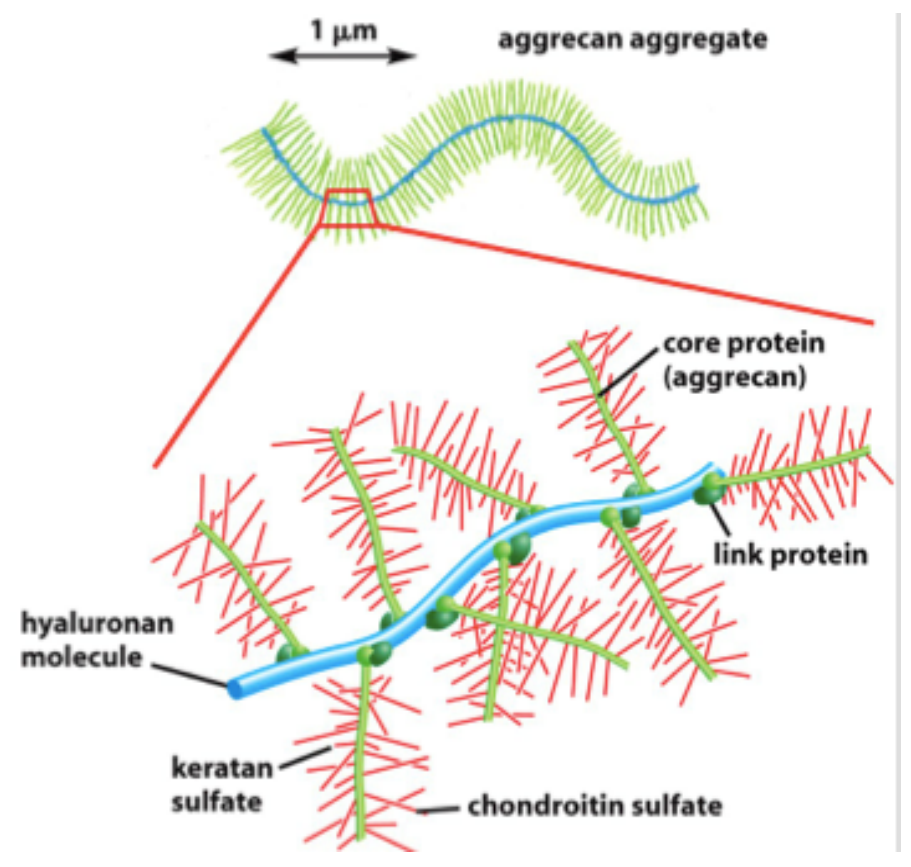

Figure 3. The aggrecan molecule. The molecule consists of a link protein attached to hyaluronan and glycosaminoglycans (keratin and chondroitin sulfate) attached to a core protein. (14) 


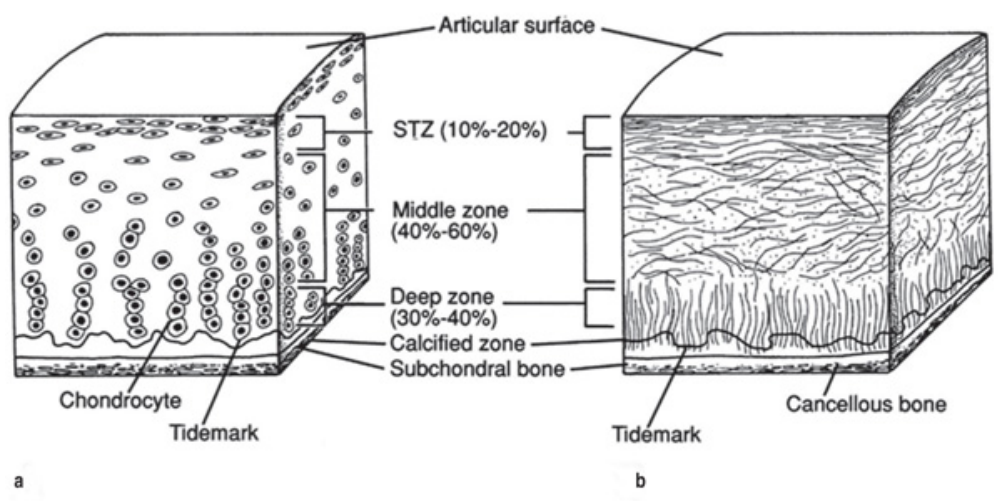

Figure 4. The articular cartilage with different zones. a) Shows the distribution of chondrocytes and b) the collagen fiber alignment. (10)

The other main constituent of cartilage is collagen, that gives strength and elasticity to the tissue. Collagen fibers account for about two thirds of the dry weight of healthy adult cartilage. In mammalian articular cartilage the collagen types vary with age: from fine fibrils with about $80 \%$ collagen type II, $10 \%$ collagen IX and $10 \%$ collagen XI to adult thick collagen fibers with more than $90 \%$ collagen type II and about $1 \%$ collagen IX and 3\% collagen XI. (15) The different collagen types IX, II and XI are subunits of the same fibril network (16) and their spatial assembly differ with tissue depth. Chondrocytes and ECM components are distributed in such ways that the tissue is divided into different zones with different characteristics (Figure 4). A healthy articular cartilage in the human knee 
joints is up to $3 \mathrm{~mm}$ thick with four different zones, starting from the surface down to the subchondral bone. (17) The superficial zone represents about $20 \%$ of the tissue and chondrocytes are positioned parallel to the surface with collagen fibers packed in thin bundles in the same direction. This zone of cartilage contains the highest amount of collagen type II and the lowest amount of GAGs. The collagen fibrils are about 200 microns in this zone. (18) (19) Due to this arrangement the superficial zone of cartilage has high tensile strength, withstand great shear stresses and lowers the friction in the joint. The superficial zone has the lowest water content of the tissue. (20) The next zone is the middle, or transitional zone, and it constitutes about $40-60 \%$ of the tissue thickness. The chondrocytes are spherical and the collagen fibers are thicker and both are randomly distributed in this zone. When cartilage is compressed, the collagen fibers in this zone reorient towards a horizontal position which helps resist compression loads. Proteoglycans are abundant and reach their maximum level in the middle zone. The next zone is the deep zone where chondrocytes are rounded and aligned in vertical columns, so as the collagen fibers. The deep zone has the highest level of GAGs and the highest elastic modulus. In the interface with the subchondral bone the calcified cartilage layer is 
Introduction

characterized by hypertrophic chondrocytes and mineralized tissue. This zone has a higher level of collagen X. (21) (22) (17) (23) (24)

\section{Biomechanics of cartilage}

The elastic modulus of human articular cartilage in compression is normally between $0.5-0.8 \mathrm{MPa}$ (25) (26) and increases with tissue depth from the articular surface. (27) Cartilage normally withstand physiological frequencies between 0.1 and $10 \mathrm{~Hz}$. (28) (29) (30) The high elastic modulus is a consequence of the water swelling capacities of negatively charged GAGs and collagen fiber alignment. (31) Proteoglycans are polyanionic in aqueous solutions, due to the sulfate and carboxyl groups in the glycosaminoglycan chains. When compression loading is applied to the joint and cartilage is compressed, the proteoglycans negative charges are pushed close together, which increases the mutual repulsive forces and makes cartilage a hard tissue. Cartilage is in this way a sponge, with proteoglycans absorbing great amounts of water. The mechanical modulus of cartilage increases with the glycosaminoglycan content. The proteoglycans are entrapped in collagen 
fibers (Figure 5) and the volume of proteoglycans is therefore limited by entanglements of collagen. (31) (20)

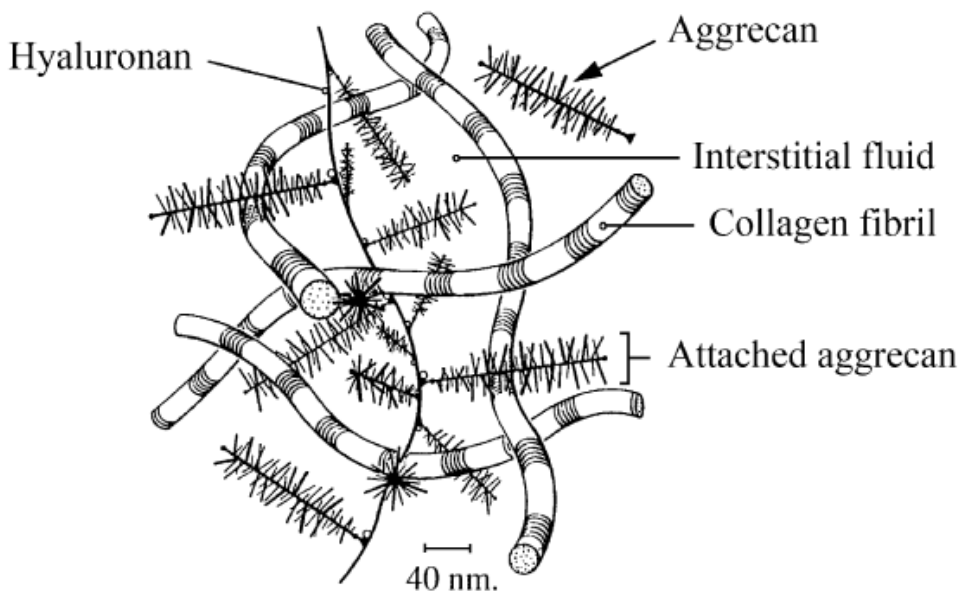

Figure 5. The collagen and proteoglycan network interact and form a porous solid matrix in cartilage. (32)

Not only the proteoglycans but also the collagen fibers play an important role in the mechanical properties of cartilage. If the collagen framework is damaged or reduced, the proteoglycans are less entrapped and loose their aggregation capacity. (33) (33) The shear deformation is mostly dependent on collagen, involving predominantly sliding of collagen microfibrils. (34) When cartilage is stretched in tension the collagen fibers are stretched. The collagen fibrils and entangled proteoglycan aggregates align and stretch in the loading direction. The 
stiffness of the collagen fibers in turn depends on the orientation and density of the fibers and grade of crosslinking. (35) The tensile modulus is considered an intrinsic, flow-independent property of cartilage and in the order of 5-25 MPa in human joints. (35)

In the superficial zone the collagen fibers make the tissue resistant to shear stresses and it is also the zone where highest strains (up to 50\%) can be reached. This is where practically all fluid flow out of the tissue and this diminishes the hydrostatic pressure. The axial compressive modulus is lower in the superficial zone. In the deeper zones the fluid flow is practically zero, giving a high hydrostatic pressure and low strains. (27) The differences in tensile and compressive strength of the cartilage zones have important influences on the deformation characteristics under load. (36) (26) The main mechanism that makes chondrocytes receive nutrients are based on the diffusion across the articular surface. Nutrients from the synovial fluid diffuse through the articular surface and reach chondrocytes in the tissue. (37) (38) (39) 


\section{The viscoelasticity of cartilage}

The articular cartilage is as stated composed of a solid matrix which primarily consists of negatively charged proteoglycans, collagen fibers and water. The proteoglycans create a swelling pressure, giving compressive resistance to the tissue. The collagen fibers resists the swelling and determine the tensile properties. The articular cartilage is structurally inhomogeneous and possesses anisotropic and nonlinear mechanical characteristics. To get close to reality, articular cartilage has been modeled with biphasic, triphasic and elastic models. (25) Most models consider the tissue as a homogeneous and isotropic material, even though more realistic, and complex, models have been developed. The commonly accepted methods to determine the mechanical properties of cartilage are unconfined compression, confined compression and indentation testing. (32)

As discussed, the compressive stiffness of cartilage is highly dependent on fluid flow through the tissue and the articular surface. (20) When cartilage is deformed, fluid flows through the tissue and the articular surface, making the tissue behaving somehow like a sponge. Because of the composition of cartilage with cells, solid extra cellular 
matrix molecules and interstitial fluid that flows though the solid parts it is common to treat cartilage as a biphasic material. The biphasic model considers cartilage as a solid part (ECM molecules) and a fluid part (interstitial fluid). (40) (41) (42) The solid part is considered an isotropic, linearly elastic solid and the fluid part as an incompressible material without viscosity. The forces acting within cartilage during load are, according to the biphasic model: pressures acting within a deformed network of proteoglycans and collagen, frictional drag between the ECM molecules and water, and pressure within the fluid phase. (40) These three internal force responses give cartilage viscoelastic properties under load, meaning that the load response will differ with time. Under fast load fluid does not have time to leave the tissue fast enough, and gives an elastic solid response. The pressurized fluid components give cartilage its loadbearing capacities and viscoelastic properties. (43) The interstitial fluid between the organic matrix molecules and the friction raised is governed by tissue permeability, and provides a significant energy dissipation mechanism. This is seen in creep and stress relaxation tests when a constant load or a constant strain is applied to the tissue, and fluid exudates through the articular surface and is redistributed inside the tissue. (44) (45) (40) The biphasic 
theory considers cartilage as a solid porous elastic part and a fluid part, but in reality there is another additional factor to consider, the fixed charge density. As mentioned, there are more complex models to describe the articular cartilage tissue. A triphasic theory has been developed, including the biphasic system with solid-fluid phases and additionally an ion phase, to describe the stress and strain response for cartilage under mechanical load. (46) The proteoglycans in the ECM of cartilage are negatively charged due to the sulfate $\left(\mathrm{SO}_{3}{ }^{-}\right)$and carboxyl groups ( $\mathrm{COO}^{-}$) at the glycosaminoglycans. The negative charges results in a high charge density in cartilage, and generates an imbalance of mobile ions across the articular surface of cartilage to the synovial fluid. This gives rise to a pressure difference that generates a higher fluid pressure within the cartilage, named Donnan osmotic pressure. (47) From these phenomena two definitions emerge: the intrinsic and the apparent properties. The biphasic model is considered to give rise to apparent properties, and the triphasic model gives intrinsic properties. The Donnan osmotic pressure is shown to contribute to $30-50 \%$ of the equilibrium compressive stiffness of cartilage. Swelling of cartilage then depends on the fixed charge density, the collagen and proteoglycan matrix and the ion concentration in the synovial fluid. The triphasic model of 
cartilage assumes constant fixed charges and counter ions in the synovial fluid as cations of a single salt. The gradients of the chemical potentials are the driving force for movement and depend on fluid pressure, salt concentration, solid matrix swelling and fixed charge density. (48) (49)

\section{The permeability of articular cartilage}

The hydraulic permeability is an important parameter to describe the flow-dependent behavior of cartilage in compression. The apparent permeability of articular cartilage is in the order of $10^{-15} \mathrm{~m}^{4} / \mathrm{Ns}$ and is nonlinearly dependent of tissue compression. (41) (50) Permeability can be described as a measurement of the internal frictional resistance of ECM molecules to interstitial flow. The low permeability value of cartilage reflects that both great interstitial fluid pressures and energy dissipations occur inside the tissue. The permeability of cartilage is inversely related to the negative charges of the proteoglycans. The proteoglycan content is increasing with tissue depth and so the fixed charge density. The permeability is decreasing with tissue depth and reaches the lower values in the deep zone, where the proteoglycans are abundant and the load is the highest. 
The cartilage surface is an exception, since the surface permeability is very low. (51) (52) Cartilage permeability also varies with the tissue deformation. When the tissue is compressed, the permeability decreases, which will make fluid flow decrease. Regarding permeability through the cartilage zones, there exist different interpretations of the phenomena across the cartilage and bone interface. Some researchers claim that there is no exchange of water or solutes between the cartilage and the bone (51) (53) (54), whereas other authors have seen a passage of molecules from both the subchondral bone and from the articular surface to the articular cartilage. (55)(56)(57) An accepted theory today seems to be that the calcified cartilage is permeable from the surface and only in some extend from the subchondral bone, and foremost in younger persons. (43)(58)(59) As mentioned, the permeability at the cartilage surface is very low. The collagen fibers are smaller and closely packed at the surface of cartilage, which creates a system with narrow channels and greater flow resistance. (60) (61) (52) The close weave of small collagen fibrils at the cartilage surface could have several functions. It gives cartilage a smooth wear resisting surface and energy dissipation characteristics. Whereas the deeper zones can absorb more energy upon deformation, the superficial zone can 
release this energy when unloaded. It also serves as filter for bigger molecules, preventing loss of important extra cellular matrix components and hindering synovial molecules to enter cartilage. (51)

Permeability can be measured directly from the relationship between fluid pressure and interstitial fluid flux (60) or by mathematical models from flow-dependent strain or stress response compression tests. By measuring the compressive modulus when the fluid flow cease at equilibrium the solid matrix stiffness will be reflected without the influence of interstitial fluid flow and fluid pressurization. (40)

\section{Confined compression}

In confined compression the load is applied to the cartilage specimen through a porous plate where fluid can flow out. By confined compression tests the permeability and aggregate modulus of cartilage can be measured. As previously described, permeability is the resistance to fluid flow through the cartilage solid matrix. The average fluid velocity through the tissue is proportional to the pressure gradient applied, and permeability is the proportionality constant. In confined compression tests, the cartilage is placed in an impervious fluid filled well, so 
fluid can only flow out through the porous loading plate. By confined compression test, either the load or the strain applied can be constant. If the load applied is constant and strain is measured with time, the experiment is called creep. If the strain is constant and force measured with time, the experiment is called stress relaxation test. In creep, cartilage is deformed under constant load but the deformation is not instantaneous, and depends on fluid flow out of the tissue. The initial displacement is fast, with a large fluid flow out of the cartilage. The interstitial fluid immediately pressurizes upon confined compression loading and make up more than $95 \%$ of the total loadbearing. (62) The rate of displacement slow down with time and the strain reaches a constant value. When equilibrium is reached the strain is constant and the fluid flow deceases. In stress relaxation tests, the strain is hold constant and force measured in time. The force required to hold the strain at a certain level will diminish with time as the extracellular matrix reorganize in the tissue. The force will eventually be constant and the system reaches equilibrium when fluid flow stops. The aggregate modulus is the mechanical stiffness at equilibrium when the fluid flow has stopped. In cartilage the aggregate modulus is in range of 0.5-0.9 $\mathrm{MPa}$. (63) 
Introduction

\section{Indentation test of cartilage}

Indentation is the most common experimental method to calculate the biomechanical properties of cartilage. Indentation differs from normal compression in the way that the compression pole used is only one fifth of the sample diameter. The cartilage specimen is tested with the subchondral bone intact under the cartilage and resembles the in vivo situation. (64) Indentation is the most convenient experimental method to determine the mechanical properties of cartilage, as it can be done on specimens with different size and shape. The common cartilage model is a biphasic isotropic homogeneous linear elastic solid phase with an incompressible, inviscid fluid model. The single phase linear elastic model is an approximation of the cartilage mechanical response and used for short time response or long time equilibrium response. The parameters in a linear elastic assumption are Young's modulus and Poisson's ratio. Young's modulus is the stress-strain coefficient in the linear elastic zone. When a material is compressed or stretched in one direction, it expands or stretches in the opposite direction. Poisson's ratio is the negative ratio of transverse to axial strain. Hooke's law is another proportional constant, saying that for small deformations the displacement is 
proportional to the load. (65) (66) (67) (68) Indentation or confined compression tests can be used to determine the material constants by the biphasic model. When the Poisson's ratio is calculated for a material according to the biphasic model, it is calculated in equilibrium state. In the equilibrium state, the water flow has ceased in the material and the aggregate modulus, Poisson's ratio and relaxation time can be calculated. The short time response is then assigned to the viscoelastic solid and the long time response to the biphasic fluid flow. (69) To calculate the mechanical behavior from indentation tests Hayes model can be used. (Equation 1) (67) The model searches Young's modulus $\left(E_{s}\right)$ that is determined from the slope $(E)$ of the equilibrium stress-strain curve. The model then associates the slope with the Poisson's coefficient $(v)$, the indentation radius $(a)$, the height of the specimen $(h)$ and $k$ which is a function of specimen volume and Poisson's coefficient.

$$
E_{s}=E \frac{\left(1-v_{2}^{2}\right) \pi a}{2 k h}
$$


This model assumes totally elastic behavior of the material and is therefore valid when the load only just started, for short times assuming there is not other contributions, and for infinitive long times, in which the time dependent deformations already occurred.

\section{Osteoarthritis and mechanical failure of cartilage}

Osteoarthritis, OA, or degenerative joint disease, is one of the most common skeletal disorders and characterized by cartilage degradation. The articular cartilage has limited regeneration capacity and $\mathrm{OA}$ disables approximately $10 \%$ of the first world population over 60 years old. Fullthickness chondral defects are more common among athletes with a high level of mechanical compression loading on the joints, than among the rest of the population. (70) Cartilage degradation can be seen as cracks in the articular surface, fibrillation and loss of tissue (Figure 6). There can also be signs of subchondral bone changes, joint space narrowing, increased cartilage hydration and altered cellular activity together with structural and organizational changes of cartilage composition. (71) (72) (73) Some theories say that degeneration starts with vertical cracks initiated by high tensile stresses on the articular surface. (74) It is still 
unclear about the exact molecular mechanism behind OA, but it is believed that $\mathrm{OA}$ is induced by accumulated mechanical stress. It might sound rare since previously stated that mechanical loading affects chondrocyte differentiation positively (75) and increases ECM production (37), but one should remember this is always moderate physiological deformations. High mechanical loading has the reverse effect. It is not clear how ever, if the tensile stress, the compression loading or the shear stresses on cartilage are the predominant factors provoking fibrillation and cracking of the surface. Repeated high compressive loads lead to a decrease in tensile strength, which may imply that internal damage in the tissue occur before apparent surface fibrillation. The compressive modulus decrease with increasing severity of degradation in the joint. (76) The tensile modulus decrease up to $90 \%$ in degenerative joints which show irreversible changes to extra cellular matrix components, in particular the collagen fibers. The collagen fibrillar network suffers a disorganization, rupture, unwinding, fibrillation or loss of crosslinking which lowers the tensile strength. (77) (26) Other factors as gender, obesity, trauma and occupation have shown to influence both the mechanical stress and the OA directly. (78) 

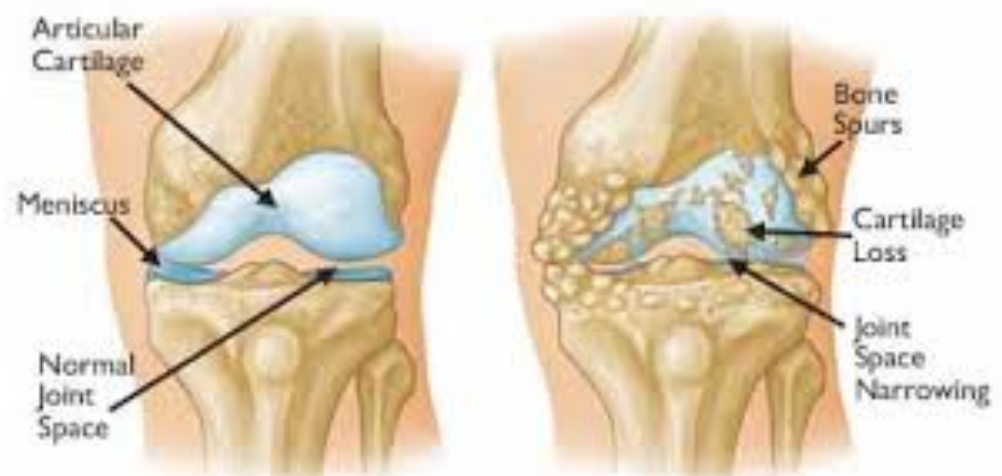

Figure 6. Illustration of a normal joint and a joint with OA. (79)

In the healthy joint ECM molecules like collagen type II, proteoglycans and hyaluronic acid make up a framework that supports the compressive forces exerted in the joint. The mechanical properties of cartilage are due to water binding characteristics of GAGS and elastic collagen fibers. In a joint with $\mathrm{OA}$ the proteoglycans are fragmented and often found in the synovial fluid. The mechanical loading then is subjected directly to the collagen fibers that are destroyed and degraded. There are several upstream signals that can cause degradation of cartilage, starting from mechanical failure (Figure 7). For example matrix metalloproteinases and aggrecanases, are shown to be initiators of $\mathrm{OA}$ progression. These proteinases damage the core protein of proteoglycans. (80) (81) The proteinases are in turn induced by different factors and 
signals. The fragments of degraded proteins are also shown to trigger additional degradation. Proinflammatory factors produced by chondrocytes and synovial cells may also contribute to the progression of OA. Mechanical stress can induce transcriptional activator Runx2, that affects chondrocyte hypertrophy and chondrocyte apoptosis. Those steps in the endochondral ossification are seen to be important for the progression of OA. Regarding the onset of $\mathrm{OA}$, cartilage degradation may start in the center of the knee joint by the activation of proteinases. The proteinase activation can in turn be due to hypertrophic chondrocytes. In the edge of the joint vascularization is increased and the same signals can cause osteophyte formation. In the center of the joint there is no vascularization from synovium, why cartilage is degraded without being replaced by bone. (82) 


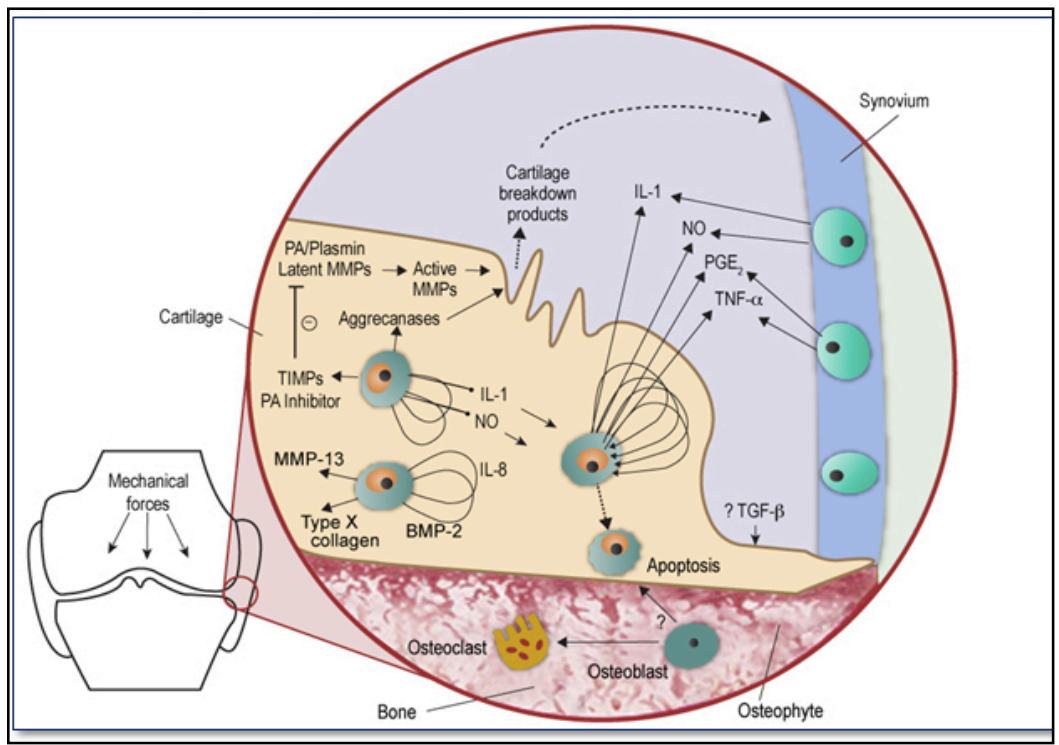

Figure 7. Initiators and pathways of OA in the knee joint. (83)

As previously described normal joint function depends on absorption and transmission of loads by the healthy articular cartilage. In normally functioned joint, articular cartilage will provide lubrication, energy dissipation and load bearing capacities. As previously described, the loadbearing capacity of cartilage is a result of interstitial fluid flow through a porous solid matrix, and physical interactions between ECM components that results in water swelling capacity. The load response is viscoelastic, and hence varies with time, and so gives rise to stress relaxation, creep and energy dissipation effects. If cartilage is damaged, either by injury or degeneration of ECM components, the mechanical function of the joint will 
decrease, which can lead to further degeneration of the tissue. Small changes in the composition or organization of the ECM will lead to changes of the mechanical properties and function of the joint. Another consequence of cartilage degradation is tissue swelling. When proteoglycan entanglement in the collagen fibers are damaged or the collagen fibers disorganized, the water swelling capacities increase. As more water can flow through the ECM, permeability increases and fluid can flow out of the tissue easily which increase the rate of deformation. (8) (73) (84)

Clinical strategies for regeneration of articular cartilage in the knee joint

As stated, the articular cartilage has limited capacity to regenerate after damage or loss of tissue. This is mainly due to its avascular nature, which implies that no recruiter cells can arrive to the defect. The first step after identifying the defect is classification according to location, grade, size and morphology of the lesion. The decision of which surgery or treatment to chose is made by the surgeon and is based on size and nature of the defect, weighted with the patients age, physical condition and expectations. It is also important to find out if the cartilage lesion is caused by trauma or genetics, and if the 
defect is a developing osteoarthritic lesion. (85) Articular cartilage injuries are normally discovered during arthroscopy and diagnostic imaging of the knee joint. (86) The joint can be evaluated by X-rays, spiral CT 3D construction, magnetic resonance imaging, MRI, or single photon emission computed tomography. MRI is still the most efficient and reliable technique to detect superficial articular cartilage lesions. Arthroscopy is however the best tool to evaluate the cartilage defects, with the disadvantage of easily overestimate the cartilage size. There are no strict rules about choosing the best treatment for cartilage defects, current clinical strategies depend not only on the nature of the defect but also on the choice of the surgeon. (85) Not all cartilage defects are treated, since the adjacent native cartilage can suffer from a surgery. On the other hand untreated defects, even small, can progress and cause functional problems leading to pain, swelling and disability. The management and choice of cartilage treatment can be divided into age related treatments. For adolescents, surgery is normally not recommended, as it can interfere with the growth and regeneration potential of the patients own cartilage. Instead non operational treatments are suggested. For low activity patients up to forty-five years old microfracture, described in detail below, is the first choice for full- 
thickness chondral lesions. The patients age has shown to influence highly on the outcome of the microfracture. If the cartilage lesion is contained another treatment option is osteoarticular transfer system, mosaicplasty (Figure 8). This technique uses cartilage from a healthy part of the joint and moves in to the defect, in the form of plugs. (87) If the patient is physically very active the treatment choice might be different. The microfracture may allow faster recovery and successive come-back to sports, but also show tissue deterioration with time. Third generation autologous chondrocyte implantation, ACI, will be described in detail below and is shown to have slower recovery process, but a later similar success ratio in returning to sports. The results in clinical studies also show that ACI might have better long-term results compared to microfracture in high physical activity patients. (88) 


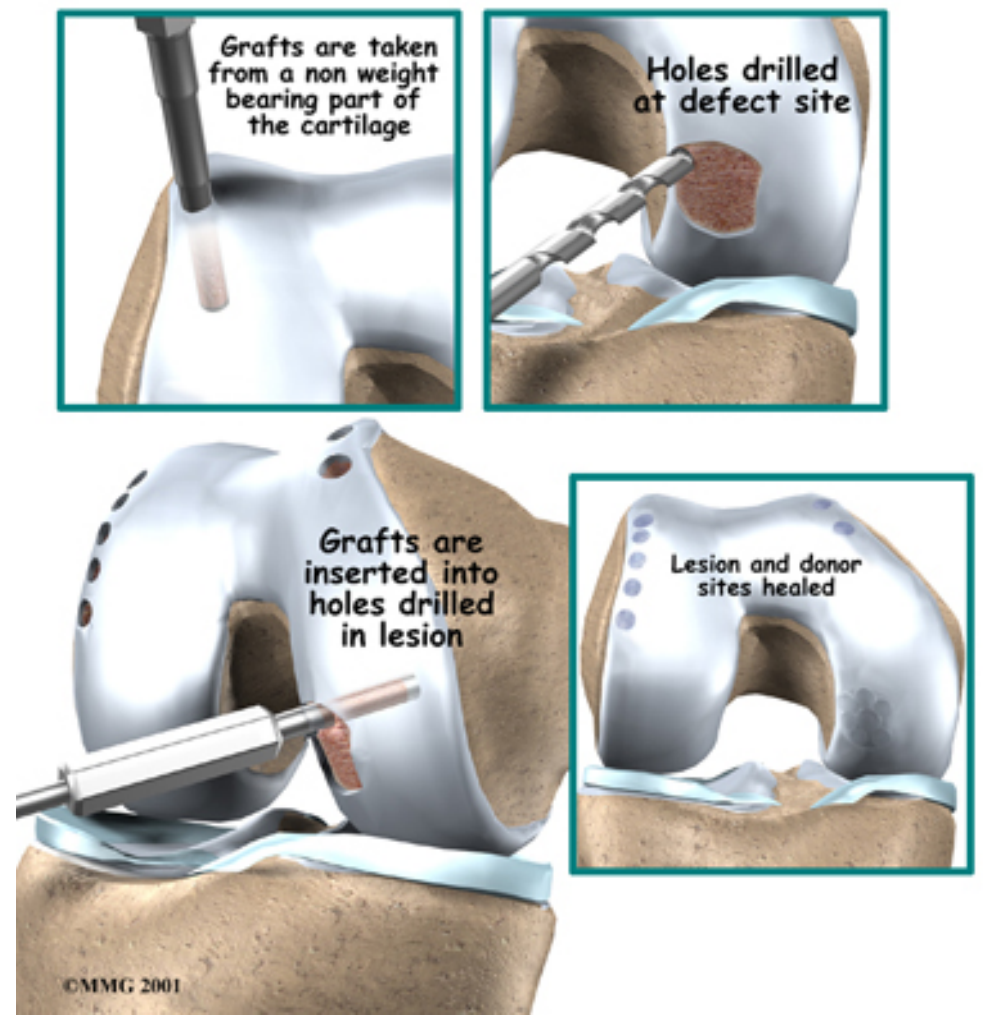

Figure 8. In mosaicplasty fresh cartilage in the form of plugs is taken from a non load-bearing area and grafted into the defect. .(89)

For small defects in patients over forty-five years old microfracture is a good option if the defect is contained. For not contained defects microfracture has shown to fail, possible due to patients slow recovery and decreased metabolic activity of cells. (90) Osteochondral grafts could be the best treatment option for patients over forty five 
years with small cartilage defects. (87) The microfracture is thus recommended for young patients with small or medium sized defects, and have shown failure in the longterm results for defects bigger than $3 \mathrm{~cm}^{2}$. (91) For medium size lesions autologous osteochondral transfer could be the best treatment option. The disadvantages of this technique are low donor site availability and defect size-limits. Matrix-assisted ACI, described below, is not Food and Drug Administration, FDA, approved in the United States but a treatment option in Europe, allowing improvements to some clinical concerns such as cell culture and surgical techniques. Tissue Engineering options with scaffolds, growth factors or cells in combination with one of previously described surgical techniques offers promising results. (85)

\section{Bone marrow stimulation techniques}

Bone marrow stimulation techniques aim at repairing articular cartilage with help of bone marrow mesenchymal stem cells, bMSC, recruited from the subchondral bone. BMSC are pluripotent stem cells and can differentiate into chondrocytes under certain conditions. Pridie drilling and abrasion techniques also take advantage of the pluripotency of adult stem cells in subchondral bone 
marrow. (92) (93) The most common bone marrow stimulation technique is microfracture surgery invented by Steadman et al. in 1994. (94) Damaged cartilage is totally removed and holes are drilled down to the subchondral bone, making sure not damaging the subchondral plate (Figure 9). In the first surgeries the calcified cartilage was left intact, but better results are seen when the defect is abraded down to the subchondral bone (Figure 10). Perforations are made with special awls and as close together as possible, which is 3 to $4 \mathrm{~mm}$ apart to assure that they do not go into each other and provoke bigger holes. These perforations provoke bleeding and release of marrow elements, such as mesenchymal stem cells and growth factors. The defect is soon filled with a fibrin clot. The abraded surface of the subchondral bone is rough and good for clot attachment. It has been reported that pluripotent bone marrow progenitor cells differentiate to chondrocyte-like cells and produce a cartilaginous repair tissue that fills the chondral defect. (95) However, the tissue produced is often fibrocartilaginous with lower mechanical properties than the original tissue. The outcome of microfracture is discussed, but reports overall good results, especially for small $\left(<2 \mathrm{~cm}^{2}\right)$ defects in young active patients. (85) 17 years follow up studies have been made, showing increased 
function and pain relief. (96) It is not unusual to have to repeat the surgical treatment due to tissue repair failure. Microfracture might have been the first choice, and as second surgery ACI is chosen. This is however seen having negative results, due to the previous intervention of subchondral bone. (97)

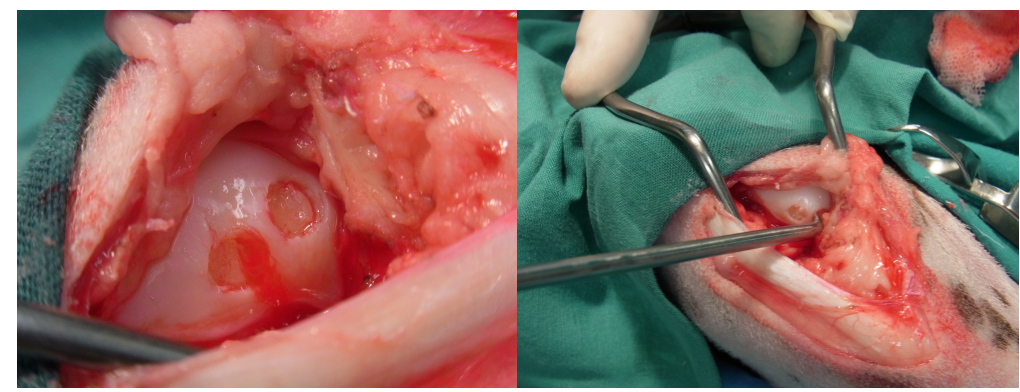

a)

b)

Figure 9. a) The first step during microfracture surgery is to remove damaged cartilage and make a hole down throughout the calcified cartilage to the subchondral bone without interfering with the subchondral plate. b) The second step is to make micro fractures with an awl, allowing bleeding from subchondral bone and the formation of a fibrin clot in the defect. Photo: L.Vikingsson taken during the operations performed by Dr. Santos, further described in chapter seven. 
Introduction

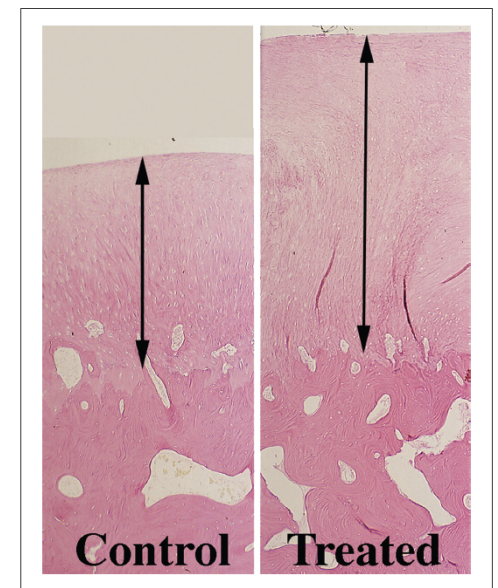

a)

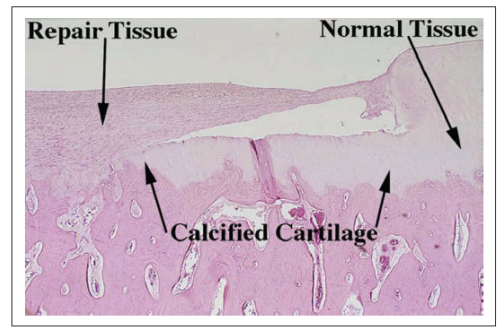

b)

Figure 10. a) An osteochondral sectioned stained with hematoxylin eosin, HE, after microfracture in a horse model. b) It can be seen that the defect is not completely filled and the repair tissue has poor attachment to subchondral bone. The surgery was done in such way that the calcified cartilage was left intact. The results from this study have led to that the microfracture surgery technique changed. (98)

Regarding the tissue produced after microfracture, histological examination in animals show enhanced increase of collagen II expression, but lower aggrecan 
expression. This can be connected with the fibrocartilaginous tissue that has been seen generated after microfracture in humans. Chondrocyte differentiation is to a high extent determined by the mechanical forces acting on the cells during the early stages of differentiation. If the fibrin clot does not offer sufficient mechanical strength in comparison to surrounding tissue, cells will likely not express the markers typically associated with hyaline cartilage, but more for fibrous tissue. As previously described, aggrecan is responsible for the compressive load bearing capacity of cartilage. If the proteoglycan content decreases, so will the mechanical properties.

Cell origin and differentiation in microfracture defects from surgery to tissue growth was investigated with time in a rabbit model. (99) Directly after drilling and during the first hours after surgery red blood cells came to the defect from the vascularized subchondral bone. Fibrin arcades were detected initially across the defect and helped orientating progenitor cells. After one to some few days the fibrin clot got firmer, with fibrinoid material arrangement. New vascularization was seen in the bone marrow. After three days, mesenchymal cells were prominent at the surface and also seen in the deepest zone of the defect. Mesenchymal cells were also seen invading 
the clot from the periphery. Not until one week mesenchymal cells were seen in the middle zone of the clot. After ten days the first signs of aggrecan synthesis were seen. Some days later a fibrous cell layer was seen at the surface. In the deep zone of the defect chondrocytelike cells were forming. In the marrow in the adjacent bone a woven bone was formed. Healthy cartilage adjacent to the defect was seen with empty lacunae, and the cartilaginous tissue close to the defect became necrotic. Two weeks after microfracture the defect was filled with a cartilaginous tissue with flattened fibro-cartilaginous cells. The chondrocyte layer was well developed three weeks posterior surgery. Hypertrophic chondrocytes were detected in the deep zone, bone formation in the depth of the defect and a repair fibrous cartilage tissue filling out the defect. After twelve to twenty-four weeks the cartilage layers were well developed. Tidemark and lamellar subchondral bone plate were well distinguished after three months. Despite many good results seen after microfracture surgery, many defects also showed degradation after three months, with newly synthesized tissue that failed to adhere to adjacent native cartilage. Radioisotope labeling showed that chondrocytes in the adjacent tissue did not participate in the re-population of the defect. It was concluded that the bMSC were 
responsible for the repair tissue formed in the cartilage defect after microfracture. The radioisotope label was also seen in fibroblasts, osteoblasts, articular chondroblasts and chondrocytes. (99)

Autologous matrix-induced chondrogenesis, AMIC, is a modification of the microfracture technique, developed for larger defects and sites difficult to access by ACI method. The subchondral bone is perforated and a collagen membrane is put on top of the defect to maintain the bone marrow blood. The super clot is formed underneath the collagen membrane and induced a fibrous cartilaginous tissue. (100)

Short after invention of the microfracture technique the differentiation potential of bMSC was mapped. (101) Human MSC were isolated from marrow aspirates and the hMSC could be induced to differentiate into adipocytic, chondrocytic and osteocytic lineages (Figure 11). The hMSC developed a proteoglycan and collagen rich matrix after about ten days when cultured with transforming growth factor $\beta 3$. The cells could remain their multilineage potential when expanded to colonies. It was concluded that cell density, nutrients, spatial organization, mechanical forces, growth factors and cytokines had a profound influence on hMSC differentiation. Bone marrow 
Introduction

is the major source of adult hematopoietic stem cells, responsible for renewal of blood elements, and is also a source of adult mesenchymal stem cells. (102)

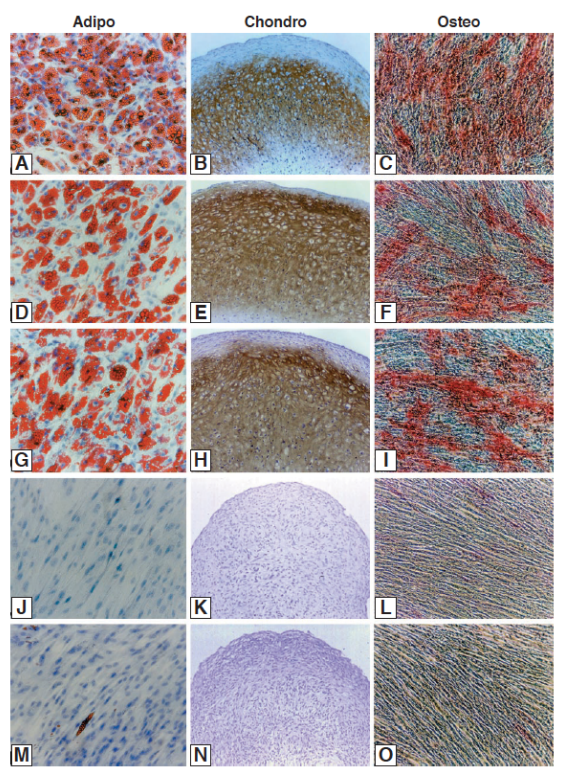

Figure 11. Isolated marrow derived stem cells differentiate into mesenchymal lineages (A-I). The controls from skin fibroblasts (J-G) did not show the same differentiation. Chondrogenesis was detected by staining for collagen II and morphological changes. (101)

It has later been demonstrated that bMSC contribute to the regeneration of mesenchymal tissue such as bone, cartilage, muscle, ligament, tendon, adipose and stroma. Mesenchymal stem cells are also found in other tissue, such as adipose tissue, peripheral blood and placenta and umbilical cord. (102) (103) (104) (105) (106) (107) 


\section{Autologous chondrocyte implantation}

Autologous chondrocyte implantation, ACI, was introduced by Brittberg et al. in 1984. It was the first cartilage surgery using cell engineering techniques. The $\mathrm{ACI}$ is a two step surgery where healthy cartilage is harvested from a less load-bearing area, expanded in culture and then suspended to be injected in the chondral defect (Figure 12). Over the chondrocyte suspension a membrane of periosteum is placed to keep the solution in place. Today most surgeons use the second generation $\mathrm{ACI}$, where the periosteum flap is exchanged by a collagen membrane. Third generation $\mathrm{ACI}$ is the extended version, that consists of autologous chondrocytes seeded in cell carriers or scaffolds and is used widely in clinics. (108) $\mathrm{ACI}$ is normally used as a second surgery option after a first surgery failure. The outcome of ACI is less age-related than microfracture technique, patients over twenty years are reported to have similar results. (109) $2-300 \mathrm{mg}$ $(10 \mathrm{~mm} \times 5 \mathrm{~mm}$ strip$)$ of healthy cartilage is harvested from a low weight bearing zone of the joint, such as the medial femoral condyle or lateral upper femoral trochlea. The patient's blood and the harvested cartilage are sent to cell laboratory for cell expansion and suspension. In the second operation the knee is opened by mini-arthrotomy 
Introduction

and the defect is debrided to leave a clean defect with depth to the subchondral bone, without provoking damaged to the subchondral plate or bleeding. The periosteum flap is harvested from the proximal medial tibia and care is taken to harvest a very thin periosteum without any remaining fibrous tissue. The periosteum flap is sutured over the defect and fibrin glue is used as sealing, and the chondrocyte solution is injected under the flap. Today, most surgeons use a collagen membrane instead of the periosteum flap. (110) Carticel (Genzyme) is the only FDA approved ACI in the US and ChondroSelect (TiGenix) was the first cell-based product approved in Europe.

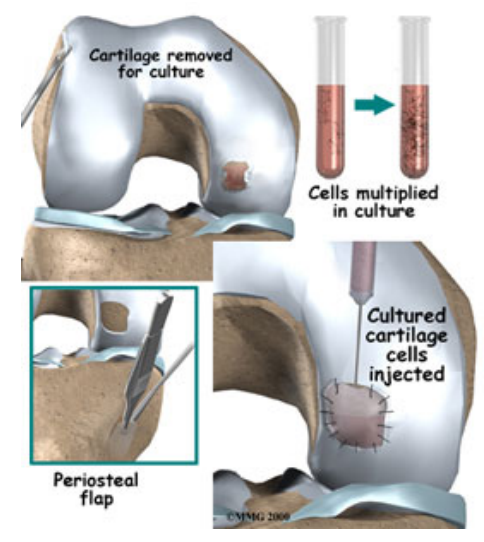

Figure 12. ACI with peropsteal flap, the first autologous chondrocyte implantation technique preocedure invented by Brittberg et al. (89) 


\section{Tissue Engineering techniques}

Tissue Engineering solutions try to find solutions to restore the function of damaged tissue with cells, materials and biological factors. The objective is to regenerate a repair tissue with similar characteristics as the native healthy tissue. Langer and Vacanti started the field of tissue engineering by defining the concept TE as an interdisciplinary field of biology and engineering principles that provides functional solutions for repair damaged tissue. (111)

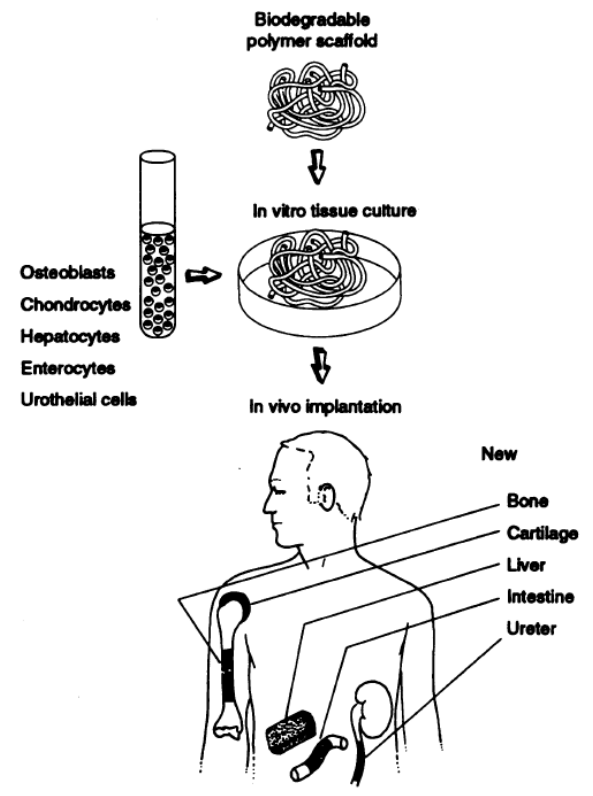

Figure 13. Classical illustration from Langer and Vacanti describing the principles of tissue engineering. (111) 
It is state of art that TE uses at least one of three components: three-dimensional porous scaffolds, cells and signalling factors (Figure 13). The scaffold will in many ways resemble the biphasic structure of natural cartilage with a solid part consisting of polymeric material and newly synthesized tissue, and a fluid part consisting of water and water-soluble substances. It is also commonly accepted that the scaffold should be designed to offer an adequate biomechanical environment for cells and newly formed tissue. The scaffold must sustain initial loads in the tissue and protect cells from excessive forces, i.e. diminishes differences in stress-strain response with respect to neighboring tissue. (112) It is at the same time important that the scaffold permits transmitting appropriate mechanical signals to cells. Cells communicate by mechanotransduction that translates mechanical stimuli to stimulate extra-cellular matrix production. (113) The amount of GAGs produced is directly proportional to the kind of load applied: Static compression of cartilage explants decreases ECM production, while dynamic compression of scaffolds increases ECM production. This effect depends of many parameters, such as strain or frequency. (114) (115) (116) Porosity of the scaffold is important for cell-seeding and ingrowth, and for transport of nutrients and waste 
products. Many experimental studies have explored the in vitro and in vivo behavior of porous scaffolds. (112) (117) (118) (119) Chondrocyte metabolism is dependent on fluid flow, and fluid flow in turn depends on the permeability of scaffold and tissue. (120) It is clear that scaffold porosity, permeability and mechanical strength are important parameters when designing new scaffold structures. Three dimensional scaffolds are often both macro and micro-porous, and the porosity can be optimized to obtain unique mechanical and physical scaffold properties. (121) (122) (123) Micro-porosity is seen to have a limited role in scaffold permeability, why further studies were suggested to evaluate the relationship between porosity, permeability and trabecular interconnectivity. (124) Mechanical properties of the scaffold thus depend on the material, porosity and not to forget, physiologic fluid and cells filling the pores. When characterizing scaffolds for TE it is important to remember that the scaffold does not sustain all the stress alone; in vivo the pore structure is being filled progressively with a tissue that contributes to the mechanical performance of the implant. The mechanical behaviour in vivo is difficult to predict and it is not always possible to measure mechanical properties after sacrifice in animal models. Few studies try to identify cartilage 
Introduction

regeneration with time in porous implants. A bioestable poly(ethyl acrylate-co-hydroxyethyl acrylate) copolymer scaffold was implanted in a full-depth chondral defect with abrasion of the subchondral bone to provoke bleeding. (125) Morphological evolution of cartilage repair was studied at different times after implantation. After one week of implantation a neo-tissue growth was observed in the deepest zone of the scaffold pores, and spread up through the scaffold with time.

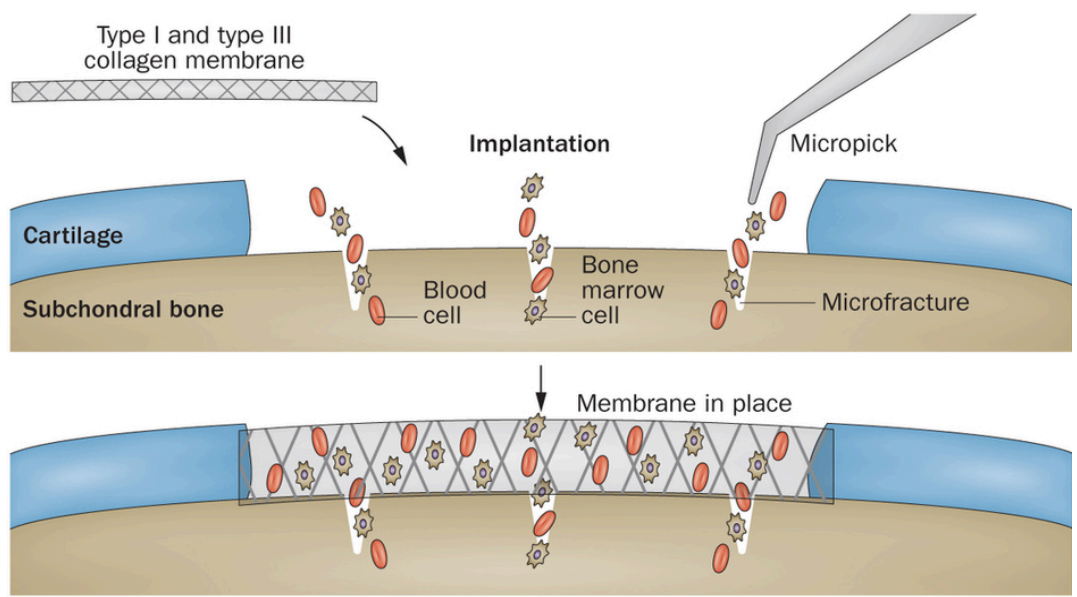

Figure 14. Principles for autologous matrix induced chondrogenesis, AMIC. A collagen scaffold is placed into a microfracture in a fullthickness cartilage defect. (126)

Native chondrocytes at the edge of the defect started to proliferate after one week and after one month the edges had grown into the scaffold. After three months the 
scaffold were filled with cartilaginous tissue and with bony tissue in adjacent subchondral bone. The scaffold was implanted with and without allogenic chondrocytes and results were similar for both implants. After one year the implant were completely covered with a cartilaginous tissue. On the contrary, controls without scaffold showed a fibrous repair tissue. The results show that the scaffolds fulfill an important role as transmitting mechanical stresssignal to the cells.

Some current TE strategies combine microfracture surgery technique with scaffolds (Figure 14), hyaluronic acid, growth factors or cytokine modulation. (127) Scaffolds can be of synthetic or natural origin and made micro and macro-porous and have tailored mechanical and biological properties. Hyaluronic acid fleece and cultivated cells were pioneers in the TE strategies. (128) There exist numerous attempts and reports of natural or synthetic scaffolds in combination with cells and/or growth factors in vitro and in vivo but the overall issue is an fibro-cartilaginous tissue generated, that has less mechanical properties than native hyaline cartilage. Investigation focus on cell-based techniques, using autologous cells, stem cells, and osteochondral/chondral grafts and/or bioactive factors. Many scaffolds are 
Introduction

biphasic constructs, composed of collagen, polymers, chitosan, fibrin, hydrogels etc., many used in combination with microfracture. In continuation some of the commercial available biomaterials will be listed, without pretending to be exhaustive. Chondro-Gide (Geistlich Pharma AG), is a high-strength resorbable bilayered Type I/III porcine collagen membrane, which forms a clot over a microfracture site capturing growth factors and stem cells. Results after two years clinical studies are promising with good outcome. (129) BST-Cargel (Piramal Life Science) is a chitosan-glycerol phosphate scaffold that causes blood coagulation when mixed with autologous blood and placed within a cartilage lesion. Clinical trials have shown improved lesion filling and superior repair tissue, in comparison to microfracture. (130) (131) BioCartilage (Arthrex) is a dehydrated micronized allogenic cartilage scaffold implanted over a microfracture together with platelet rich plasma, PRP. PRP has shown to potentiate the cartilage repair process. (132) GelrinC (Regentis) is a Poly(Ethylene Glycol) hydrogel that can be synthesized with different protein constituents, such as albumin, fibrinogen and gelatin. The hydrogel crosslinks in situ and forms a semi-solid clot by exposure to UV light. The hydrogels show a reduced foreign body reaction. (133) CellCoTec have invented a cell-scaffold-processor for a 
porous

Poly(Ethylene

Oxide)

Terephthalate/Poly(Butylene Terephthalate) scaffold. Bone marrow is extracted from the patient, the scaffold is seeded with cells in the processor and the seeded scaffold will be implanted over a cartilage defect, carved down to the subchondral bone. Results from in vivo studies show a fibrocartilaginous surface repair with proteoglycan-rich domains and subchondral cancellous bone formation. (134) MaioRegen (Finceramica) is a tri-layered scaffold with a surface of collagen type I and a lower layer of magnesium-enriched hydroxyapatite. The middle layer is a mix of the three components. Results from clinical trials show good integration with adjacent tissue and normal knee function after one year. (135) Agili-C (CartiHeal) is a cell-free osteochondral implant of aragonite, the crystal form of calcium carbonate, and hyaluronic acid that show promising results. (136) TruFit $\mathrm{CB}^{\mathrm{TM}}$ (Smith \& Nephew, USA) is a osteochondral scaffold with a Poly(Lactic-coGlycolic Acid)/Poly(Glycolic Acid) chondral part and a calcium sulfate part for the bone. The scaffold has shown early promising results but suffers the disadvantage, as most osteochondral implants, of extended subchondral bone alterations. (137) 
Introduction

\section{Tissue Engineering Future Challenges}

Even though many scaffolds are tested in clinical trials, the key for hyaline cartilage repair is yet to be discovered. Most implants suffer from long-term failure or have similar results as microfracture or ACI controls. The successful scaffold will improve tissue regeneration and most importantly, improve patient life quality after operation. The perfect scaffold should also be user friendly, as surgeons want an easily implanted device. Preferably the operative steps and procedures should be diminished, to reduce operation time and number of interventions. Costs must be reduced, since joint operations are relatively expensive and only implants that are economically competitive will be able to enter public health care. All these factors must be taken in to account when designing, fabricating and manufacturing scaffolds for clinical trials to be commercialized.

So, why are current TE strategies not working today? As mentioned, the regenerated tissue is mainly fibrocartilaginous with less mechanical properties which degrades with time. Hyaline cartilage is not easy to regenerate and the answer may be in the type of cell used, kind of implant or even in the growth factors and signal 
factors used for cell differentiation. Without doubt, there are still many questions to still answer. Chondrogenesis is still not totally understood, and maybe revealing all pathways of chondrogenic differentiation will be the key to understand tissue regeneration and even the onset mechanism of Osteoarthritis. As described in the previous section, there are many different kind of scaffolds available on the market. Generally, they can be divided into porous implants or injectable hydrogels placed above a microfracture surgey, or used in combination with ACI. This thesis focuses on the mechanical properties of scaffolds, believing that the right mechanical load during tissue regeneration is the key for specific cell differentiation. This study also emphasis the importance of the subchondral bone in the repair process. Injectable hydrogels are attractive since they offer an easily manipulated solution. The polymer solution, with or without previously seeded chondrocytes or autologous bMSC, is injected above the cartilage defect that can be a microfracture or chondral defect. The solution is crosslinked in situ and forming a hydrogel with cells inside. (138) (133) (139) (140) The defect can be of any size and shape, facilitating the surgeons work. However, a concern is the low mechanical properties of the hydrogel. During cell differentiation the mechanical load is crucial 
Introduction

and if the load is excessive, in comparison to adjacent tissue, a fibrous tissue is liked to be formed. This is also the case of microfracture surgery, where the blood clot forms a kind of hydrogel for the bMSC. The porous scaffolds used, either of synthetic or natural polymers, suffers a distinct problematic. The scaffold may have better mechanical properties, but must at the same time be able to transmit the right mechanical load to the cells for mechanotransduction. Tissue ingrowth and cell attachment may also be a issue, why some scaffolds are pre-seeded or functionalized with proteins or growth factors. (132) (141) (133) (142) All scaffolds and hydrogels involving cells require two surgeries and time consuming cell harvest, seeding and implantation steps. If the results are not outstanding, it is difficult to convince surgeons and also investors about proceeding with the manufacturing of these implants. The scaffold must also fit the cartilage defect and be maintained in site. Most membranes are cut according to previous MRI or arthroscopic examination, and fixed with a suture or flap. (143) All these steps are time consuming and not very convenient. Some scaffolds focus on a press-fit technique with biodegradable anchoring system, but perforate the subchondral bone to a high extend. This is shown to influence tissue integration and repair negatively. This 
may also be the problem with osteochondral implants. By making a bigger lesion involving the subchondral bone, bad restoration of subchondral bone plate and tissue is seen. (137) (144)

Alteration of the subchondral bone, with cyst formation and other consequences is a common problem observed after microfracture and other subchondral bone intervention surgeries. The awareness of the importance of the role of subchondral bone in pathologic processes after tissue engineering techniques is recently increasing. It is seen that alterations of subchondral bone has important consequences for the healing process over time. The subchondral bone is starting to be seen as part of the repair tissue, and the articular cartilage and supporting bone should be considered a connected osteochondral specimen. (145) (146) The clinical treatment options offered should be focused on restoring not only just the articular cartilage surface, but the entire joint. Alterations caused by biomechanical failure or other OA progression affects the cartilage and the surrounding tissue, thereof also the subchondral bone. Clinical studies have shown subchondral alterations in $30 \%$ of patients treated with microfracture. The alterations are seen as formation of subchondral cysts, intralesional osteophytes and 
thickening of the subchondral bone. (146) (147) If ACI treatment cause alterations of the subchondral bone is not that clear, according to different studies. On one hand, if the repair tissue generated has lower mechanical properties than the adjacent tissue, the load may be deequilibrated and affect the subchondral bone negatively. On the other hand, since the ACI do not perforate the subchondral bone, less alterations are expected. During articular cartilage wear, the tidemark progressively advance up and thinner the articular surface, and thicker the underlying subchondral plate. The viscoelastic cartilage gets thinner and the subchondral plate gets stiffer in the same rhythm, making it more susceptible to compression load and shear forces. 

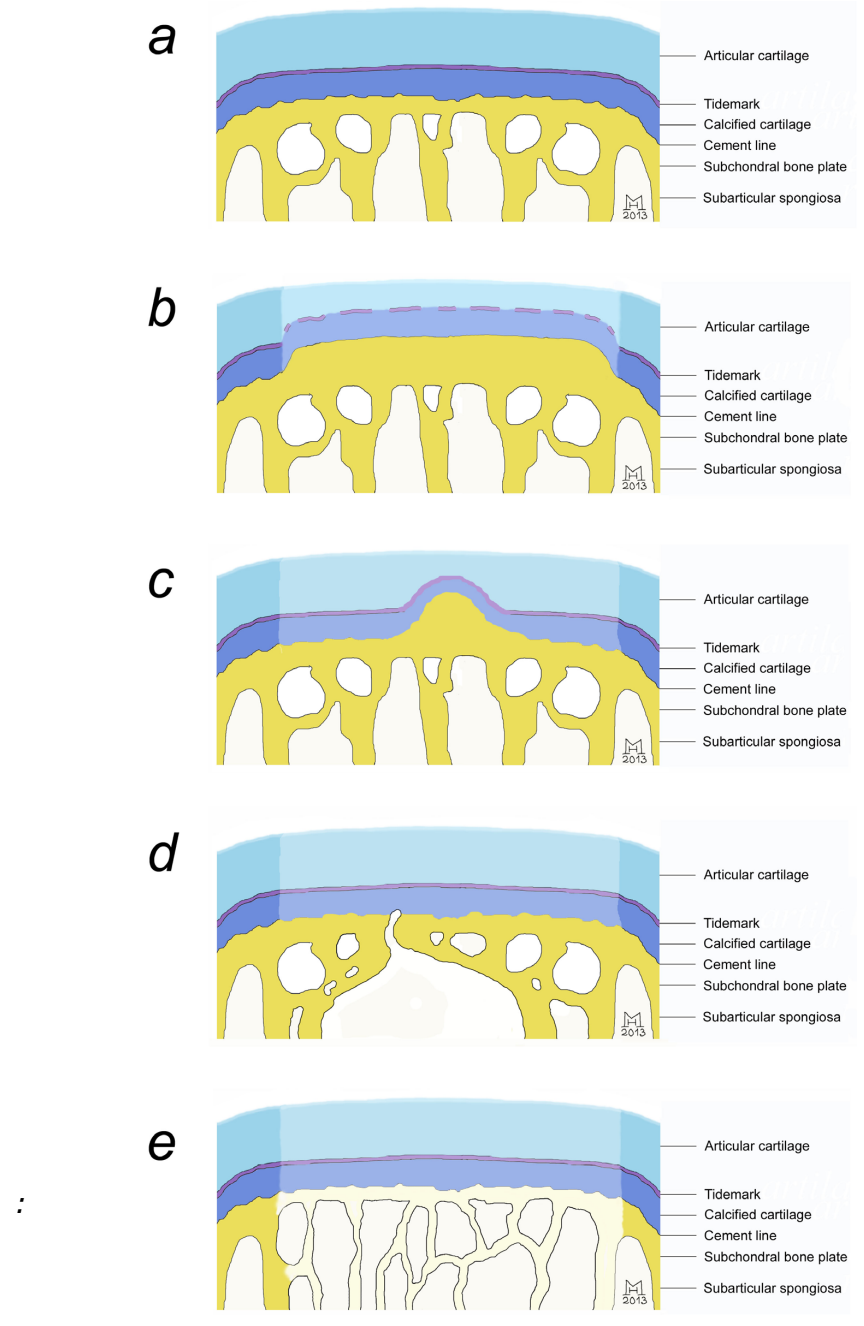

Figure 15. Illustration of subchondral bone alterations. a) normal joint b) progressively upward advance of the subchondral bone plate, leading to thinner articular cartilage c) formation of focal intralesional osteophytes, seen as newly formed bone apical to the original line d) formation of subchondral bone cysts e) impairment of the subchondral bone architecture, and reduced trabecular thickness and overall decreased bone volume. (145) 
Introduction

In a MRI analysis of patients treated with a combination of hyaluronic acid and ACI, 50\% of the patients do show altered subchondral bone, seen as edema, granulation tissue, cyst formation and sclerosis. (148) It is becoming overwhelming clear that clinical solutions must consider the osteochondral joint as one unit, tightly associated in the repair process. Moreover, it can be hypothesized that alterations of the subchondral bone is the reason for negative clinical outcomes in chronic defects and treatments where microfracture previously been the treatment choice. (149) (150) (97) Figure 15 show some of the alterations that can be seen in the subchondral bone. (145)

\section{Previous studies}

This research group has previously studied and characterized porous scaffolds, of PCL and other materials. (151) (117) (122) (152) (153) (154) (155) (125) (142) (121) PCL is a semicrystalline polyester with slow degradability, why it is suitable for long time cell culture in vitro before implantation. The scaffold will maintain the integrity and mechanical properties throughout the cultivation and the implantation. PCL scaffolds have been fabricated with different techniques, from macro porous 
to double pore architecture. Macro porous PCL scaffold was fabricated by a template technique in previous studies. The template was prepared from polymer microspheres, compressed under high temperature. The porosity of the template can be adjusted with the grade of compression. Melted PCL was then injected into the template, and after microsphere leaching a macro porous scaffold was formed. During culture, chondrocytes efficiently maintained their differentiated phenotype and synthesized cartilage specific ECM matrix. The scaffold maintained the mechanical properties and structure. (156) This PCL scaffold was later implanted in a rabbit model, pre-seeded with allogenic chondrocytes, and showed good cellular and ECM molecule distribution. The scaffold was filled with repair tissue after three months and the scaffold maintained the mechanical integrity after implantation. (152) Macro and micro-porous PCL scaffolds were fabricated with a combined freeze extraction and particle leaching method, used to produce the PCL scaffolds in this thesis. It was shown that PCL scaffolds with hydroxyapatite covering give a hybrid interconnected organic-inorganic structure and enhanced bioactivity. (123) In yet another study it was seen that osteogenic differentiation was higher in pure PCL, with macro and micro-porosity, scaffolds, compared to PCL-Ha or PCL-Bio 
active glass composites. (151) Macro and micro porous PCL scaffold have been coated with hyaluronic acid, for different coating and fabrication conditions. Cell cultures have been carried out, showing chondrocyte proliferation and ECM production. (155) PCL porous scaffolds was prepared with hyaluronic acid and used in a rabbit model to asses chondrogenic differentiation. It was established that mesenchymal stem cells from the bone marrow, arriving through the microfracture, preferred a more hydrophilic and biomimetic environment. (154) There are unpublished studies on the PCL scaffold produced by freeze extraction and particle leaching regarding the effects of hypoxia on chondrocytes, cultivation in bioreactor and cultivated with a co-culture of mesenchymal stem cells and chondrocytes.

\section{A biomedical implant device}

Due to this research group previously reported favorable results for porous PCL scaffolds, this thesis is focused on PCL as scaffolding material. One of the thesis task was to adapt an patented biomedical implant, to animal studies. The patent is called "Fixation device for a macro-porous material for the regeneration of articular cartilage" and has patent number EP201131625 PCT/WO2013/178852. 
The implant device is composed by a synthetic and biocompatible macro-porous material with a subchondral bone fixation unit. The system allows the implantation in chondral defects and consists of three units:

- A macro-porous biocompatible sponge with articular cartilage regeneration properties.

- A subchondral bone anchoring system.

- A part that connects the chondral sponge with the subchondral bone anchoring unit.

In this thesis, PCL is chosen as biomaterial and the sponge will be synthesized by freeze extraction and particle leaching process. The subchondral bone anchoring part is chosen to be a Poly(lactic acid) commercial pin. The original implant is designed for human cartilage with a thickness of approximately $3 \mathrm{~mm}$, which allows a third polymeric part to fix the sponge and the anchodring system. In this thesis the implant is adapted for animal studies with a thin cartilage surface, which does not allow more than a porous sponge in the chondral defect. Therefore, the PCL scaffold and PLLA pin will be fixed together in the synthesizing process.

The implant device permits cartilage regeneration by allowing an anchoring system to the subchondral bone, 
without damaging the porous sponge in the chondral defect (Figure 16). The sponge is made of a porous biomaterial and to avoid damaging the porous structure, a polymeric membrane will be attached to the sponge and the subchondral anchoring part. The polymeric membrane will allow the device to be implanted in a chondral defect without affecting the porous material. The polymeric membrane is originally a ring-shaped perforated PCL membrane, attached in the sponge, and can be reached from the top of the implant to facilitate the implantation. The device will be placed over a microfracture defect, and that is why the PCL ring is perforated. After macrofracture, bMSC will invade the porous sponge and be guided to chondrogenic differentiation. The scaffold will be implanted without previously cell-seeding and only employ the patients own bMSC by the microfracture surgery. It is important that the fixation device allow the flow of stem cells, and let them pass the PCL ring throughout the porous sponge. In this thesis the implant will be modified to allow animal trials, and adapted to a thinner cartilage surface. In animals the articular cartilage is approximately $1 \mathrm{~mm}$ thick, and that is only just about to cover a PCL sponge. Chapter seven will describe the design of the modified implants, and the results after two animal studies. 


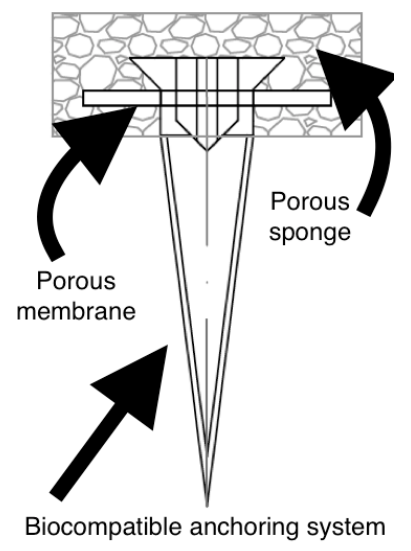

Figure 16. Patent EP201131625 PCT/WO2013/178852, consisting of a porous sponge, a biocompatible anchoring system and a porous membrane.(157)

\section{Experimental techniques to evaluate scaffold efficiency}

It is hard to experimentally evaluate the mechanical efficiency of porous scaffolds in vitro. It is complicated for several reasons: The scaffold itself is not representative for the in vivo situation. In a chondral defect, the scaffold will be filled with cells and ECM molecules and fluid, which together with the scaffold will give a different mechanical response than the scaffold itself. Hence, a porous scaffold tested in water filled or dry conditions do not resemble the actual physiological environment. This is important since water flow have great influence of mechanical outcome, which will be shown in this study. The current 
Introduction

solution is normally to fill the scaffold with chondrocytes and GAGs or collagen fibers to simulate the ECM of cartilage. Bioreactors are used to simulate physiologic conditions. The problem with this is, that cells and ECM molecules are only just representing the initial state of implantation. Once the cells grow and produce ECM the tissue will harden and mechanically change with time. It is also complicated to use living cells for longer experiments as fatigue test. Since tissue constitution change with time, water flow through the tissue is not as simple anymore. There is not enough information how tissue density inside scaffolds influence the mechanical outcome of the implant, and to what parameters this is due. It is not clear what role water permeability plays, and to which extend the hydrogel like tissue inside the scaffolds pore influence the mechanical outcome. This thesis focuses on finding an experimental model that simulates the in vivo situation of an implanted porous scaffold. A micro and macro porous Polycaprolactone, PCL, is designed and characterized and Poly(Vinyl Alcohol) is chosen as tissue mimic. Poly(Vinyl alcohol), PVA, in aqueous solution exhibit the special characteristic of crosslink with repeating cycles of freezing and thawing. (158) By filling the PCL scaffold with the PVA solution, and subject the construct to different numbers of freezing and thawing cycles, interesting features will be 
seen. This gives a usable tool to characterize porous scaffolds in vitro, and predict the mechanical outcome with time from implantation to tissue ingrowth. The experimental model has also been applied on a macro porous scaffold system made of a copolymer of Ethyl Acrylate and Hydroxyethyl Acrylate, with different grade of crosslinking density.

\section{Poly(Vinyl Alcohol)}

Poly(Vinyl Alcohol), PVA, is a water soluble polymer synthetized from polymerization of Vinyl Acetate to Poly (Vinyl Acetate) followed by hydrolysis to Poly(Vinyl Alcohol). The degree of polymerization and hydrolysis affects chemical properties, water solubility and crystallization of PVA. High degree of hydrolysis or higher degree of acetate groups remaining in the polymer affects the thermodynamic properties of PVA. (159) PVA hydrogels can be crosslinked by chemical agents (139), electron beam or $\gamma$-irradiation (160), but these processes leave residual amount of toxic crosslinking agents or form bubbles in the produced hydrogel. One way of avoiding this is to produce PVA hydrogels by freezing and thawing, $\mathrm{f} / \mathrm{t}$, of aqueous PVA solutions. (161) The resulting hydrogel is proposed by other authors as a cartilage 
substitute due to its high equilibrium water content (8090\%), swelling capacities and good mechanical properties. (162) (163) Furthermore the PVA hydrogels are used as medical devices, due to the biocompatibility, chemical resistance and low environmental impact. PVA has been used as soft contact lenses, eye drops, embolization particles and artificial cartilage and meniscus. (164) (165)

\section{PVA hydrogels by freezing and thawing}

Peppas first produced stable hydrogels from aqueous solutions of PVA (Figure 17). He found that the amount of transmitted light through the PVA hydrogels depended on molecular weight, polymer concentration, and time and number of repeating cycles of freezing and thawing. (166) By repeating the amount of $\mathrm{f} / \mathrm{t}$ cycles the stability of the crystallites increases, i.e. degree of crystallinity increased. (167) Other authors have performed X-ray micrographs and have noticed an increase of the crystalline reflection with number of $\mathrm{f} / \mathrm{t}$ cycles. (168) Crystallinity, just as melting temperature, also depend on degree of hydrolysis, since residual acetate groups weaken hydrogen bonding of hydroxyl groups and occupy more space. 


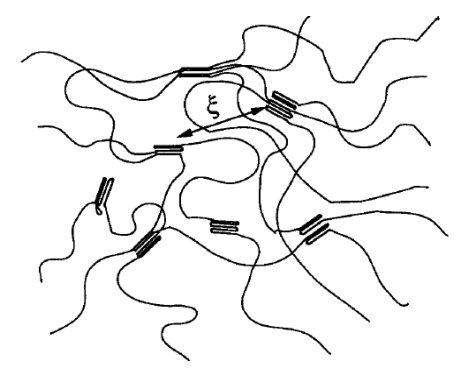

Figure 17. The 3D network of the PVA membranes produced by freezing and thawing by Hickey and Peppas.(167)

Why exactly the hydrogel is formed, is a debated theme and 3 basic theories exist. Some authors believe that network formation is due to hydrogen bonding (169), others claim that it is due to a spinodial decomposition process which results in a polymer rich and a polymer poor phase. (170) (171) Others believe the gelation is due to the organization of network chains into crystallites. (172) (166) There are also theories defending that the phenomena could be connected, (173) explaining the spinodal decomposition as a necessary and at the same time sufficient condition for sol-gel transition. (174) The system would initially be gelled by hydrogen bonding and later network chains would organize into crystallites. Spinodal decomposition results in the development of a polymer rich and a polymer poor region in a fast process. Then, gelation would occur in the polymer rich region, by hydrogen bonding and then crystallite formation. (174) Considering the PVA hydrogel divided in polymer rich and 
polymer poor (ice crystals) phases the repeated cycles of freezing are seen as recrystallization. The polymer rich phase is treated as impurities and will be rejected from the growing ice crystals during freezing. The concentration of polymer in the polymer rich phase will then increase for each cycle of freezing, and the concentration of polymer in the polymer poor phase will decrease and thus create the porous structure. The polymer chains in the polymer rich phase can then hydrogen bond and form a hydrogel. (168) By lowering the concentration of polymer the ice crystals will easier exclude the polymer rich phase and create a larger crystal, and consequently larger pore. PVA hydrogels produced by $\mathrm{f} / \mathrm{t}$ then consists of three phases: a water phase with low polymer concentration (that will form the ice crystals when freezing and form the pores), an amorphous polymer phase where all PVA chains are associated to water molecules, and a crystalline PVA phase that restrain the mobility of the amorphous chains (Figure 18). (168) 


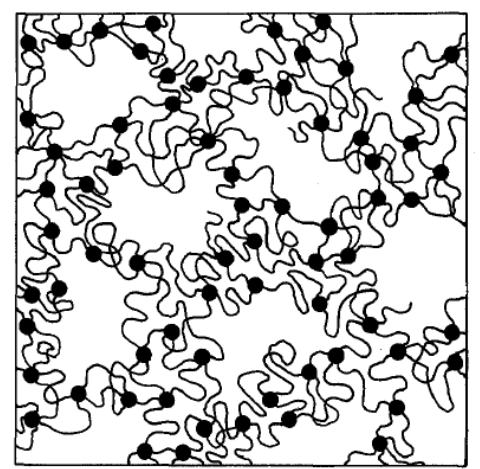

Figure 18. The different phases of PVA hydrogel. Pores are formed by ice crystals and the black circles represent PVA crystallites in a mesh of amorphous PVA chains.(168)

\section{The PVA crystallites}

Differential Scanning Calorimetric analyses have been performed on PVA hydrogels obtained by cycles of $f / t$ (Figure 19 and 20). (175) The hydrogels are not dissolving in polar or nonpolar solvents. For none or two cycles of $f / t$ crystalline melting point was seen around $80{ }^{\circ} \mathrm{C}(176)$ and for twelve cycles $\mathrm{f} / \mathrm{t}$ the crystalline melting point was observed at $220^{\circ} \mathrm{C}$. (175) Glass transition temperature, $\mathrm{T}_{\mathrm{g}}$, was detected as heat peaks in Differential Scanning Calorimetry, DSC, scans or as a decrease in modulus in Dynamic Mechanical Assays, at $45-75^{\circ} \mathrm{C}$ for four to ten cycles of $f / t$, with a strong decrease in the presence of water. (175) (177) (178) Most authors however do not 
Introduction

detect a glass transition, as the samples contain water and the evaporation peak of residual water hides all other possible peaks. Crystalline melting point increases with increasing cycles $\mathrm{f} / \mathrm{t}$ due to increased amount of crystallites. The glass transition temperature increases slightly with increasing cycles $\mathrm{f} / \mathrm{t}$, probably due to that the crystalline formation hinders other hydrogen bonding, and thus strengthen the physical network. (158) (176) (179) (173)

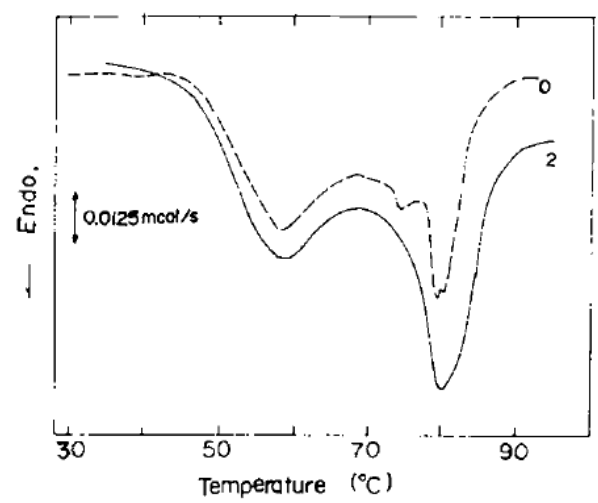

Figure 19. DSC scans of PVA 0 cycles $f / t$ (dashed line) and 2 cycles $f / t$ (solid line). The endothermic peak at $60^{\circ} \mathrm{C}$ is due to the disentanglement of amorphous polymer chains and the peak at $80^{\circ} \mathrm{C}$ is caused by melting of crystallites. (176) 


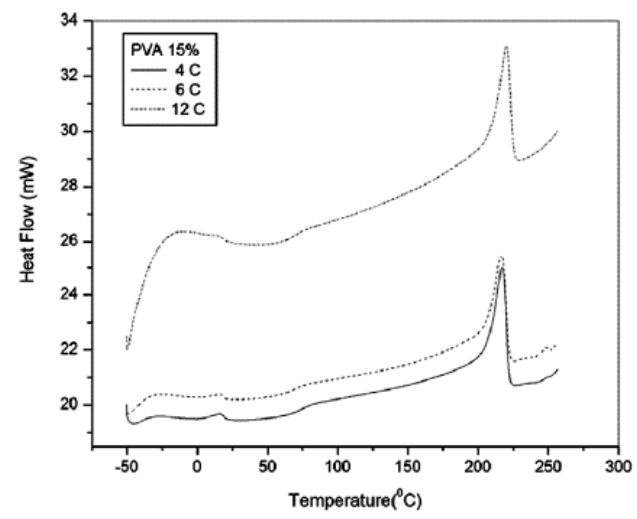

Figure 20. DSC scans of PVA hydrogels with 4,6 and 13 cycles $f / t$. A glass transition temperature is seen from 66 to $70{ }^{\circ} \mathrm{C}$ and crystalline melting point at $217-220^{\circ} \mathrm{C} .(175)$

The crystalline fraction on dry basis was calculated from DSC scans and reached levels as high as $56 \%$ for $10-20 \%$ polymer solution and ten cycles of freezing and thawing. The crystalline fraction of the hydrogel at swollen basis was calculated by dividing the value obtained for crystallinity divided with the swelling ratio, and varied from 0.052 to 0.116. (167) Crystallites in PVA are described as a double layer of molecules held together with hydroxyl bonds and weaker van der Waals forces (Figure 21). A folded chain structure of the PVA leads to small ordered regions, crystallites, in unordered amorphous zones. (180) (181) (182) 


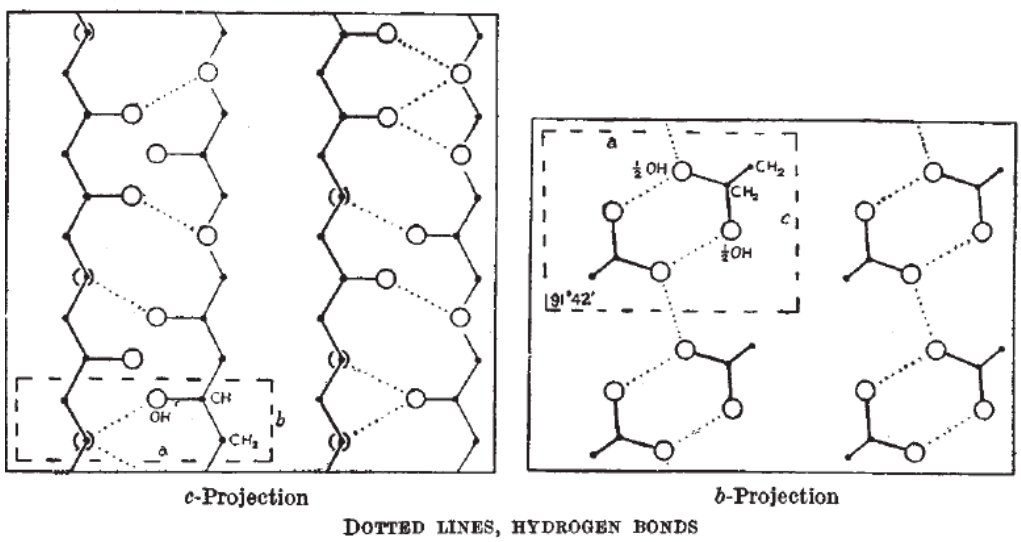

Figure 21. The PVA crystal from X ray crystallography. The dotted lines represent hydrogen bonds. (183)

Hydroxyl groups of PVA are placed randomly in left and right hand position in a layered structure. The double layer of molecules is held together by hydroxyl bonds and weaker van der Waals forces acting between one double layer and another. (183). The grade of hydrolysis influences the crystallization of PVA, since acetyl groups provide increased bulkiness and low water resistance, which prevents the growth and propagation of the PVA crystallites in water environment, slowing down the gelation process. 


\section{Morphology and pore size}

Morphology of PVA hydrogels after different number of $\mathrm{f} / \mathrm{t}$ cycles has been examined by Scanning Electron Microscopy, SEM. After three cycles of freezing and thawing a micro-porous structure was seen with pore size of around $1 \mu \mathrm{m}$. (168) The porous structure of PVA seen in SEM is believed to arise from the cycles of freezing and thawing. Traces of ice crystal growth are left as pores in the gel and observed as a porous structure in SEM. Furthermore the pores are linked linearly together along the normal direction to the freezer plate. It is therefore believed that under freezing, the ice crystals grow along the direction of the temperature gradient. PVA rich phase are segregated around ice crystals and the hydrogel forming process proceeds in the segregated solution phases, forming the continuous porous polymer skeleton. (168) Regarding pore size change with cycles of $\mathrm{f} / \mathrm{t}$, some authors report no change and other authors an increase in pore size with increasing cycles of $f / t$, believed to be caused by crystal growth of ice. Each time the gel is frozen it is a refinement process of the ice crystals. (184) However, the pore size is reported to increase for decreased polymer concentration, 5\% PVA solutions showed pore size of $10 \mu \mathrm{m}$. The PVA is rejected from the 
ice as impurity and for each cycle of freezing and thawing the polymer rich and polymer poor regions increase in their purity, resulting in a pore size increase. (185)

\section{Water in the hydrogel}

DSC studies by other authors show that a considerable amount of water interacts with the polymer chains in PVA hydrogels. Water in hydrogels from $\mathrm{f} / \mathrm{t}$ cycles can be classified in three groups: water that crystallize at the same temperature as normal water (called free water) and water crystallizing at lower temperatures (called restrained or bound water) and water left when resting from the enthalpy of total water (called non freezing water). The non freezing water was shown constant, whereas the bound water and free water were related with the thermal history produced by the cycles of freezing and thawing. Moreover, bound water had a clear relationship with the thermal stability of the gel. Non freezing water in the aqueous solution of PVA were tightly associated with the hydroxyl groups of the polymer. During freezing the molecular motion of PVA cease and at the same time sections of the polymer chain hydrogen bond and serves as crosslinks. According to this, when freezing the aqueous PVA solution, crystallization of water 
occurs and simultaneous hydrogen bonding to neighboring polymer molecules. Ice crystals grow to different sizes, allowing PVA molecules to rearrange and separate from each other. After thawing, the crosslinked molecules can hold a certain amount of water, which structure is different from free water, based on crystallization peaks in the DSC scans. The thermal history affects the size of the ice crystal and also the content of bound water. (186) (187) (188)

\section{Solubility}

Swelling behavior of PVA hydrogels was evaluated after three, five and seven cycles of f/t. (189) In all cases the swelling ratio increased with time until eventually constant volume. The gels with less cycles of $\mathrm{f} / \mathrm{t}$ swelled more than for the gels with higher number of $f / t$ cycles. It was seen an initial rapid swelling and a consequent decrease after 10-30 hours. This is believed to be loss of crystallinity or chain dissolution. After the initial loss of polymer chain the gels maintained stable for over two months. Others investigated the swelling of PVA hydrogels with different grade of hydrolysis and found that the higher degree of hydrolysis, i.e. more residual acetate groups, lower the swelling ratio. The hydrophobic acetate 
groups weaken the intra and inter molecular hydrogen bonding and make the gel swell more. (176)

\section{Mechanical properties}

The hydrogels of PVA made by repeating cycles of $f / t$ have good mechanical properties. The modulus is a function of number of $\mathrm{f} / \mathrm{t}$ cycles, due to the increase density and chain entanglement, (190) (162) (173) and the modulus also increase with polymer concentration. (174) (188) The mechanical properties are dependent on the temperature of the hydrogels. For increasing temperature the modulus decreases, probably due to melting of crystalline regions. The dynamic viscosity was measured for the hydrogels and a decrease in viscosity for increasing cycles of $f / t$ was observed, implicating that the gel is not fully developed after only one cycle. (179) (176)

\section{Polycaprolactone}

Polycaprolactone, PCL, is a semicrystalline biodegradable polyester with a glass transition temperature of $-60{ }^{\circ} \mathrm{C}$ and a melting temperature at $60{ }^{\circ} \mathrm{C}$. At room temperature, or body temperature, the semicristalline structure of PCL results in a rubbery tough material. PCL can be degraded both enzymatically and hydrolytic under physiological 
conditions. By hydrolysis in water or in PBS the PCL reached a weight loss of $20 \%$ in 60 weeks. The enzymatic degradation in Pseudomonas Lipase reached 20\% weight loss after 14 weeks. (191) PCL is subjected to hydrolytic degradation due to the susceptibility of its aliphatic ester linkage to hydrolysis. The five $\mathrm{C}-\mathrm{H}_{2}$ groups make the polymer hydrophobic. Different fabrication methods have been used to fabricate scaffolds of PCL. Solvent casting is used to produce PCL films and the surface properties depend on the solvent used. (192) The film is produced by casting a polymer solution on a cold or heated surface and further removal of the solvent. This method has been used to produce PCL film, (192) blends with PLA (193) and nanocomposite scaffolds. (194) (195) (123) (151) (122) Blends of PCL with Poly(Ethylene Glycol), PEG, have been used to fabricate scaffolds by leaching out the water soluble PEG. (196) The solvent casting method is also used in combination with porogen leaching to create porous scaffolds. A polymer solution is cast onto a porogen bed and as the solvent is evaporated the polymer becomes solid around the porogen particles. The porogen can be leached out of a non solvent to the polymer, creating pores in variable size. Interconnected porous PCL scaffolds have also been fabricated by combining solvent casting, particle leaching and polymer leaching with sodium chloride and 
PEG spheres. The results are highly interconnected three dimensional scaffolds. (197)

Thermally induced phase separation is a common method for producing porous scaffolds. (198) The polymer solution is lowered to induce phase separation which can be liquid-liquid, which creates polymer poor and polymer rich phases. The pores in the scaffold are formed by growth of polymer poor phase. If the temperature is lowered enough to freeze the solvent, a solid-liquid phase separation would occur. The PCL can be dissolved in dioxane and frozen with liquid nitrogen. The frozen solution is then immersed in a non solvent to the polymer and frozen solvent can be removed and leave pores in the scaffold. The polymer itself is not dissolved, since the temperature is lower than the freezing point of the polymer. The polymer then remains frozen during the exchange of solvent and non solvent to the polymer. Since the polymer remains in solid phase it maintains its structure during the particle leaching. The non solvent should therefore have a freezing point lower than that of the polymer solution to be in liquid state during the process. PCL scaffolds are produced by immersing the frozen polymer solution in cold ethanol. Freezing point of dioxane is around $11^{\circ} \mathrm{C}$. Ethanol freezes at $-117^{\circ} \mathrm{C}$ and 
used as non solvent to the PCL. Dioxane is interchanged with the cold ethanol and creating micro pores in the scaffold, from residual frozen solvent. The scaffolds can also be made macro porous by mixing the PCL solution with polymer microspheres before the freezing step. The microspheres are not dissolved by cold ethanol and an intact structure remains. By increasing the temperature of ethanol after freeze extraction the microspheres can be dissolved since they are soluble in the solvent at higher temperatures. The polymer concentration, the solvent or the cooling rate can be changed to tailor morphology and characteristics of the scaffold. (121) (117) (199) (200)

Copolymer networks of Ethyl Acrylate and 2Hydroxyethyl Acrylate with varying crosslinking density

Poly(ethyl Acrylate), PEA, is a hydrophobic biocompatible polymer and random copolymer networks with varying hydrophilic ratios can be made by copolymerizing Ethyl Acrylate with 2-Hydroxylethyl Acrylate. The resulting random copolymer network is a biostable and biocompatible polymer. Biostable scaffolds, just as biodegradable implants, should induce tissue integration and promote cell growth and differentiation. The concept biostable means that the scaffold resists physical and chemical degradation in vivo. Nevertheless, most polymers 
that are classified as biostable degrade after long time in vivo, due to the harsh physiological environment. (201) The biological response of different cell types cultured on PEA supports has been studied. Favourable result is seen both in monolayer and in three dimensions, for human umbilical vein endothelial cells, conjuntival epithelial cells, fibroblasts, chondrocytes and osteoblasts. (202) (202) (203) (204) In particular it has been shown that PEA membranes are able to induce fibronectin fibrillogenesis in absence of cells, influencing cell adhesion, ECM organization and degradation, and cell differentiation (205) (206) (207). In this thesis study, a random copolymer network has been synthesized of ethyl acrylate, EA, and hydroxyethyl acrylate, HEA, weight proportion 90:10, with varying amount of crosslinking agent Ethylene Glycol Dimethacrylate, EGDMA. By varying the amount of crosslinking agent the three dimensional structure and mechanical properties of the scaffold can be tailored. By copolymerizing EA with HEA, a hydrophilic component is incorporated in the polymeric network. A certain grade of hydrophilicity is required for cell-seeding. (208) (209) In a recent study (125), a P(EA-HEA) scaffold has been used for cartilage regeneration in a rabbit model, showing that the scaffold guided cartilaginous tissue growth in vivo. PEA and P(EA-HEA) macroporous scaffolds have been 
proposed for the anchoring ring of the cornea prosthesis (142) 


\section{Chapter 2: Materials and Methods}

\section{PCL Scaffold preparation}

Macro and micro-porous Polycaprolactone scaffolds were prepared by a freeze extraction and particle leaching process. (117) (121) (122) PCL, average molecular weight $80000 \mathrm{Da}, \mathrm{Mw} / \mathrm{Mn}<2$, was obtained from Sigma Aldrich (Spain). Microspheres of Elvacite 2043, a mixture of low weight Poly (Ethyl Methacrylate), PEMA, and Poly(Methyl Methacrylate), PMMA, with diameters ranging from 120 to $200 \mu \mathrm{m}$ were purchased from Lucite International (USA). 1,4 Dioxane from Sigma Aldrich (Spain) was used as solvent for PCL, and ethanol, EtOH, (99\% pure from Scharlab, Spain) was used to dissolve the dioxane and PEMA/PMMA in the freeze extraction and particle leaching process. Poly(Vinyl Alcohol), average Mw 130000 Da, 99+ \% hydrolyzed was purchased from Sigma Aldrich (Spain). All the chemicals were used as received and with no further modification.

Solutions in 1,4 dioxane with different PCL concentrations, 15, 20 and $25 \%$ (w/w) (hereby called PCL 15/20/25\%), were stirred during 24 hours until transparent and homogeneous solutions were obtained. Scaffolds with 
micro and macro porosity were fabricated by mixing the different solutions of PCL with PEMA/PMMA spheres in a weight ratio of 1:1. Each mixture was immediately frozen with liquid nitrogen. After approximately one minute the frozen mixture was immersed in precooled ethanol and kept at $-20^{\circ} \mathrm{C}$ for three days with daily changes of EtOH. To remove the PEMA/PMMA spheres the scaffolds were washed in ethanol at $40^{\circ} \mathrm{C}$ during 8 days, changing the ethanol twice every day until the ethanol did not show any leftover traces of PEMA/PMMA. This was tested by letting some of the ethanol evaporate on a Petridish. When PEMA/PMMA still is present, a white compound is seen on the Petridish. The scaffolds were cut with circular stamps and surgical scalpels of $5 \mathrm{~mm}$ diameter and $2 \mathrm{~mm}$ height the second day of the particle leaching. The dimensions off the sample were measured three times before each mechanical assay to assure correct measurements. Once removed all PEMA/PMMA particles the scaffolds were dried overnight in room temperature and standard pressure. Then, the scaffolds were dried in continuous vacuum during one day, or until constant weight.

The scaffolds were immersed in water before the experiments. The water immersion of the hydrophobic scaffolds was done by a solvent change ethanol to water. 
To make sure that all the pores were filled with water, the scaffolds were put in water and subjected to continuous vacuum extraction. After the vacuum immersion, all samples were kept in water 24 hours before the PVA filling or mechanical testing to assure the water penetrated all pores.

Water immersed and dry PCL scaffolds were tested in Differential Scanning Calorimetry, DSC, to evaluate any plasticizing effect on the samples. Heating scans were performed at $20^{\circ} \mathrm{C} / \mathrm{min}$ in a PYRYS-DSC 8000 equipment (Perkin Elmer) under flowing nitrogen atmosphere between $90^{\circ} \mathrm{C}$ and $90^{\circ} \mathrm{C}$ in $20 \mu \mathrm{L}$ aluminum pans for the dry and water immersed (24 hours) PCL scaffolds. The excess water was absorbed by filter paper immediately before introducing the sample in the equipment.

The morphology of the PCL scaffolds was observed by Scanning Electron Microscope (JEOL JSM-5410, Japan), SEM. The samples were broken in liquid nitrogen to obtain the cross-section of the samples, covered with gold and images were taken at an acceleration voltage of $15 \mathrm{kV}$. 


\section{Hydrogel preparation}

A 10\% aqueous solution of PVA, average molecular weight, Mw, 130000 Da and 99+\% hydrolyzed (Sigma Aldrich, Spain), was prepared by continuously stirring at $90^{\circ} \mathrm{C}$ for 1 hour and then let to cool in room temperature. The solution was poured into $5 \mathrm{~mm}$ diameter wells and frozen for 12 hours in $-20^{\circ} \mathrm{C}$ and then thawed back to room temperature in a high humidity chamber for 8 hours. The freezing and thawing step was repeated one to six times.

The water content in the gels was analyzed and calculated for six samples after 1, 3 and 6 cycles of freezing and thawing and overnight immersed in water. The samples were freeze dried with $-80^{\circ} \mathrm{C}$ condensation and pressure < 100 mbar (Lyoquest, Telstar). The mass before and after freeze drying was measured and the difference was considered to be water. A Thermo Gravimetric Analysis (TGA/StarSystem, Mettler Toledo) to $400^{\circ} \mathrm{C}$ was done to evaluate the resting amount of water in the gel. The mass loss until $180^{\circ} \mathrm{C}$ was considered to be water.

The crystallinity was calculated from Different Scanning Calorimetry, DSC, scans. DSC was performed on freeze dried gels for 0, 1 and 6 cycles of freezing and thawing. Heating scans in a PYRYS-DSC 8000 equipment (Perkin 
Elmer) were performed at $20^{\circ} \mathrm{C} / \mathrm{min}$ between $-80^{\circ} \mathrm{C}$ and $280^{\circ} \mathrm{C}$. The heating scan was performed under flowing nitrogen atmosphere in $20 \mu \mathrm{L}$ aluminum pans. Since the first heating scans revealed residual amounts of water, a second scan to $100{ }^{\circ} \mathrm{C}$ was made, to then lower the temperature to $-80^{\circ} \mathrm{C}$ and repeat the scan to $280^{\circ} \mathrm{C}$.

\section{$\mathrm{PCL} /$ hydrogel construct}

The PVA aqueous solution was introduced into the previously water-immersed PCL scaffolds by vacuum injection. The scaffold and gel constructs were subjected to different number of freezing and thawing cycles, under the same conditions as the pure PVA gels. The effectiveness of the PVA filling was calculated by porosity measurements according to equation 2 and confirmed by cryo-SEM images. The density of the PVA solution was estimated from a 10\% PVA aqueous solution with PVA density of $1.30 \mathrm{~g} / \mathrm{cm}^{3}$. (158) The PCL density was taken from others study, with the value $1.146 \mathrm{~g} / \mathrm{cm}^{3}$. (210) The volume of the PVA was calculated as the difference in mass of the filled and empty scaffold divided with the density of the hydrogel. The volume of the PCL is the weight of the dry scaffold divided by the density of the PCL. 


$$
\begin{gathered}
\phi=\frac{\mathrm{V}_{\text {pores }}}{\mathrm{V}_{\text {total }}}=\frac{\mathrm{V}_{\text {pores }}}{\mathrm{V}_{\text {scaffold }}+\mathrm{V}_{\text {pores }}} \\
\mathrm{V}_{\text {pores }}=\frac{\mathrm{m}_{\text {with PVA }}-\mathrm{m}_{\text {dry }}}{\rho_{\text {PVA solution }}}
\end{gathered}
$$

$$
\mathrm{V}_{\text {scaffold }}=\frac{\mathrm{m}_{\mathrm{dry}}}{\rho_{\text {Polymer }}}
$$

The morphology of the PCL/PVA constructs and the pure PVA hydrogels was observed by Scanning Electron Microscope (JEOL JSM-5410, Japan) equipped with a cryogenic device. The hydrogels were frozen at $-80^{\circ} \mathrm{C}$ and broken to see the cross-section of the samples and then sublimated for 40 minutes. Then the samples were covered with gold and images were taken at an acceleration voltage of $15 \mathrm{kV}$. 


\section{P(EA-HEA) scaffolds}

Scaffold series of $\mathrm{P}$ (EA-HEA) were prepared with a template technique previously reported elsewhere. (211) (212) (213) Templates were synthetized by the use of PMMA microspheres (Colacryl DP 300; Lucite International, UK) with a diameter between 80 and 120 $\mathrm{mm}$. The porogen microspheres were placed in a metal mold and subjected to successive compressions at $150{ }^{\circ} \mathrm{C}$ in a hot plates press (CUMIX TO-250/20), to obtain the template in sheet form of approximately 2 millimeters with a suitable interconnection of porogen particles. Mixtures of $90 \%$ ethyl acrylate, EA (Scharlau 99\%), and $10 \%$ hydroxyethyl acrylate, HEA (Aldrich 96\%), with 0.5 \% Benzoine (Scharlau 98\%) and 1, 3 or $5 \%$ of crosslinking agent Ethylene glycol dimethylacrylate, EGDMA (Aldrich 99\%) was prepared. The monomer solution was poured into the template plates and put into UV Owen 24 hours for radical polymerization. A 24 hours post polymerization in $90^{\circ} \mathrm{C}$ was realized to eliminate residual monomers. Once polymerized the membranes were washed with three changes of acetone (Fluka 99,9\%) for 4 days, 12 hours between each solvent change, in room temperature during agitation to eliminate the PMMA spheres. To obtain the porous three dimensional scaffold a acetone-water 
solvent exchange was performed, slowly to avoid pore collapse. Once obtained the water immersed scaffolds they were left in water during 24 hours to assure no leftover traces of acetone. The P(EA-HEA) membranes were cut into $3 \mathrm{~mm}$ diameter and $2 \mathrm{~mm}$ high scaffolds with circular stamps and chirurgical scalpels. The porosity of the porogen template and the P(EA-HEA) scaffolds (6 replicas of each sample) was calculates using equation 3 where: M is the template weight of the PMMA/scaffold, $\rho$ is the density of the polymer and $\mathrm{t}, \mathrm{w}$ and $\mathrm{l}$ is the height, width and length of the template (214).

$$
\phi=1-\frac{m / \rho}{t * w * l}
$$

The density of the P(EA-HEA) scaffolds was determined through Archimedes principle. The dry sample was weighed in air and immersed in n-octane. A Mettler AE 240 balance with a sensitivity of $0.01 \mathrm{mg}$ with a Mettler ME 33360 accessory was used in the measurements as described in others works (215) (216). The calculated density for P(EA-HEA) is $1,1363 \mathrm{~g} / \mathrm{cm}^{3}$. The value is 1,19 $\mathrm{g} / \mathrm{cm}^{3}$ for the PMMA spheres (Suppliers value). 


\section{P(EA-HEA)/PVA constructs}

PVA aqueous solution was introduced into already water immersed P(EA-HEA) scaffolds by vacuum injection. The scaffolds were subjected to a number of successive freezing and thawing cycles as described for the PVA gels. The effectiveness of filling was calculated with porosity calculations based on weight and dimensions of samples according to equation 2. The density of the PVA solution was estimated from a 10\% PVA aqueous solution with a pure PVA density of $1.30 \mathrm{~g} / \mathrm{cm}^{3}$ (158). The density of $\mathrm{P}($ EA-HEA) was calculated as described above.

The morphology of P(EA-HEA) scaffolds and the P(EAHEA)/PVA construct was observed by Scanning Electron Microscope (JEOL JSM-5410, Japan) equipped with a cryogenic device. The hydrogel filled samples were frozen at $-80^{\circ} \mathrm{C}$ and broken to see the cross-section of the samples and then sublimated for 40 minutes. The empty scaffolds were observed in the same equipment without the cryogenic device. Images were taken at an acceleration voltage of $15 \mathrm{kV}$. 


\section{Unconfined compression}

Unconfined compression tests were performed to the different scaffolds to calculate different mechanical properties. The elastic modulus, the pore collapse and the stress relaxation behavior was examined. The PCL scaffolds were tested in water immersed and dry conditions for different micro porosity, and filled with PVA hydrogel for 1-6 cycles of freezing and thawing. The PVA hydrogel it self was tested for 1-6 cycles of $f / t$. The P(EAHEA) scaffold was tested in immersed and dried conditions for different grade of crosslinking agent and filled with PVA 6 cycles $\mathrm{f} / \mathrm{t}$. Six samples of each group were tested and one way ANOVA statistical test was done $(\mathrm{p}<0.05)$ and unpaired t-test with Welch correction was used to evaluate statistical significant differences between groups. One way ANOVA can compare differences between more than two groups and the t-test is considered a special case of the one way ANOVA and can only compare two groups.

Mechanical characterization of the samples was performed using a Microtest SCM 300095 Universal testing machine (Spain) with a $15 \mathrm{~N}$ load cell. The samples were exposed to unconfined compression cycles to $15 \%$ 
strain at a rate of $1 \mathrm{~mm} / \mathrm{min}$, considered to be within the physiological deformation suffered by natural cartilage (217) (218) (219), in a custom made device that allows the samples to be immersed in water during the assay. The data results of the experiment were used to make a stressstrain representation. The elastic modulus was calculated as the slope at $2 \%$ of deformation, and the maximum stress at $15 \%$ deformation was used to compare between samples. Furthermore, a test of total collapse of the samples was made by using the same equipment and a compression to maximum $15 \mathrm{~N}$, to distinguish the different zones of the pore collapse.

A stress relaxation test was done in the same equipment by unconfined compression for 5, 10 and $15 \%$ of displacement. The sample was allowed to relax at those deformations for two hours (220) (221). A logarithmic stress-time representation was made to obtain the stress relaxation behavior of the samples with time. A linearity test was done by a stress-strain representation and a linear fit between the points for 1, 10, 100 and 1000 seconds of relaxation (222). 


\section{Fatigue tests}

The PCL scaffolds were tested for long-time mechanical behavior by fatigue tests. The scaffolds were tested in dry conditions, immersed in water and filled with PVA gel for 6 cycles of $\mathrm{f} / \mathrm{t}$. The scaffolds were subjected to 100,3000 , 10,000 and 100,000 cycles of $1 \mathrm{~Hz}$ sinusoidal compression of $15 \%$ strain in a Microtest Universal Fatigue machine with a $1500 \mathrm{~N}$ cell. The chosen strain of $15 \%$ ensures that the samples are subjected to a higher compressive fatigue strain than the samples would suffer inside the human body, which would be less than $6 \%$ strain (30). The number of cycles has been chosen to compare short-time with long-time effects inside the scaffolds. The frequency of $1 \mathrm{~Hz}$ is chosen to imitate the frequency of a normal human step (223) After each fatigue time the samples were measured in a Thermo-Mechanical Assay machine (TMA) Seiko TMA/SS6000 (Japan), with two successive programs of loading and unloading to $100 \mathrm{~g}$ with a rate of $10 \mathrm{~g} / \mathrm{min}$ in room temperature. The experiments in the TMA machine were also made for the samples without fatigue, serving as control. The compliance of the samples have been calculated as the inverse slope of the initial slope of the stress-strain curve obtained in the TMA. The compliance was calculated since the experiments were 
made in a force-controlled mode. Six samples of each group were tested and one way ANOVA statistical test was done $(\mathrm{p}<0.05)$ and unpaired t-test with Welch correction was used to evaluate statistical significant differences between groups.

\section{Confined compression}

The PCL scaffolds were tested in confined compression test for water immersed conditions and filled with PVA hydrogel for 6 cycles of $\mathrm{f} / \mathrm{t}$. The PVA hydrogels were tested for 1-6 cycles of $\mathrm{f} / \mathrm{t}$. The confined compression tests were performed in a Microtest SCM 300095 Universal testing machine (Spain) with a custom made device allowing the sample to be in confined mode during the compression. The sample was placed in a cylindrical chamber with impermeable lateral surface, and permeable bottom surface. The mechanical test consisted of a series of successive deformations at a constant speed of $1 \mathrm{~mm} / \mathrm{min}$, followed by a relaxation period of $15 \mathrm{~min}$. Five successive deformations were performed to $2,4,8,12,16$ and $20 \%$ of strain. Equilibrium stress and strain data were measured at the end of the relaxation time for each strain increment (224) (225) (40) (226) (227). The uniaxial aggregate compressive modulus $\left(H_{A}\right)$ was obtained from the linear fit 
of this data. Although the relationship between the aggregate modulus and strain for large strains is not linear (228) for strains up to $20 \%$ the assumption of linear behavior can be used (229) (230). The aggregate modulus is a measure of the compressive resistance of the solid phase at equilibrium.

According to the linear biphasic theory, stress relaxation time dependence for large times can be described by a single-exponential function with a relaxation time, $t$, given by equation 4 (40) (227) (231) (121)

$$
\tau=\frac{h^{2}}{\pi^{2} H_{A} k}
$$

Where $h$ is the sample thickness, $H_{A}$ is the aggregate compressive modulus and $k$ is the hydraulic permeability in the $z$ direction. Therefore, by fitting the stress $v s$. time relaxation data to the single-exponential function, the relaxation time was calculated, and thus, the hydraulic permeability of the sample.

Six samples of each group were tested and one way ANOVA statistical test was done $(\mathrm{p}<0.05)$ and unpaired $\mathrm{t}$ test with Welch correction was used to evaluate statistical significant differences between groups. 


\section{Indentation}

Indentation test were performed at the cartilage explants from the animal trials. For mechanical experiments, the sheep were sacrificed after 4,5 months and 9 months. Once the sheep were euthanized the samples were obtained by dissection of the right knee and exposure of the internal femoral condyle, by osteotomy with an oscillating saw. The left knee was also obtained by dissection and at the same time the internal femoral condyle was obtained, to use as controls. The samples were sent in sterile PBS buffer medium with penicillin/streptomycin $1 \mathrm{ml} / 100 \mathrm{ml}$ PBS with $0.5 \%$ azide. The temperature was controlled to 4 ${ }^{\circ} \mathrm{C}$ from explant before mechanical testing. After the mechanical testing the samples were fixed to be sent to histology examination. The samples were fixed in formalin solution $10 \%$ neutral buffer for 48 hours. Then the samples are washed and saved in PBS + azide. For the indentation test the specimen was cut with oscillating saw to smaller pieces to fit in the experimental instrument. A cylindrical rod of $1.025 \mathrm{~mm}$ diameter with a flat surface was used in Thermo-Mechanical Assay machine (TMA) Seiko TMA/SS6000 (Japan). The experiment was carried out in immersed conditions and $38{ }^{\circ} \mathrm{C}$ under force controlled mode. The compression program was set to 
loading ramp of $125 \mathrm{~g}$ with velocity $10 \mathrm{~g} / \mathrm{min}$ and unloading to $0 \mathrm{~g}$ with the same velocity. In the stressstrain graph plotted from the data obtained, the first strains are neglected until a positive force slope. For the calculations Poisson's coefficient is estimated to 0.4 and the thickness of the cartilage is estimated from posterior histology photos. (42) (152) 
Chapter 3: An in vitro experimental model to predict the mechanical behavior of macroporous scaffolds implanted in articular cartilage

This chapter is based on the published article "An in vitro experimental model to predict the mechanical behavior of macroporous scaffolds implanted in articular cartilage" in the Journal of the Mechanical Behavior of Biomedical Materials Volume 32, Pages 1-352 (April 2014).

This is the first study that describes the experimental cartilage model proposed in this thesis. The model intent to assess the mechanical properties of tissue engineering scaffolds, and predict their performance in vivo during tissue regeneration. The model is developed on a PCL scaffold synthesized with freeze extraction and particle leaching method. The PCL scaffold was made from a solution of $15 \%$ polymer and rate 1:1 with PEMA/PMMA particles. To simulate the growth of tissue inside the scaffold pores, the scaffold is swollen with an aqueous solution of Poly(Vinyl Alcohol) and subjected to repeated number of cycles of freezing and thawing. In this way the Poly(Vinyl Alcohol) becomes a gel whose stiffness increases with the number of freezing and thawing cycles. 
Unconfined compression tests and SEM observations have been done to characterize the scaffolds. The PCL/PVA construct was subjected to 1-6 cycles of $\mathrm{f} / \mathrm{t}$ and mechanical assays was done for each cycle of $f / t$. The pure PVA hydrogels were subjected to 1-6 cycles of $\mathrm{f} / \mathrm{t}$ and tested as controls.

\section{Results and Discussion}

SEM images show that the PCL scaffold shows a double interconnected porosity with macro-pores ranging from $120 \mu \mathrm{m}$ to $200 \mu \mathrm{m}$ approximately. The macro-pores are produced by the porogen leaching process. The micropores are approximately $10 \mu \mathrm{m}$ and result from the extraction of dioxane crystals formed in the freeze extraction process (Figure 3.1a). The microstructure is similar to that obtained in previous studies. (121) (123) (232) (122) (155) Figure 3.1b shows the PCL scaffold filled with the PVA gel after 6 cycles of freezing and thawing. It is clear that the gel enters both the macro and micro-pores. Cryogenic-SEM was used to assess the microstructure of the PVA gels to conserve the structural swelling. Figure 3.1c shows the porous structure of the pure PVA gel, visible only at higher magnifications. 
An in vitro experimental model to predict the mechanical behavior of macroporous scaffolds implanted in articular cartilage

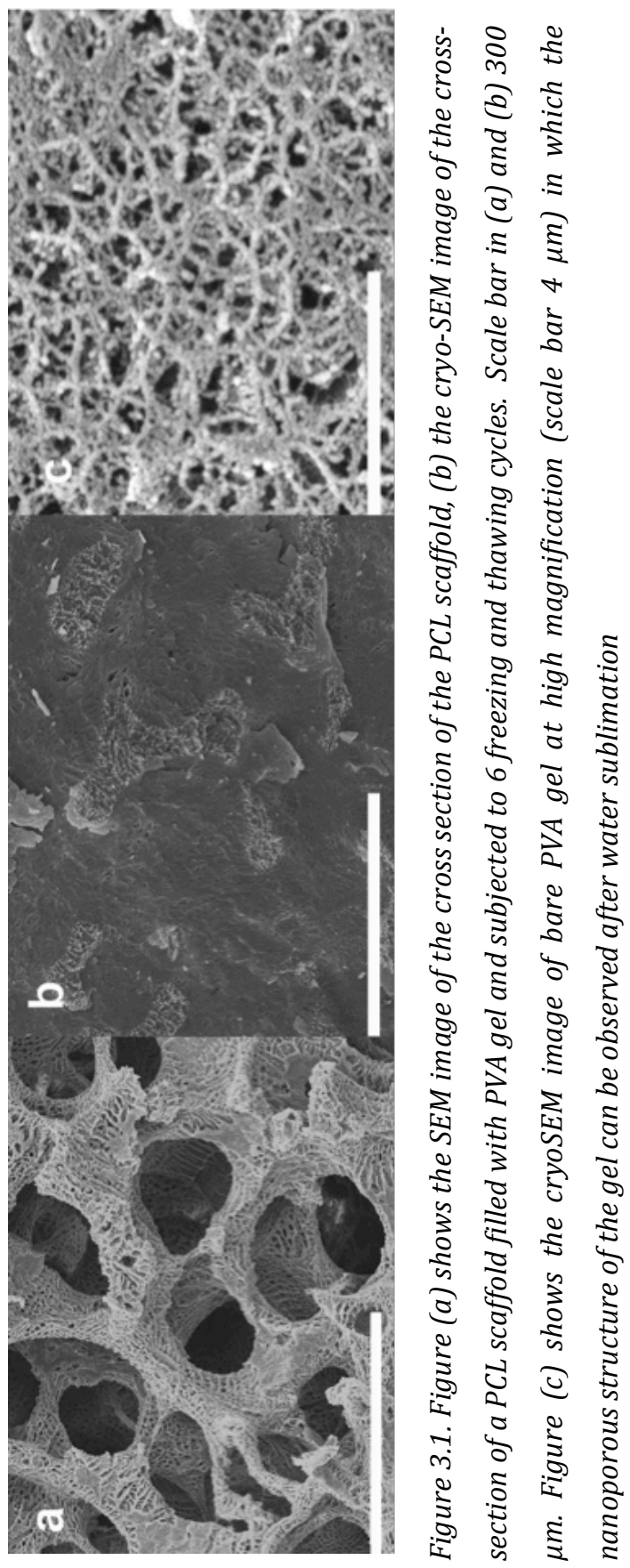


Natural articular cartilage can be modelled as a biphasic structure, with a solid porous permeable phase, the tissue, and a fluid phase. Cartilage tissue consists of extracellular matrix produced by chondrocytes, which organize and maintain the structure of the cartilage by continued reorganization activity. Cartilage is mainly formed by collagen type II fibres and glycosaminoglycans (GAGs), which are responsible for high water absorption capacity. The particular mechanical properties of articular cartilage arise mainly from the interactions of water, electrolytes and the collagen and proteoglycans polymeric matrix. When cartilage is deformed, water flows through the tissue allowing limited deformation and giving a viscoelastic response. Therefore it is the biphasic structure of cartilage that determines its load-bearing capacity. Pressure makes fluid flow through the permeable solid phase. (233) (234) (42) (69)

This study intends to obtain an experimental model that simulates the mechanical behavior of the porous scaffold when cartilaginous tissue grows inside the pores. The mechanical response of a scaffold with pore architecture immersed in water, such as that shown in Figure 3.1a, is expected to be quite different from that of healthy cartilage because the dynamics of the water flow through 
An in vitro experimental model to predict the mechanical behavior of macroporous scaffolds implanted in articular cartilage

the pores is different. Nevertheless, it is expected that the scaffold will be progressively filled with extracellular matrix that is produced by the cells, that are previously seeded or invading the scaffold in vivo. This would show that the mechanical properties of the scaffold/newly formed tissue construct vary in time. (108) (235) (236)

The effect of water diffusion on the stiffness of the scaffold under compression is clearly demonstrated by the dependence of the stress-strain curve on the strain rate. Figure 3.2 shows the stress-strain plots of the PCL/PVA constructs measured at different deformation rates from 0.02 to $1 \mathrm{~mm} / \mathrm{min}$. At high compression rates, the water occupying the pores behaves as an incompressible fluid that resists the compression force, which increases the apparent elastic modulus of the construct. At low compression rates, water is able to escape from the sponge making the sample more compliant and thus the measured apparent elastic modulus decreases. Physiological loading frequencies in the articular cartilage are normally listed between 0,1 and $2 \mathrm{~Hz}$. (223) To both simulate the natural conditions and to assure the effect of water within the sample, $1 \mathrm{~mm} / \mathrm{min}$ rate was chosen. That is a loading rate within the physiological range and sufficient to show the effect of the gel within the sample. 


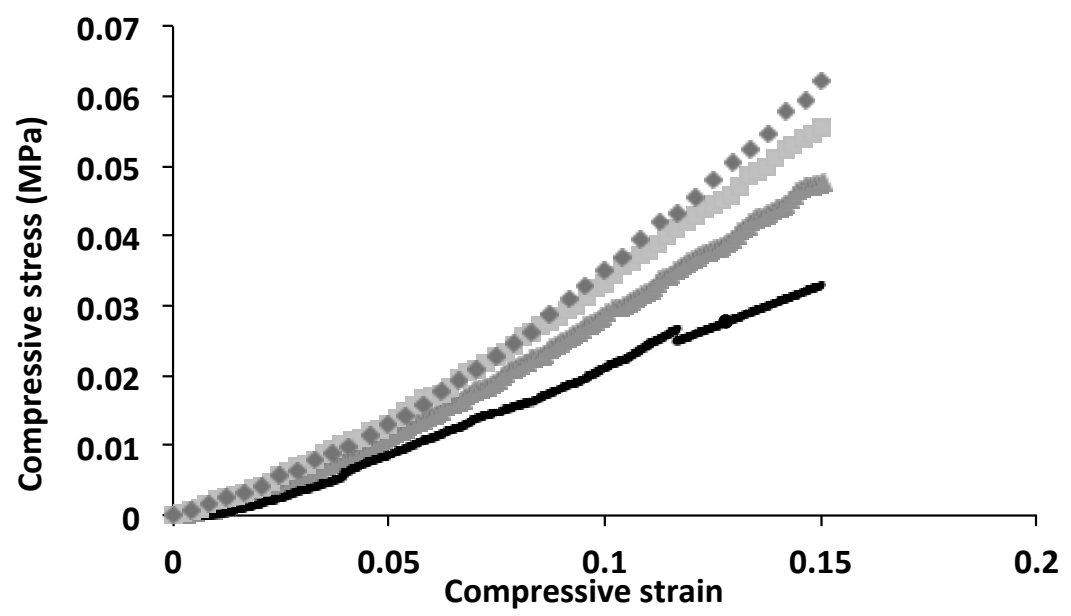

Figure 3.2. Stress-strain curves obtained at varying compression rates in an unconfined compression test of a sample of PCL scaffold filled with PVA gel and subjected to 6 freezing and thawing cycles. The rates used were $1 \mathrm{~mm} / \mathrm{min}$ (grey squares), $0.5 \mathrm{~mm} / \mathrm{min}$ (light grey line), 0.2 $\mathrm{mm} / \mathrm{min}$ (dark grey line) and $0.002 \mathrm{~mm} / \mathrm{min}$ (black line).

Figure 3.3 shows the compression tests performed at a deformation rate of $1 \mathrm{~mm} / \mathrm{min}$ in the water immersed PCL scaffold. Three characteristic regions of scaffold materials can be observed (65): (I) The elastic region corresponding to low strains, (II) the plateau region in which the polymer struts suffer plastic deformation or fracture and pore structure collapses with large deformations at nearly constant stress, and (III) the high slope stress-strain region corresponding to the behavior of the compacted 
An in vitro experimental model to predict the mechanical behavior of macroporous scaffolds implanted in articular cartilage

material. The characteristic regions are labeled in figure 3.3.

The effect of the PVA gel filling the pores is significant in all regions. The plateau is substituted by a continuous increase of stress that shows that collapse of the PCL struts is partly prevented when the pores of the scaffold are filled by the gel. Interestingly enough, the increase in compressive stress in the PCL scaffold/PVA construct cannot be explained by the stiffness of the gel that fills the pores. As shown in Figure 3.3, the elastic modulus of the gel is not very different from that of the PCL scaffold in region II. The clear synergic effect in the construct has to be attributed to the role of the PVA gel to constrain water permeation through the construct after a number of freezing and thawing cycles. Again, incompressibility of liquid water explains the high increase in the compression stress in the construct.

In cartilage application the strain range of interest is limited to $15 \%$, which is considered to be the order of magnitude of physiological deformations suffered by articular cartilage. (217) (218) Values of the elastic modulus were determined in the initial $2 \%$ deformation but due to the significant curvature of the stress-strain plot, the stress at $15 \%$ strain is also presented. 


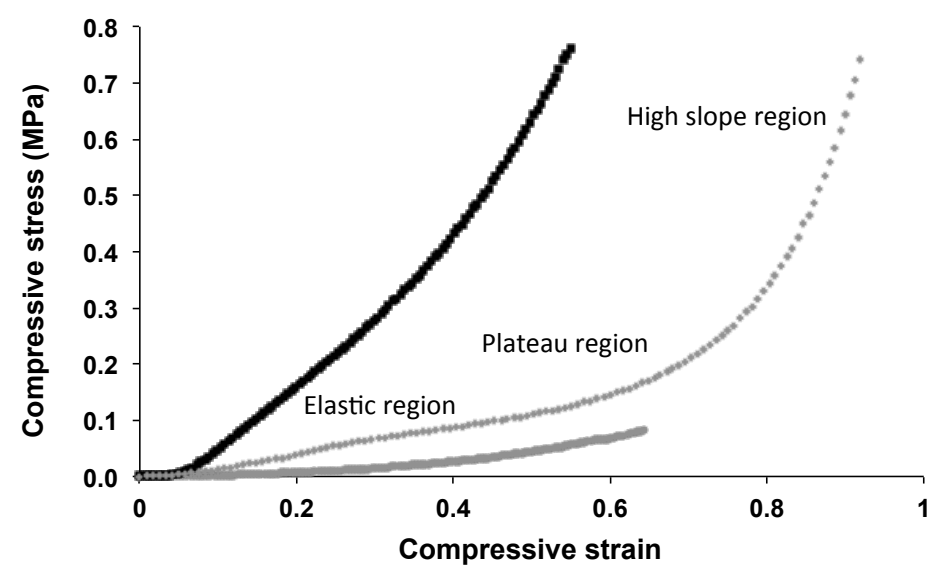

Figure 3.3. Unconfined compression test of the PCL scaffold immersed in liquid water (light grey), PVA gel after 6 freezing and thawing cycles (grey) and the PCL scaffold /PVA construct after 6 freezing and thawing cycles (black).

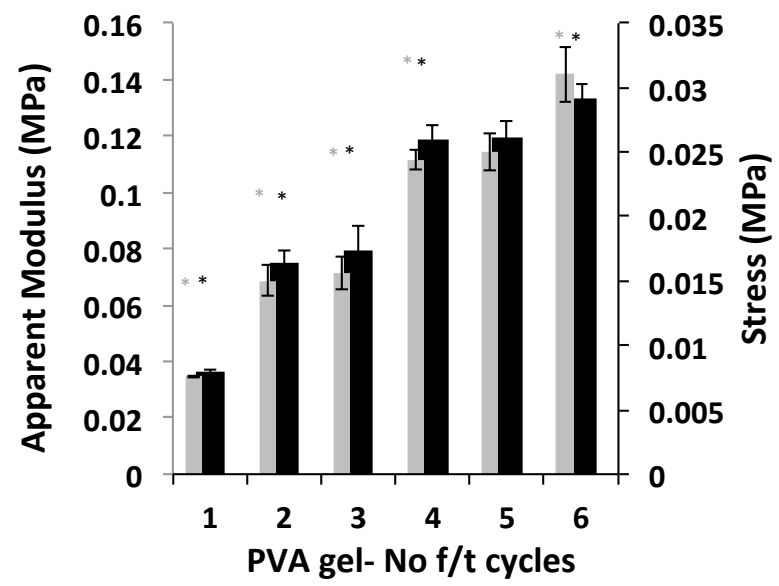

Figure 3.4. The elastic modulus (black) of the PVA gel 1-6 f/t cycles. The stress at 15\% strain is represented in the secondary axis (grey). An ANOVA and t-test analysis was done $(p<0,5)$ for and black (modulus) and grey (max stress) asterisk mark significant difference between samples. 
An in vitro experimental model to predict the mechanical behavior of macroporous scaffolds implanted in articular cartilage

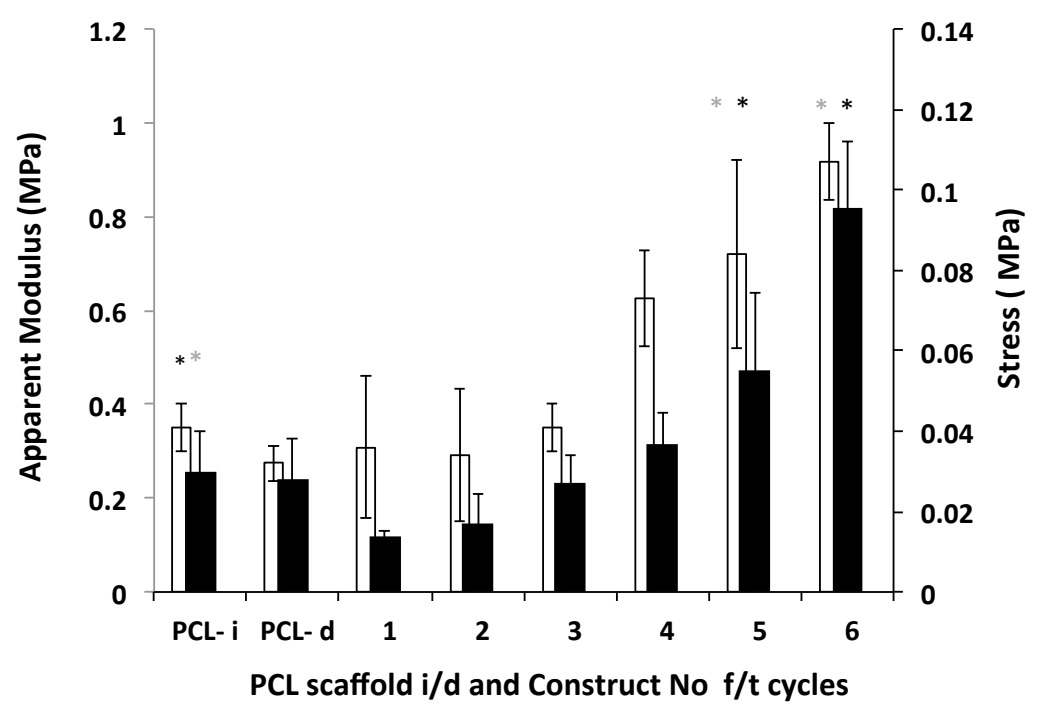

Figure 3.5. The Apparent elastic modulus (black) and stress at 15\% strain (grey) measured in unconfined compression tests in PCL scaffolds (measured in immersion in water-i or dry-d) and the PCL scaffolds/PVA constructs subjected to a different number of successive cycles of freezing and thawing. The asterisk indicates significant differences for (black) modulus and (grey) max stain with respect to dry PCL scaffold according to ANOVA tests with $p<0.05$.

The results obtained in the compression tests of the PVA gel show very low values of one freezing and thawing cycle, around $0.036 \mathrm{MPa}$, but the apparent elastic modulus of the gel increases for every additional cycle. The stiffness increases with the number of freezing and thawing cycles due to the increasing number of crosslinks that will distribute the stress on a higher amount of crystalline regions. This feature has been explained by the increased 
hydrogen bonding between amorphous and or crystalline regions and the densification of regions of amorphous PVA chains. (237) The modulus for the highest crosslinked gel reaches $0.133 \mathrm{MPa}$. The trend for the stress at 15\% strain shows the same behavior with a linear dependence between the stress and the number of freezing and thawing cycles. It is worth noting that the results in Figure 24 do not show any indication of reaching stable values for the stiffness of the gel. As a consequence, we expect that subjecting the gel to further freezing and thawing cycles would lead to an increase of its modulus.

The compression test of the PCL scaffold/PVA construct shows similar behavior (Figure 3.5) as the PVA hydrogels. For one freezing and thawing cycle the modulus of the construct is about $0.12 \mathrm{MPa}$ and for every additional cycle up to 6 cycles the stiffness increases until reaching 0.82 $\mathrm{MPa}$. Interestingly enough, the mean value of the modulus do not depend linearly on the number of freezing and thawing cycles, instead there is an increasing effect on the stiffness with each successive cycle. Furthermore, the mean value of the compression modulus for constructs subjected to 1 to 3 freezing and thawing cycles is even lower than for the PCL scaffolds measured dry or in immersion without PVA. The statistical analyses show that 
An in vitro experimental model to predict the mechanical behavior of macroporous scaffolds implanted in articular cartilage

the differences are not significant between the dry PCL scaffold and the PCL scaffold neither in immersion nor with the construct for up to 3 cycles of freezing and thawing, giving an interesting result. This feature is more explicit in the results of the stress at 15\% strain, shown in Figure 3.5 at the secondary axis. Therefore, filling the hydrophobic scaffold with a soft gel does not change the stiffness of the construct for the first three cycles of freezing and thawing. Not until a densely crosslinked gel is introduced into the scaffold does the stiffness increase. As explained above for higher deformation regions, it seems like the stiffness of the filler itself plays a minor role in the mechanical behavior of the construct, which is mostly determined by the permeability of water in the material. Water diffusion in a very soft gel facilitates the expulsion of water from the scaffold. This would explain why the dry scaffold, the water immersed scaffold and the scaffold/gel construct for up to three cycles of freezing and thawing have very similar mechanical behavior. Note that the water content in the gel filling the scaffold pores is on the order of $90 \%$. According to the literature, PVA gel subjected to an increasing number of freezing and thawing cycles develops a nanoporous structure, in addition to increasing crystallinity. (237) For a higher number of freezing and thawing cycles, in spite of increasing 
nanoporosity, permeability studies suggest a complex relationship between crystallinity, pore interconnectivity, permeability and pore tortuosity that results in a decrease in permeability with the number of cycles. (140)

This singularity explains that in the low deformation regime, the stiffness of the PCL scaffold/PVA construct increases for every cycle of freezing and thawing much more than the stiffness of the PVA gel itself. This behavior is also relevant for the application of macroporous scaffolds in cartilage engineering strategies. The development of newly formed tissue inside the pores could allow the implanted scaffold to reach mechanical properties close to that of the healthy articular cartilage, even if the regenerated tissue is softer than the healthy tissue. This is important for tissue regeneration since it implies that the biomechanical environment of cells hosted in the scaffold pores could be adequate in shorter times than those required for the organization of extracellular matrix with the structure of hyaline cartilage. In fact, it is possible that the extracellular matrix produced within the macro pores of a scaffold do not organize like hyaline cartilage, despite of the fact that chondrocyte markers are expressed. 
An in vitro experimental model to predict the mechanical behavior of macroporous scaffolds implanted in articular cartilage

To emphasize the synergic effect of the scaffold and the hydrogel filling the pores, Table 3.1 shows the values of the apparent elastic modulus in the elastic regime, which can be compared with those of articular cartilage in human or animal models. In previous work authors have conducted indentation studies of the articular cartilage of rabbits. (152) Values of the modulus ranging between 0.26 and $0.59 \mathrm{MPa}$ were measured and the stress for $15 \%$ strain was between 0.08 and $0.3 \mathrm{MPa}$. This shows that the required modulus can be reached with a quite soft hydrogel filling the pores of the scaffold. This is due to the low water permeability of the gel. 


\begin{tabular}{|c|c|c|}
\hline $\begin{array}{c}\text { Sample } \\
\text { PVA 6 f/t cycles }\end{array}$ & $\begin{array}{c}\text { Elastic Modulus } \\
(\mathrm{MPa})\end{array}$ & $\begin{array}{c}\text { Stress at 15\% strain } \\
(\mathrm{MPa})\end{array}$ \\
\hline PCL dry scaffold & $0.13 \pm 0.005$ & $0.032 \pm 0.002$ \\
\hline $\begin{array}{c}\text { PCL/PVA construct 6 } \\
\text { f/t cycles }\end{array}$ & $0.82 \pm 0.14$ & $0.11 \pm 0.009$ \\
\hline $\begin{array}{c}\text { Articular cartilage } \\
\text { (rabbit mode) }\end{array}$ & $0.41 \pm 0.12$ & $0.08-0.003$ \\
\hline
\end{tabular}

Table 3.1. The values of the apparent modulus and the maximum stress for the PCL scaffold, the PVA gel and the scaffold/gel construct. Values for articular cartilage, previously reported, are shown for comparison. (152)

The results obtained with the experimental model proposed in this study show that testing a scaffold to predict its performance during tissue regeneration requires a precise simulation of the contribution of newly formed tissue and of the behavior of the scaffold/tissue construct. This experimental model could be used to obtain a realistic prediction of the performance of a given macro porous scaffold after a number of deformationrecovery cycles as is experienced in vivo during tissue regeneration. Long-term fatigue testing of unfilled scaffolds could significantly underestimate the 
An in vitro experimental model to predict the mechanical behavior of macroporous scaffolds implanted in articular cartilage

performance of the material. Mechanical performance of the scaffold while it is resorbed in vivo could be adequately analyzed as well using hydrolytic or enzymatic media during fatigue testing.

\section{Conclusions}

An experimental model has been proposed to test the mechanical behavior of tissue engineering scaffolds. Newly formed tissue inside the scaffold during the regeneration process is simulated by a PVA hydrogel subjected to a series of consecutive cycles of freezing and thawing. The apparent elastic modulus of the PVA gel increases with the number of freezing and thawing cycles. A PCL scaffold with a pore architecture consisting of spherical interconnected macro pores with micro-pore walls could be efficiently filled with PVA. The stiffness of the scaffold/hydrogel construct increases when subjected to repeated freezing and thawing cycles. Interesting enough, after three cycles $\mathrm{f} / \mathrm{t}$ the elastic modulus of the construct is much higher than that of either of its components. This result is attributed to the effect of the PVA gel hindering water permeation through the scaffold when it is subjected to compression loading. This mechanical behavior is quite similar to that of articular cartilage and could be used to 
predict the performance of scaffolds during degradation or during long-term fatigue testing.

The biphasic theory addresses natural articular cartilage two different constituents, a fluid phase and a solid phase. This theory is also applicable on PVA hydrogels. In cartilage the solid phase is a porous structure of mainly collagen fibres and proteoglycans. The fluid is the interstitial fluid within the porous structure. Articular cartilage is to 70-80 \% made up of water. PVA hydrogels are to $90 \%$ water. The PVA hydrogels constitutes of polymer chains that forms a porous structure where the water can pass through. The mechanical properties of cartilage are highly dependent on the negatively charged GAGs and the water swelling capacity. PVA hydrogels swell readily and its swelling capacity is strictly dependent on its polymer content and number of freezing and thawing cycles. The water is retained within the polymer network. (238) The mechanical properties are to a large extent, in both articular cartilage and PVA hydrogels, dependent on the fluid phase and the permeability of the porous structure. In this study it is clear that the mechanical strength of the hydrogel increases with the number of freezing and thawing cycles. Other authors compare and suggest PVA hydrogels as a substitute for natural articular 
An in vitro experimental model to predict the mechanical behavior of macroporous scaffolds implanted in articular cartilage

cartilage. ESEM images show that both cartilage and PVA hydrogels are porous structures with small holes. (196) In this work we show that it is possible to obtain a scaffold/gel construct with mechanical properties similar to that of the growing matrix of articular cartilage. The stiffness of the PVA hydrogel can easily be tailored by adjusting the number of freezing and thawing cycles. In vivo the stiffness of the scaffold/cell construct will increase with the synthesized new matrix. This is very similar to the in vitro model we have developed with a PCL scaffold and a PVA hydrogel filling the pores. 


\section{Chapter 4: Prediction of the in vivo mechanical behavior of biointegrable acrylic macroporous scaffolds}

This chapter is based on the manuscript "Prediction of the "in vivo" mechanical behaviour of biointegrable acrylic macroporous scaffolds" accepted for publication in Materials Science and Engineering C June 2015.

This study examines a biocompatible scaffold series of random copolymer networks P(EA-HEA) made of Ethyl Acrylate, EA, and 2-Hydroxyl Ethyl Acrylate, HEA. The P(EA-HEA) scaffold has been synthesized with varying crosslinking density and filled with a Poly(Vinyl Alcohol), PVA, to mimic the growing cartilaginous tissue during tissue repair. In cartilage regeneration the scaffold needs to have sufficient mechanical properties to sustain the compression loading in the joint and, at the same time, transmit the stress to the cells for chondrogenic differentiation. This study proposes an experimental model for measuring the mechanical properties of the scaffold in a cartilage defect. The P(EA-HEA) scaffolds are filled with an aqueous solution of Poly(Vinyl Alcohol) and subjected to repeating cycles of freezing and thawing to crosslink the hydrogel inside the scaffolds pores. The 
Prediction of the in vivo mechanical behavior of biointegrable acrylic macroporous scaffolds

mechanical properties of the scaffold/hydrogel construct are measured and the morphology before and after the mechanical tests are evaluated.

\section{Results and Discussion}

The porosity of the porogen template and the P(EA-HEA) scaffolds was calculated by equation 3 . The results from the porosity calculations are listed in table 4.1. The calculated density for P(EA-HEA) is $1.136 \mathrm{~g} / \mathrm{cm}^{3}$. Porosities for scaffolds filled with PVA are all close to 0 and not shown. In table 4.1 it can be seen that the porosity is increasing slightly with increasing crosslinking density. The porosity of the PMMA template is $9 \pm 5$ which gives P(EA-HEA) scaffolds a theoretical porosity of $90 \%$. The final porosity is slightly inferior, showing a certain contraction of the scaffold during template extraction. Materials with higher crosslinking density are closer to this value. 


\begin{tabular}{|l|l|}
\hline Material & $\mathrm{f}(\%)$ \\
\hline P(EA-HEA) 1\% & $78 \pm 4$ \\
\hline P(EA-HEA) 3\% & $83 \pm 3$ \\
\hline P(EA-HEA) 5\% & $87 \pm 4$ \\
\hline PMMA template & $9 \pm 5$ \\
\hline
\end{tabular}

Table 4.1. The porosity of the porogen template and the P(EA-HEA) scaffolds without PVA hydrogel.

All the P(EA-HEA) scaffolds (Figure 4.1a-c) present an interconnected structure with spherical pores before compression tests. It is also possible to observe that the scaffold pores are more open and interconnected, with thinner trabeculare, for higher crosslinking density. The pore size was estimated from 15-20 pores in three photos for each sample. The P(EA-HEA) 1\% scaffolds show a pore size of $15 \div 50 \mu \mathrm{m}$, the P(EA-HEA) $3 \%$ scaffolds between $20 \div 60 \mu \mathrm{m}$ and the P(EA-HEA) 5\% scaffolds between $20 \div 65 \mu \mathrm{m}$. It is hence seen a small increase in pore size with increasing crosslinking density. This phenomenon is produced during the solvent exchange process from acetone to water in the scaffold synthesis. The material with higher crosslinking density absorb less acetone which maintains the material rigid during the solvent 
Prediction of the in vivo mechanical behavior of biointegrable acrylic macroporous scaffolds

exchange, and the result is an interconnected and open pore structure. The scaffolds with smaller crosslinking density can absorb greater amount of acetone and soften during the solvent exchange, which will close the pores. Porosity values (Table 4.1) reflect this fact. After compression tests all materials show a slight morphological change at the scaffold surface (Figure $4.1 \mathrm{~d}$ f). 


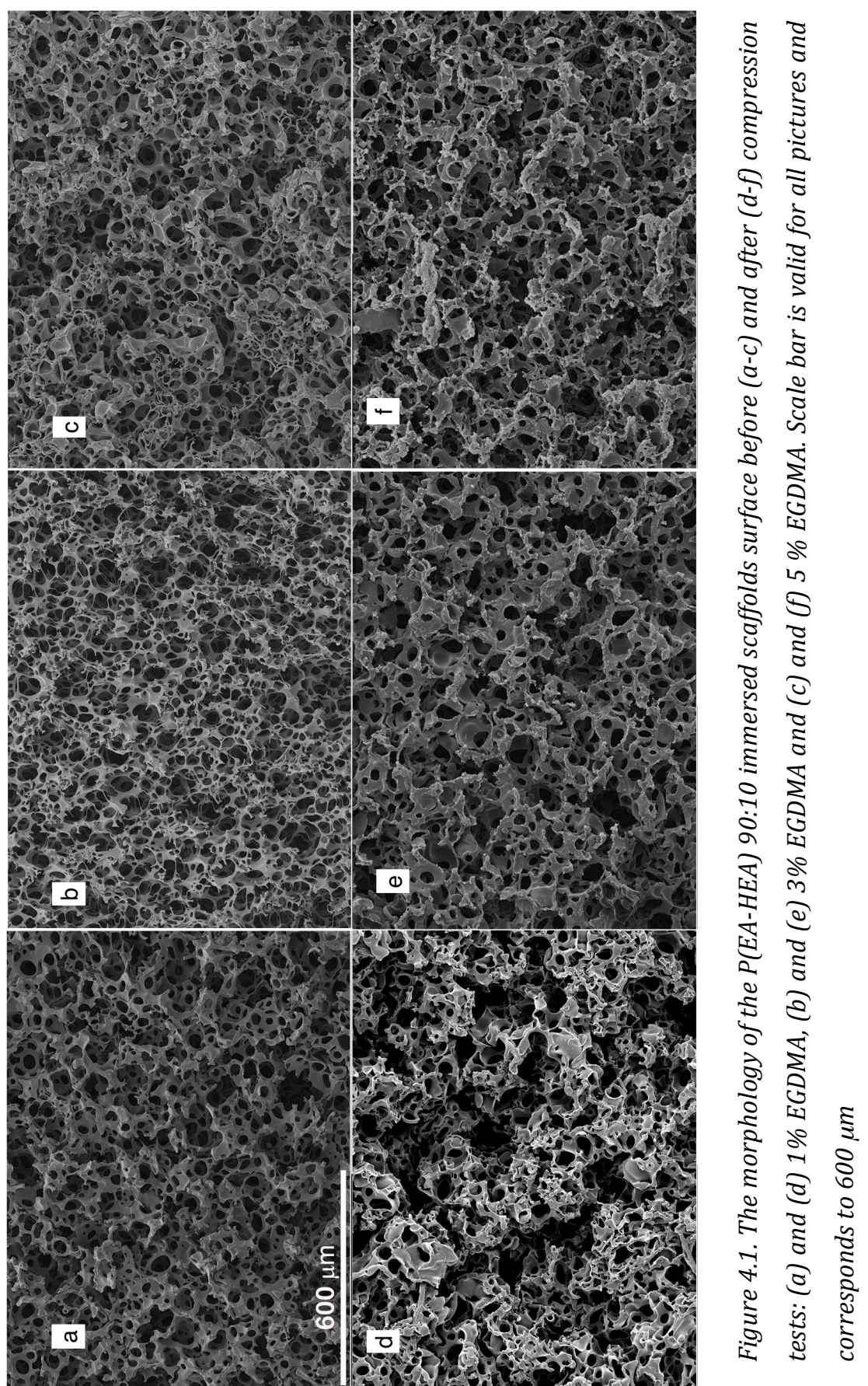


Prediction of the in vivo mechanical behavior of biointegrable acrylic macroporous scaffolds

The influence of crosslinking density on the mechanical properties of the porous scaffold has been assessed by comparing the scaffold with and without water in the pores. It is worth noticing that the P(EA-HEA) scaffold in this study is hydrophilic. The effect of water on the P(EAHEA) scaffolds can only really be evaluated by testing the scaffolds without water in the macro pores, but when the scaffold has absorbed water into the polymer. In this way, water in the macro pores is the only difference between water-filled and water-absorbed samples. Consequently, the scaffolds were immersed in water, and then the water in the macro pores were removed by absorption on filter paper. Both the modulus for the P(EA-HEA) scaffolds immersed in water and for the scaffolds with empty pores increases with increasing crosslinking density (Figure $4.2 \mathrm{a}-\mathrm{c})$. On the other hand, the scaffolds with empty pores (Figure 4.2a-c) show mechanical properties inferior to the water-immersed samples. This indicates that it is foremost water movement inside the scaffold pores that determines the mechanical properties of the scaffold.

Another important observation is that the relationship between the elastic modulus of the water absorbed samples and the water filled samples, for different crosslinking density. For increasing crosslinking density, 
1\%-3\%-5\% EGDMA, the modulus for the water absorbed samples is approximately: $0.05-0.1$ - $0.3 \mathrm{MPa}$ respectively. For the water immersed samples the same scaffolds have modulus of approximately: $0.35-0.55-1 \mathrm{MPa}$. Consequently, the increase is not in the same order for the different samples. P(EA-HEA) 1\% increase the modulus 7 times by having water in the pores, P(EA-HEA) $3 \%$ by 5 and P(EA-HEA) 5\% increases the modulus 3 times. This means that the stiffness of the proper polymer network influence the effect of water on the mechanical properties. As the polymer network becomes more crosslinked, and consequently stiffer, the effect of water filling is less important for the mechanical outcome. On the other hand, there is also a difference in porosity and pore-size between the samples. The decrease in porosity increases tortuosity of the water flowing through the scaffold during compression. This makes the water passage more difficult and hence, the compressive modulus increases. 
Prediction of the in vivo mechanical behavior of biointegrable acrylic macroporous scaffolds

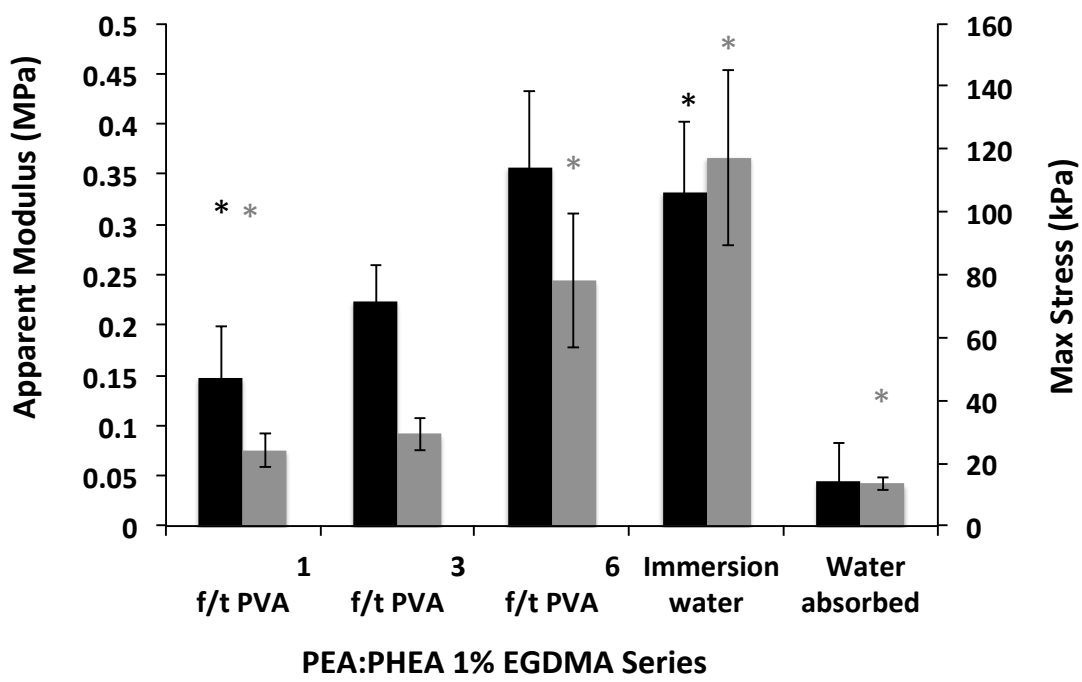

4.2a)

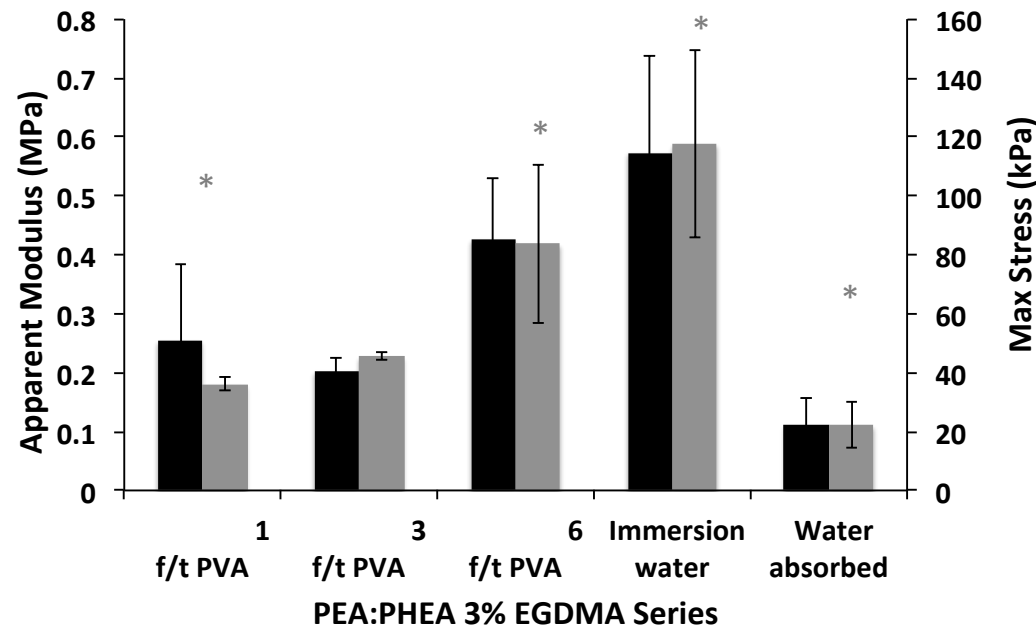

$4.2 b)$ 


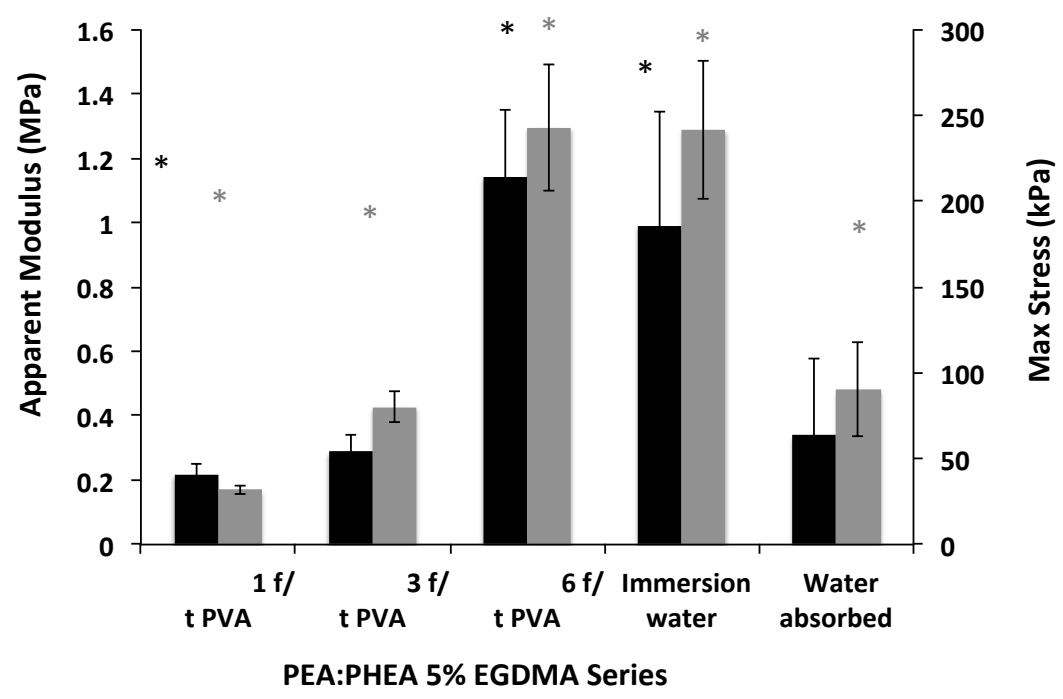

4.2c)

Figure 4.2. The elastic modulus (MPa) and stress for 15\% strain ( $\mathrm{kPa}$ ) in the unconfined compression tests for the P(EA-HEA) series, water absorbed, water immersed and filled with PVA hydrogel for 1, 3 and 6 cycles f/t: (a) P(EA-HEA) 1\%, (b) P(EA-HEA) 3\% and (c) P(EA-HEA) 5\%. Black columns represent modulus and grey stress for 15\% strain. ANOVA statistical analysis and unpaired t-test $(p<0,05)$ were made between groups and significant difference is marked with an asterisk (black between modulus and grey between stress for 15\% strain) in comparison with 1 cycle f/t.

Unmistakably, the capacity of reaching realistic conclusions about the in vivo behavior of the scaffolds is limited with these measurements. The scaffold, when implanted in a chondral defect, will be filled progressively by cells and ECM molecules that form a dense tissue. The 
Prediction of the in vivo mechanical behavior of biointegrable acrylic macroporous scaffolds

PVA hydrogel inside the pores of the scaffold therefore gives a realistic simulation of the in vivo situation. By subjecting the scaffold/hydrogel construct to repeated number of freezing and thawing cycles the PVA becomes stiffer and can be compared to repair tissue. The PVA stiffness can be tailored from an almost viscous solution to a hard hydrogel, simulating the growing tissue inside the scaffold pores. In figure 4.3 it can be seen that the mechanical properties of the pure PVA gels increase with the number of freezing and thawing cycles.

The water content of the pure PVA hydrogels after $6 \mathrm{f} / \mathrm{t}$ cycles was measured as mass loss after freeze drying followed by TGA analysis. The water content after freeze drying was calculated to $86.7 \%$. TGA analysis show that the freeze dried samples still contained $4.1 \%$ water. Hence, total amount of water in the initial samples were $87.2 \%$. 


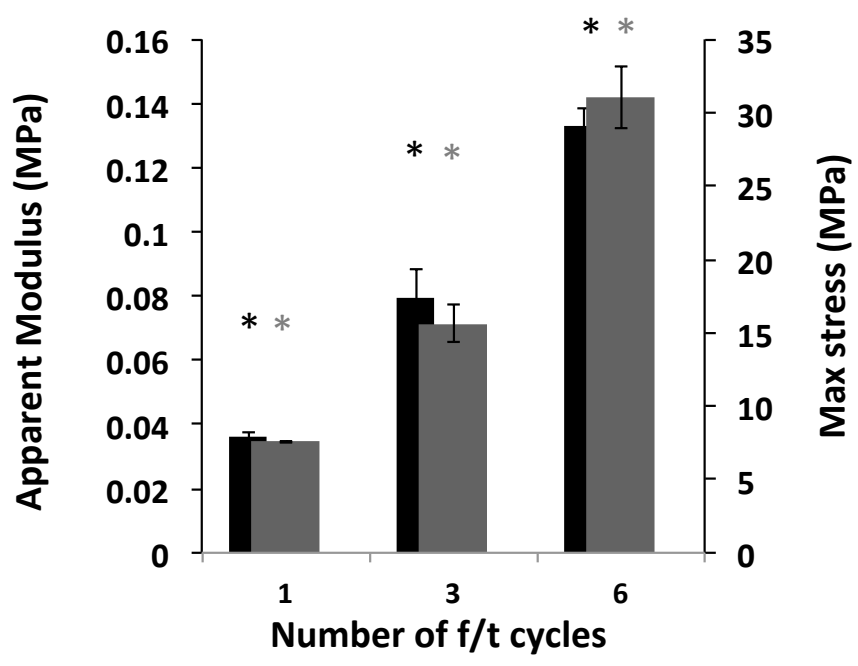

Figure 4.3. PVA gel subjected to 1, 3 and 6 cycles of freezing and thawing in unconfined compression tests in immersed conditions. Black columns represent modulus (MPa) and grey stress for 15\% strain ( $\mathrm{kPa}$ ). ANOVA statistical analysis and unpaired t-test $(p<0,05)$ were made between groups and significant difference is marked with an asterisk (black between modulus and grey between stress for $15 \%$ strain) in comparison with 1 cycle $\mathrm{f} / \mathrm{t}$.

The crystallinity of the pure PVA gels after 0,1 and $6 \mathrm{f} / \mathrm{t}$ cycles was calculated from DSC scans as melting enthalpy divided by $\Delta H_{m}{ }^{\circ}$ for a pure crystalline PVA sample, $150 \mathrm{~J} / \mathrm{g}$ (238). Results are shown in table 4.2. The crystallinity varied from $46 \%$ to $54 \%$ from solution to $6 \mathrm{f} / \mathrm{t}$ cycles. This is coherent with others results, that report crystallinity values of $56 \%$ in hydrogels after 10 cycle $\mathrm{f} / \mathrm{t}$ (167). 
Prediction of the in vivo mechanical behavior of biointegrable acrylic macroporous scaffolds

When the PVA solution is introduced into P(EA-HEA) 1\% scaffolds and subjected to 6 cycles of $\mathrm{f} / \mathrm{t}$ (Figure 4.4a) it can be seen that the hydrogel enters all the scaffolds pores. SEM images of the cross-sektion of the scaffold/hydrogel construct after compression tests (Figure $4.4 \mathrm{~b}$ ) show a slighly different morphology, with a dense gel that seems affected by compression. SEM images of pure PVA hydrogel after 1,3 and 6 cycles $\mathrm{f} / \mathrm{t}$ show a porous structure with a disperse pore size $(0.5-2 \mu \mathrm{m})$ for all cycles of $\mathrm{f} / \mathrm{t}$. Figure $4 \mathrm{c}$ shows the hydrogel after 6 cycles $\mathrm{f} / \mathrm{t}$, which is representative for all cycles $\mathrm{f} / \mathrm{t}$.

\begin{tabular}{|l|l|}
\hline Sample & Crystallinity (\%) \\
\hline PVA 0 cycles f/t & 46.4 \\
\hline PVA 1 cycles f/t & 48.6 \\
\hline PVA 6 cycles f/t & 54.7 \\
\hline
\end{tabular}

Table 4.2. The crystallinity of the PVA solution and hydrogels calculated from DSC scans. 

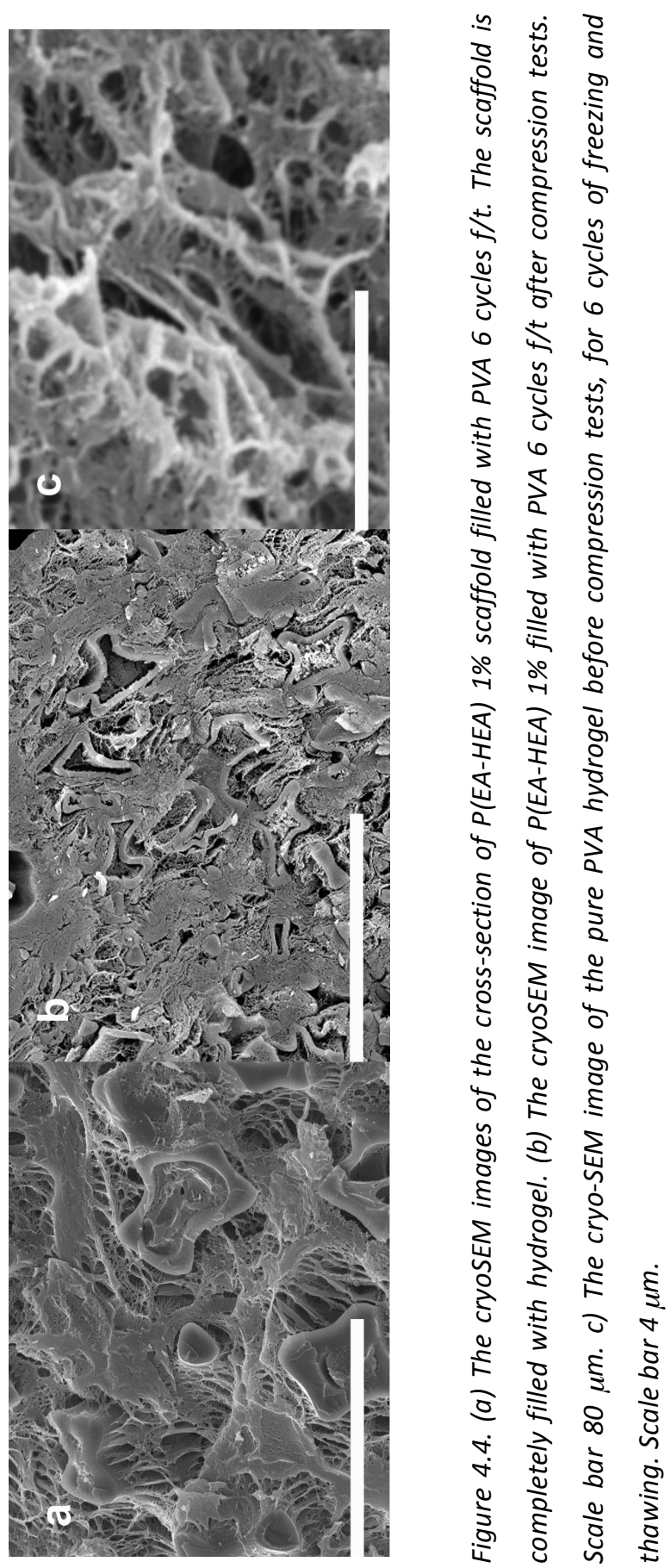
Prediction of the in vivo mechanical behavior of biointegrable acrylic macroporous scaffolds

In this study the macro porous $\mathrm{P}$ (EA-HEA) scaffolds with different crosslinking density were filled with an aqueous solution of Poly(Vinyl Alcohol) and subjected to 1, 3 and 6 cycles of freezing and thawing. Porosity results (Equation 2) and SEM images (Figure 4.4) show that the PVA enters all pores in the scaffold. The mechanical properties of the pure PVA hydrogels increase with increasing number of $\mathrm{f} / \mathrm{t}$ cycles (Figure 4.3), and the same pattern can be seen for the hydrogel filled scaffolds (Figure 4.2), albeit not always significant differences are found. In all P(EA-HEA) scaffolds filled with PVA the elastic modulus increases with respect to the pure PVA gel. Interestingly enough, the mechanical properties for the PVA filled scaffolds decrease compared to the scaffolds immersed in water. When a soft hydrogel is inside the scaffold pores, the water flow is facilitated and expulsed more easily why also the mechanical properties decrease. Only for samples with 6 cycles of $\mathrm{f} / \mathrm{t}$ the mechanical properties are as for the water immersed scaffolds. Comparing the values to articular cartilage in a rabbit model, $0.41 \pm 0.12$, MPa (152) and human, $0.581 \pm 0.168 \mathrm{MPa}(239)$, it can be seen that the P(EA-HEA) 1\% reaches values of the rabbit model for immersed samples. P(EA-HEA) 1\% filled with PVA 6 cycles $\mathrm{f} / \mathrm{t}$ and $\mathrm{P}(\mathrm{EA}-\mathrm{HEA}) 3 \%$ and $5 \%$ immersed in water or filled 
with PVA 6 cycles $\mathrm{f} / \mathrm{t}$ have values close to those of human articular cartilage.

The increase in elastic modulus in the water filled samples, in comparison to the empty scaffolds, is explained by the water expulsion through the scaffold. The decrease in elastic modulus in the hydrogel filled samples is also explained by the water flow through the sample. The presence of a soft gel inside the pores makes the water flow easier and thus, increases the compliance of the scaffold. It is worth to notice that the situation is quite different from that of a scaffold with both macro and micro-pores. In previous studies with a Polycaprolactone scaffold filled with PVA hydrogel (Chapter 3) there was no difference in the elastic modulus for dry and water immersed scaffolds. The PCL scaffold is fabricated with a freeze extraction and particle leaching method (117) (123) (151) (121) (122) that generates both macro and micro-pores in the scaffold walls. The fact that the water immersed and dry PCL scaffold showed similar mechanical properties, whereas the macro-porous P(EA-HEA) scaffold showed a significant difference between the dry and water immersed samples reveals the role of micro porosity on water flow and mechanical properties. In the case of the macro and micro-porous PCL scaffold, a relative high 
Prediction of the in vivo mechanical behavior of biointegrable acrylic macroporous scaffolds

compression velocity makes the water expulsion from the pores fast and the modulus is similar for samples with and without water. The micro porosity seems to increase the water expulsion and hence, lower the elastic modulus. Considering that the P(EA-HEA) only have macro pores, water expulsion is not that fast and the elastic modulus increases. Once filled with a densely crosslinked hydrogel, the scenario is different, since all the pores are filled with hydrogel. The elastic modulus for the hydrophobic PCL increased significantly filled with PVA $6 \mathrm{f} / \mathrm{t}$ cycles, on the contrary the P(EA-HEA) scaffolds filled with PVA $6 \mathrm{f} / \mathrm{t}$ have the same modulus as the water immersed samples. This can be due to that hydrophilic P(EA-HEA) samples filled with hydrogel make the water expulsion easier, compared to hydrophobic PCL scaffolds. On the other hand when the micro pores are filled with densely crosslinked hydrogel, water expulsion is impeded and this double pore architecture can prevent water flow even more and increase the modulus.

These results are interesting since they indicate the performance of the scaffold once implanted in a chondral defect. Immediately after implantation, the scaffold pores would be filled with fluid and cells, a scenario similar to the water immersed scaffolds. When the tissue grow, the 
scaffold/tissue can be compared to the scaffold/hydrogel model for different cycles of $\mathrm{f} / \mathrm{t}$. Initially, the modulus of the P(EA-HEA) scaffolds would be similar to the water immersed scaffolds. Then, it would apparently decrease in the fist stages of tissue regeneration and with time increase, when the repair tissue inside the scaffold impede water flow through the porous structure.

\section{Conclusions}

This study evaluates the mechanical properties of P(EAHEA) scaffolds made with a porogen template technique for different crosslinking density. The scaffolds have been tested empty, water immersed or filled with PVA hydrogel subjected to different number of freezing and thawing cycles. It can be seen that the P(EA-HEA) scaffolds have increasing porosity with increasing crosslinking density, due to the solvent exchange in the synthesis method. Furthermore it can be observed that the PVA hydrogel enters all the scaffold pores, confirmed by porosity measurements and SEM photos. The PVA hydrogels show increasing crystallinity and decreasing water content with increasing number of freezing and thawing cycles. The SEM images show that the compression test do not damage or collapse the scaffold pores. The elastic modulus 
Prediction of the in vivo mechanical behavior of biointegrable acrylic macroporous scaffolds

increases with increasing crosslinking density for the P(EA-HEA) scaffolds. The results are interesting as they allow tailoring P(EA-HEA) scaffolds with different amount of crosslinking agent. When excess water is removed and the scaffolds are tested in dry conditions the modulus decreases, which show the effect of water inside the scaffold pores. When the scaffolds are filled with a soft hydrogel, the elastic modulus is in the same range as for the dried samples. The soft hydrogel facilitates water flow out of the scaffold during compression and the modulus decreases. When the hydrogel is more crosslinked, the modulus increases but only to values of water immersed scaffolds. This indicates that the water flow inside the scaffold pores is a key factor for determining mechanical properties. The results from hydrogel filled samples give a realistic approximation of the in vivo behavior of the scaffolds. PVA is a good cartilage model simulating ECM growth inside the scaffolds pores. The mechanical modulus for the P(EA-HEA)/PVA constructs is similar as for native articular cartilage. 
Chapter 5: Relationship between microporosity, water permeability and mechanical behavior of scaffolds for cartilage engineering

This chapter is based on the article "Relationship between micro porosity, water permeability and mechanical behavior in scaffolds for cartilage engineering" published in the Journal of the Biomechanical Behavior of Biomedical Materials 48, pages 60-60, 2015.

Polycaprolactone (PCL) scaffolds with a double micro and macro-pore architecture have been synthesized with the freeze extraction and particle leaching method. PCL concentrations of 15, 20 and 25\% PCL were used and tested in dry, water immersed and filled with PVA for 6 cycles of $f / t$. This study explores the influence of the micro-porosity of the pore walls on water permeability and scaffold compliance. Unconfined and confined compression tests were performed to characterize both the water permeability and the mechanical response of scaffolds with varying size of micro-porosity while volume fraction of the macro pores remain constant. SEM images reveal the morphology of the scaffolds. 
Relationship between micro-porosity, water permeability and mechanical behavior of scaffolds for cartilage engineering

\section{Results and discussion}

In the SEM images of the 15, 20 and 25\% PCL scaffold (Figure $5.1 \mathrm{a}-\mathrm{c}$ ) the macro porosity can be distinguished with a morphology similar to previous studies. (121) (122) (155) (123) The sizes of the macro and micro-pores have been estimated by measuring 6 macro and micro-pores from 3 different SEM pictures for each PCL scaffold. Macro pores are created when the porogen microspheres are extracted, and they are in range of $180 \pm 60 \mu \mathrm{m}$. The micropores are created in the freeze extraction process. The PCL solution and porogen spheres were mixed and rapidly frozen in liquid nitrogen. During freezing of the dioxane solution a solid-solid phase separation takes place and both the PCL and the dioxane crystallizes. In the case of PCL, a semicrystalline polymer is formed. The remaining polymer chains staying in the amorphous phase could contain some uncrystallized dioxane but most of the dioxane crystallizes in a separated phase. (240) (241) The frozen membrane was put into $\mathrm{EtOH}$ at $-20{ }^{\circ} \mathrm{C}$ and the dioxane crystallites leaches out. The micro-porosity comes from the cavities the dioxane leaves in the scaffolds. An increase in PCL/dioxane ratio decreases the micropore size. In a previous work PLLA scaffolds were made using 
the freeze extraction process and micro-pores in the scaffolds were measured manually with SEM photos and with Microtomography ( $\mu \mathrm{CT}$ ). The values obtained by SEM in that work are similar to those from the image analysis done from $\mu \mathrm{CT}$ scans. The $\mu \mathrm{CT}$ results therefore confirms the validity of SEM images analysis. (121) In this study the micro-porosity can be appreciated from the SEM pictures. It was observed that the micro-pore size diminishes with increasing PCL content. In figure 5.1d-f the pore walls of the different scaffold are seen. For the 15\% PCL the micropores are well distinguished and in the range of 2-10 $\mu \mathrm{m}$ (Figure 5.1d). In the photos of the 20\% PCL (Figure 5.1e) the micro-pores are still detectable but the size is hard to determinate with this level of magnification. In the $25 \%$ PCL (Figure 5.1f) the micro-pores are not visible; rather the pore walls appear more like roughness or abrasion. 
Relationship between micro-porosity, water permeability and mechanical behavior of scaffolds for cartilage engineering
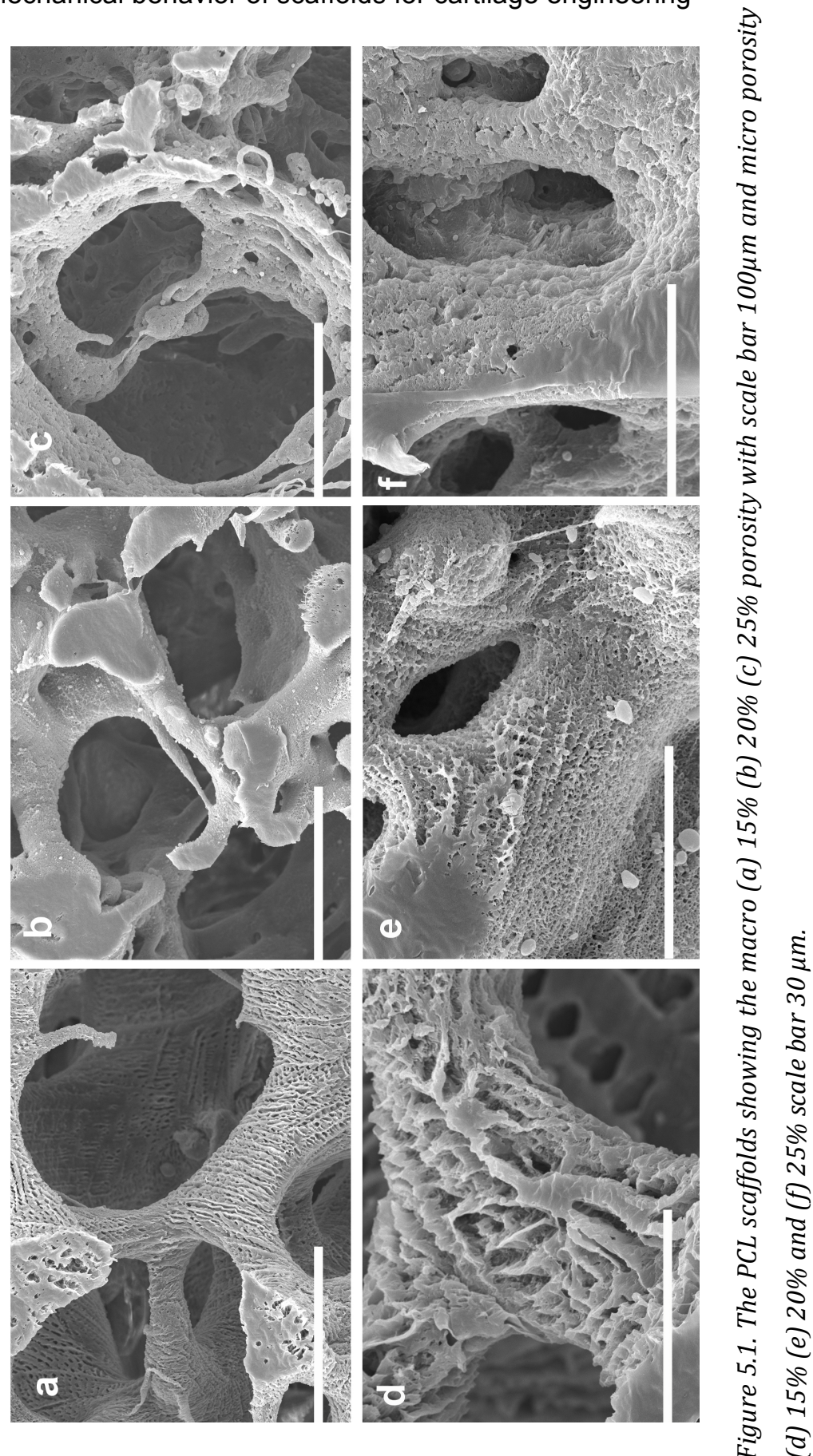

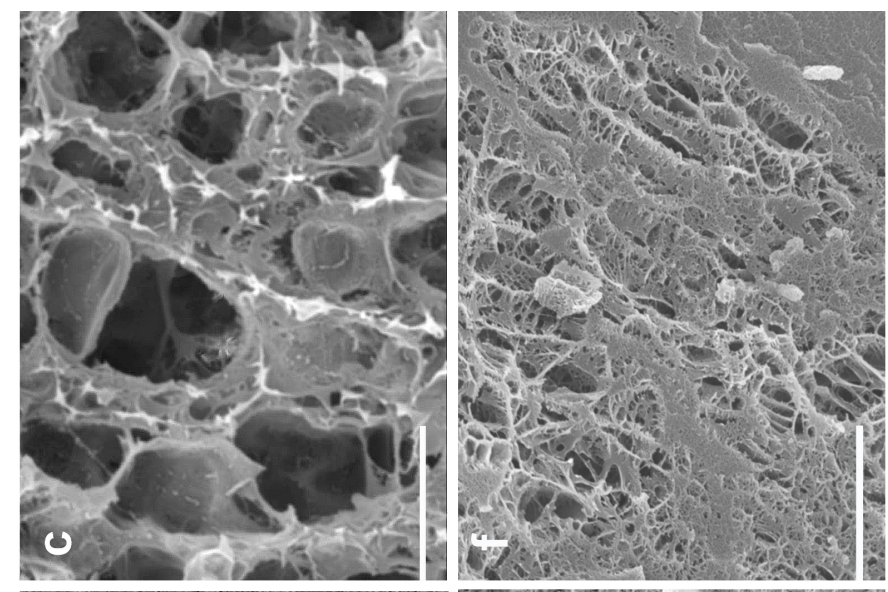

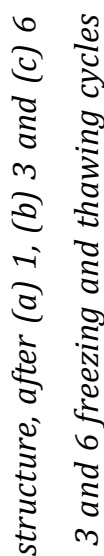
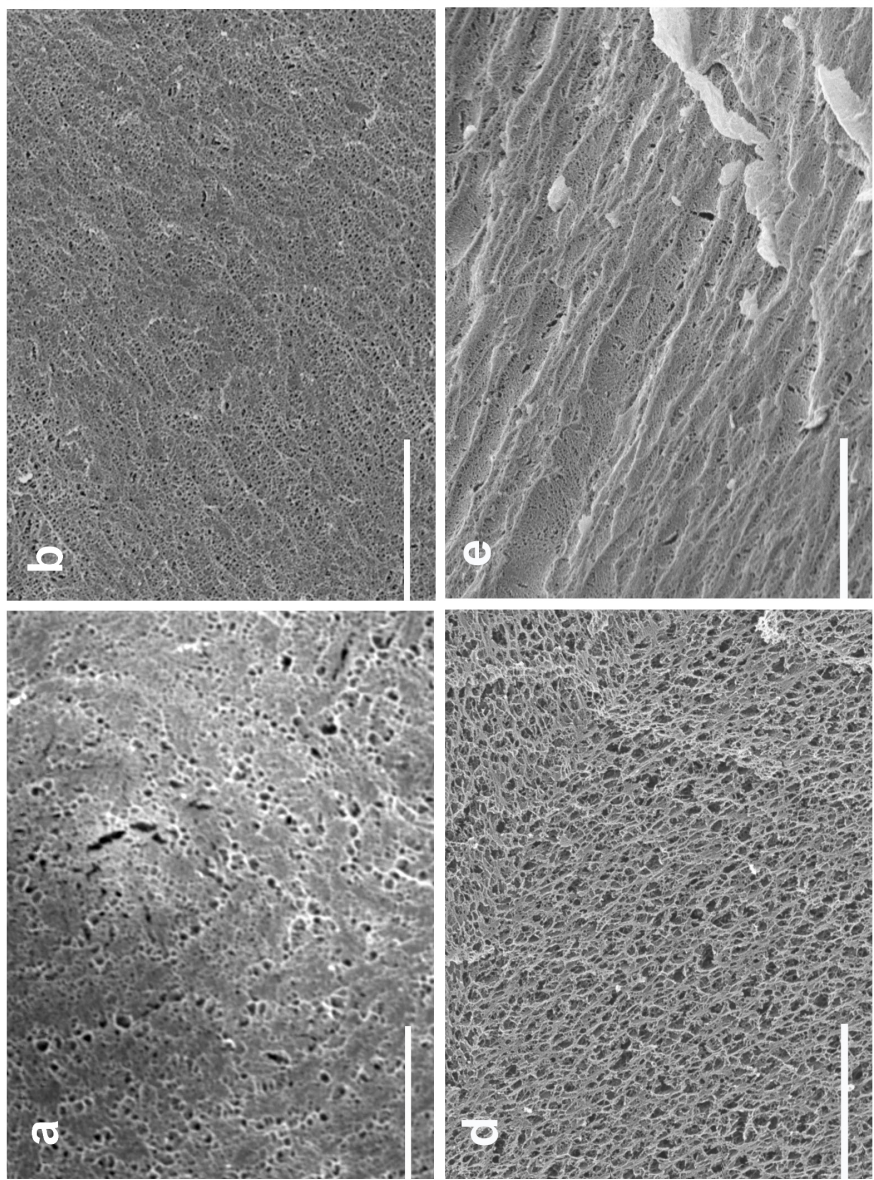

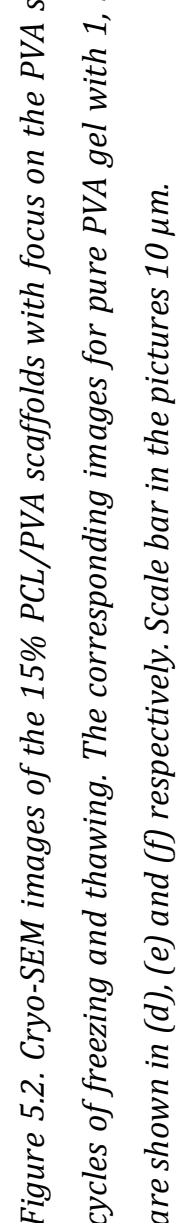


Relationship between micro-porosity, water permeability and mechanical behavior of scaffolds for cartilage engineering

Cryo SEM photos were taken for the 15\% PCL scaffold with the PVA hydrogel filling its pores for 1, 3 and 6 cycles of freezing and thawing (Figure 5.2 a-c). We have previously shown that the gel enters all macro and micropores of the PCL scaffold (Chapter 3) The calculated porosity according to equation 2 was zero, with a small standard deviation, which proves that filling the scaffold pores with PVA by vacuum is an effective method. The PVA gels are micro-porous and figure 5.2 shows the dependence of the freezing and thawing cycles of the porous structure on both the pristine PVA gels (Figure 5.2 d-f) and the PVA inside the PCL scaffold. There is not much difference between the pores of the PVA gel inside the scaffold after 1 and 3 cycles of freezing and thawing: the pore size is around $1 \mu \mathrm{m}$. For 6 cycles of freezing and thawing the pore size of the PVA gel increases and there is a dispersion of pore sizes ranging from 1 to $10 \mu \mathrm{m}$ (Figure 5.2 c) with a similar behavior shown by the pure PVA gels (Figure 5.2d-f). The pores are larger inside the scaffold than in the pure PVA gels. The limitation imposed by PCL trabeculae on contraction and expansion of PVA during freezing and thawing could have some influence on the formation of micro-pores. 
Confined compression tests were done to evaluate the permeability of the pure PVA gel and the 15\% PCL/PVA construct. The 20 and 25\% PCL scaffolds filled with PVA and subjected to 6 cycles of freezing and thawing were measured and compared to the scaffolds immersed in water. Figure 5.3 shows the stress-strain curve for one of the scaffolds: $20 \%$ PCL immersed in water, from with the aggregate modulus and permeability was calculated. The confined compression test consisted of a series of successive strain ramps to 2, 4, 8, 12, 16 and $20 \%$ of strain. Each new strain level was followed by a relaxation period of $15 \mathrm{~min}$. The values of the stress after the relaxation period was best fitted to a straight line and the aggregate modulus was obtained from its slope (Figure 5.3a). For the stress relaxation at $8,12,16$ and $20 \%$ of strain a forcetime graph was drawn (Figure 5.3b shows the force-time graphs for each of the stress relaxation strains). The relaxation time was calculated from best fit to a single exponential function for large times. The permeability was calculated from equation 4. From this it can be seen that permeability of the pure gel is much higher than that for the 15\% PCL/PVA construct (Figure 5.4a). For one cycle of freezing and thawing permeability of the pure PVA gel is very high, since the gel has limited crosslinks. For each cycle of freezing and thawing the crosslinking density is 
Relationship between micro-porosity, water permeability and mechanical behavior of scaffolds for cartilage engineering

increasing and the gel gets stiffer. (Chapter 3) The permeability is decreasing until 4 cycles of freezing and thawing where the values are in the same range for 5 and 6 cycles of freezing and thawing. This can be due to that the hydrogel after 4-6 cycles of freezing and thawing have similar crystallinity, or pore size due to the freezing process. (168) For the 15\% PCL/PVA construct permeability does not change with the number of cycles of freezing and thawing. This implies that the water flux inside a hydrogel filled scaffold is mainly controlled by the morphology of the scaffold, and less influenced by the crosslinking density of the hydrogel.

(a)

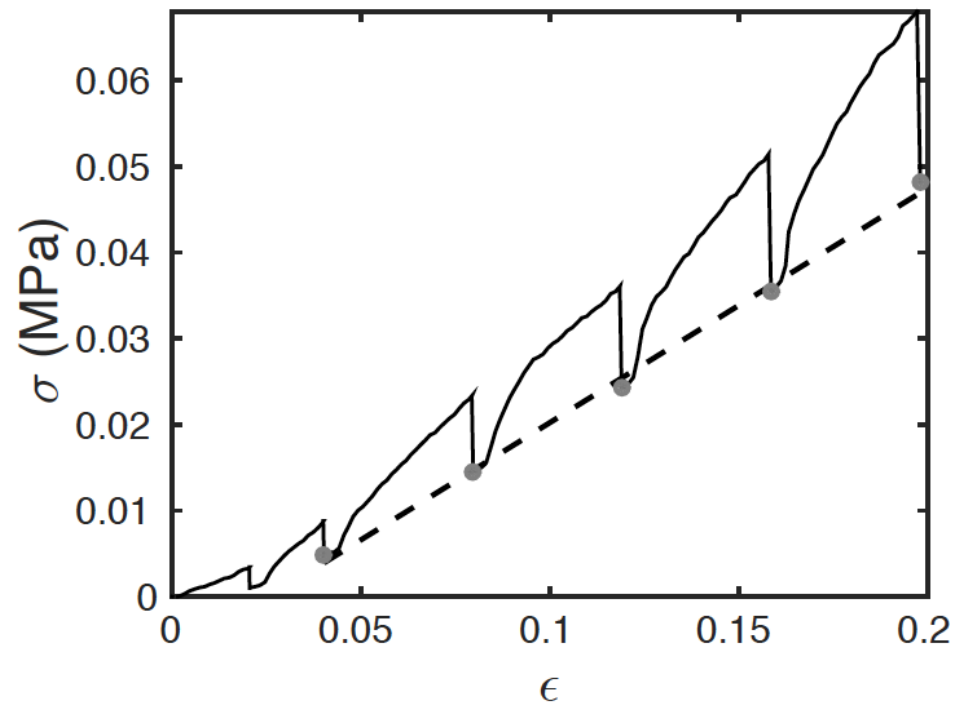


(b)
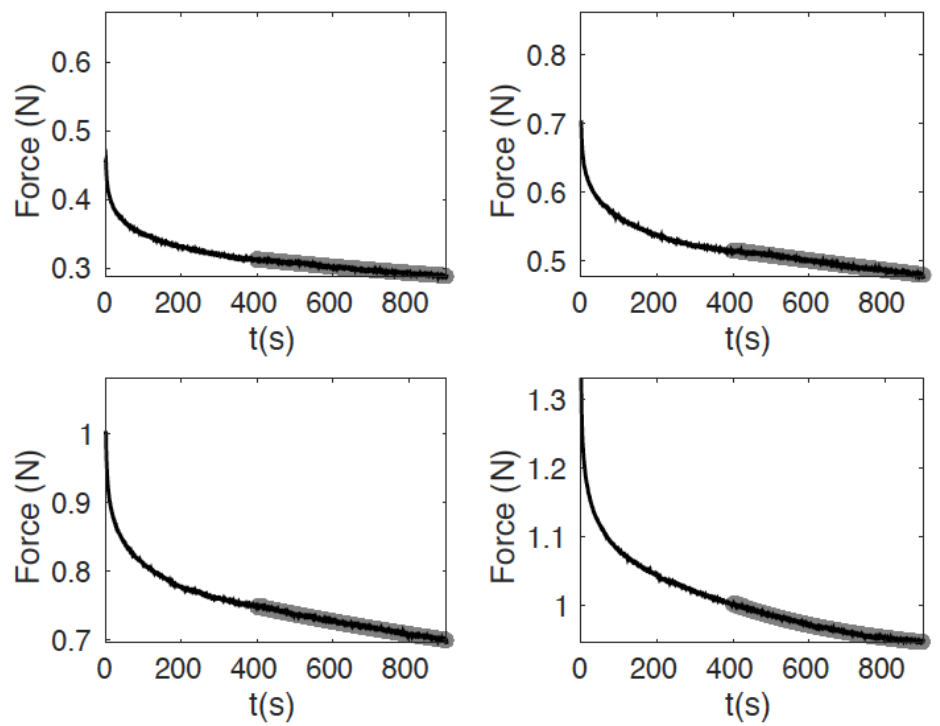

Figure 5.3. (a) A representative stress-strain graph from the confined compression assay for 20\% PCL immersed in water. The aggregate modulus is obtained as the slope from the linear fit of the stress-strain curves at equilibrium points (gray circles and dashed line). (b) The force-time graphs with strain of 8,12, 16 and 20\% represented from top left to right down. The relaxation time is calculated from the fitting to a single-exponential function for large time (thick grey line). The permeability is deduced from equation (4) with the values obtained of the aggregate modulus and relaxation time. 
Relationship between micro-porosity, water permeability and mechanical behavior of scaffolds for cartilage engineering

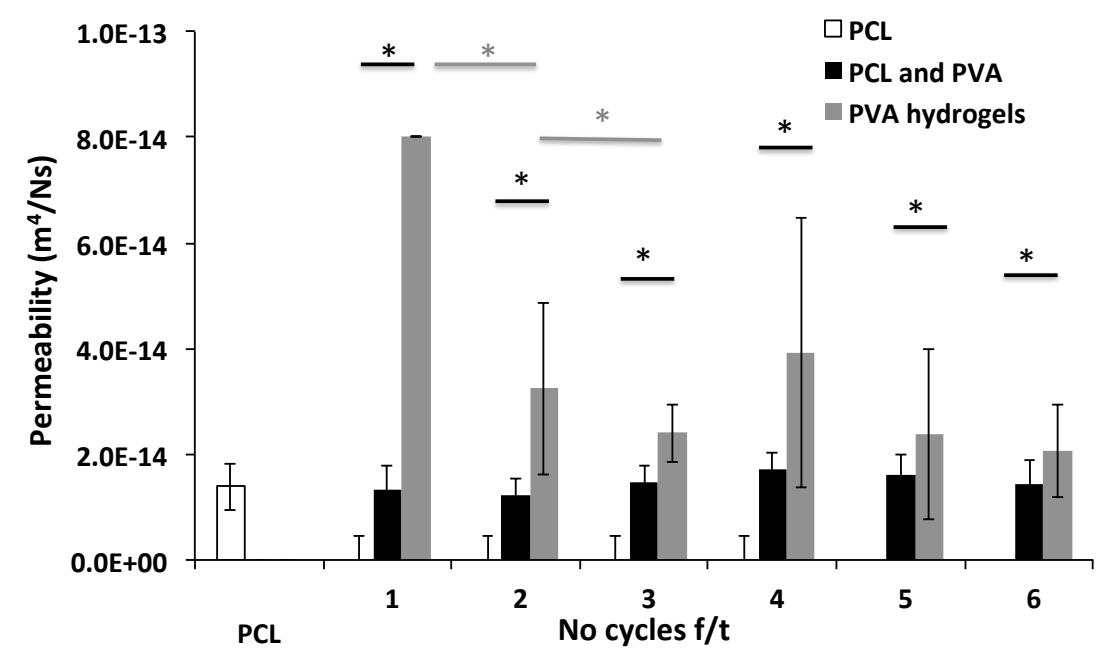

5.4a)

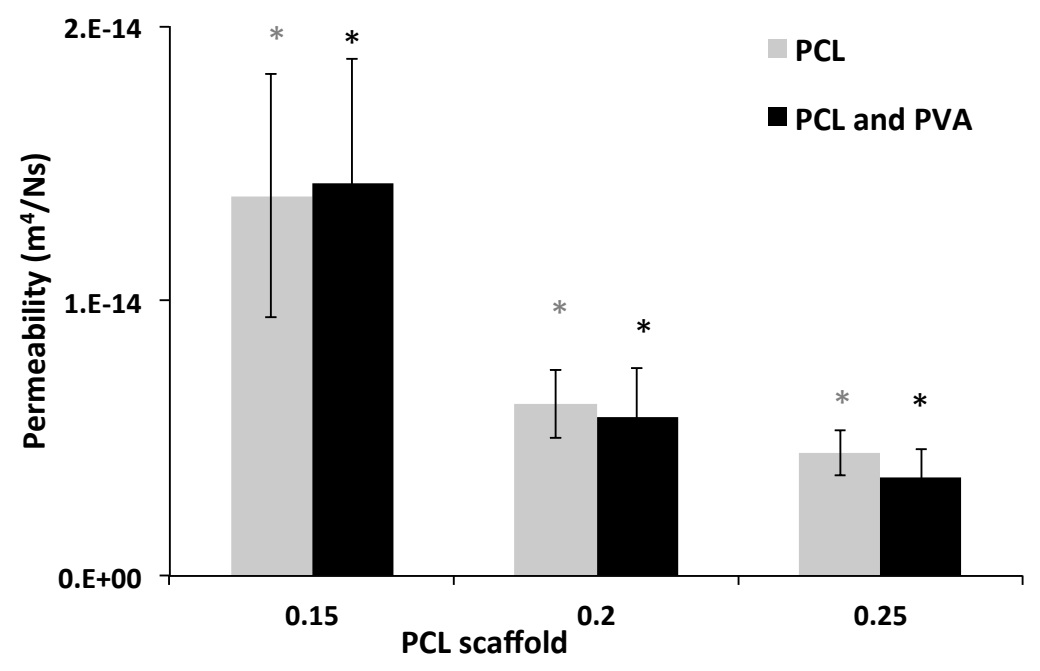

$5.4 b)$ 


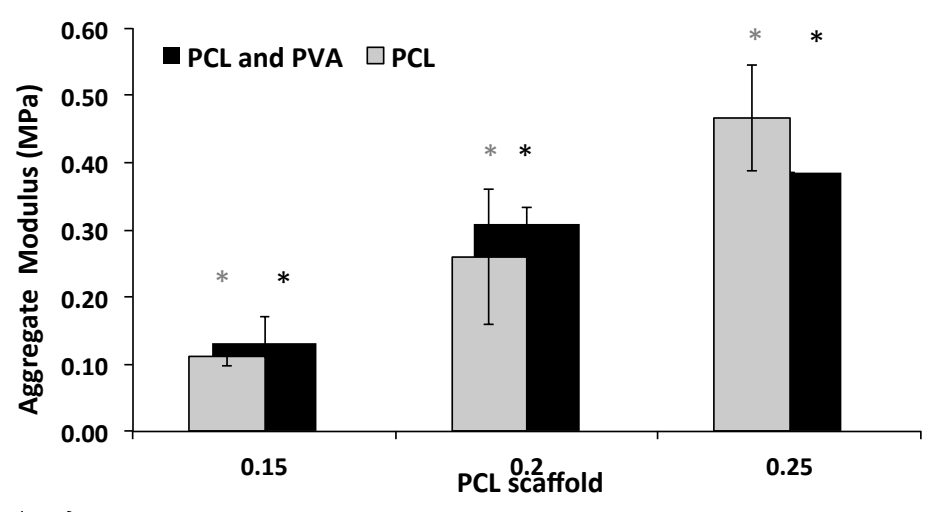

$5.4 c)$

Figure 5.4. (a) Permeability for pure PVA and 15\% PCL immersed in water and filled with PVA for 1 to 6 cycles of freezing and thawing $(f / t)$. An ANOVA statistical test was done, and when statistical difference was found an unpaired t-test was performed to evaluate the statistical difference between tested groups with $p<0.05$. Black asterisk show statistically significant difference between pure PVA gels and PCL/PVA constructs, and grey asterisk between groups of pure PVA gels different cycles $f / t$. b) Permeability and c) aggregate modulus for series of PCL scaffolds with and without PVA filling. The black asterisk show statistically significant difference between PCL/PVA scaffolds and grey asterisk between PCL scaffolds. There are no statistically significant differences between PCL scaffolds and PCL/PVA constructs.

As seen in figure 5.1 the scaffold is composed of both macro and micro-pores. To evaluate how the scaffold architecture influences the permeability, confined compression tests were performed on PCL/PVA scaffolds with different micro-porosity after 6 cycles of freezing and thawing. In figure $5.4 \mathrm{~b}$ it is seen that for increasing PCL 
Relationship between micro-porosity, water permeability and mechanical behavior of scaffolds for cartilage engineering

content, i.e. decreasing micro-porosity, the permeability decreases, showing the effect of the micro-porosity on the water flux through the scaffold trabeculae. There is no statistically significant difference between the permeability of the PCL scaffold and the PCL/PVA construct for 6 cycles of freezing and thawing for any of the PCL scaffold series (Figure 5.4b). This indicates that the water permeability is foremost decided by the microporosity in the pore walls. These results make it reasonable to think that the water flux through a scaffold filled with cells and ECM (here the filling is simulated by the PVA hydrogel) is mainly controlled by the microporosity of the scaffold, and less influenced by the macro porosity or tissue density. It is worth to notice that the aggregate modulus for the 15\% PCL/PVA construct is constant throughout 1-6 cycles of freezing and thawing (Figure 5.6). This could imply that the aggregate modulus is the value to compare to the permeability, and not the elastic modulus (Chapter 3). Neither permeability nor aggregate modulus change for the different number of $f / t$ cycles of the PCL/PVA construct (Figure 5.4a and 5.6). The elastic modulus however, is affected by the hydrogel density (Chapter 3). 
Figure 5.4c shows the results for the aggregate modulus of PCL scaffolds with different micro-porosity with and without hydrogel filling. The modulus increases for decreasing micro-porosity and there is no significant difference between the scaffold with or without PVA. The aggregate modulus for human articular cartilage is around 0.5-0.9 MPa (228) and the permeability values typically range between $10^{-15}$ and $10^{-16} \mathrm{~m}^{4} / \mathrm{Ns}$. (41) (42) (243) (244) (25) In these terms the PCL/PVA construct can be considered a good cartilage model, specially the 25\% PCL/PVA. The hydraulic pressure in cartilage depends on the proteoglycans in the ECM and is determined by pore size, pore distribution, tissue composition and mechanical deformations.

Loading and unloading tests in unconfined compression mode were performed to determine the apparent modulus and maximum stress reached for $15 \%$ of deformation for the different samples (Figure 5.5). A compression test until total collapse of the samples was also done to see the different zones of the stress-strain curve (Figure 5.7). The typical stress-strain zones are the elastic region corresponding to low strain, the plateau region where the scaffold struts undergo permanent deformation and the high slope stress-strain region which shows the behavior 
Relationship between micro-porosity, water permeability and mechanical behavior of scaffolds for cartilage engineering

of the compact material. (65) The values for the apparent modulus and the maximum stress are increasing for increasing PCL content, i.e decreasing micro-porosity, for the water filled and PVA filled samples. The elastic modulus of rabbit articular cartilage is $0.41 \pm 0.12 \mathrm{MPa}$ (152) and of human articular cartilage 0.581 $\pm 0.168 \mathrm{MPa}$. (25) From the results it can be seen that all the water immersed scaffolds have values close to the animal model and the scaffolds filled with PVA gel have values similar to human articular cartilage. In the loading of the PCL samples immersed in water (Figure 5.7), the samples with higher PCL content have less distinguished pore collapse zones. (65) This is even more pronounced when the pores are filled with PVA gel. In none of the samples trabeculae buckling and pore collapse is seen and for the curves of the samples with higher PCL content higher stress is required to compress the scaffold until the same strain. 

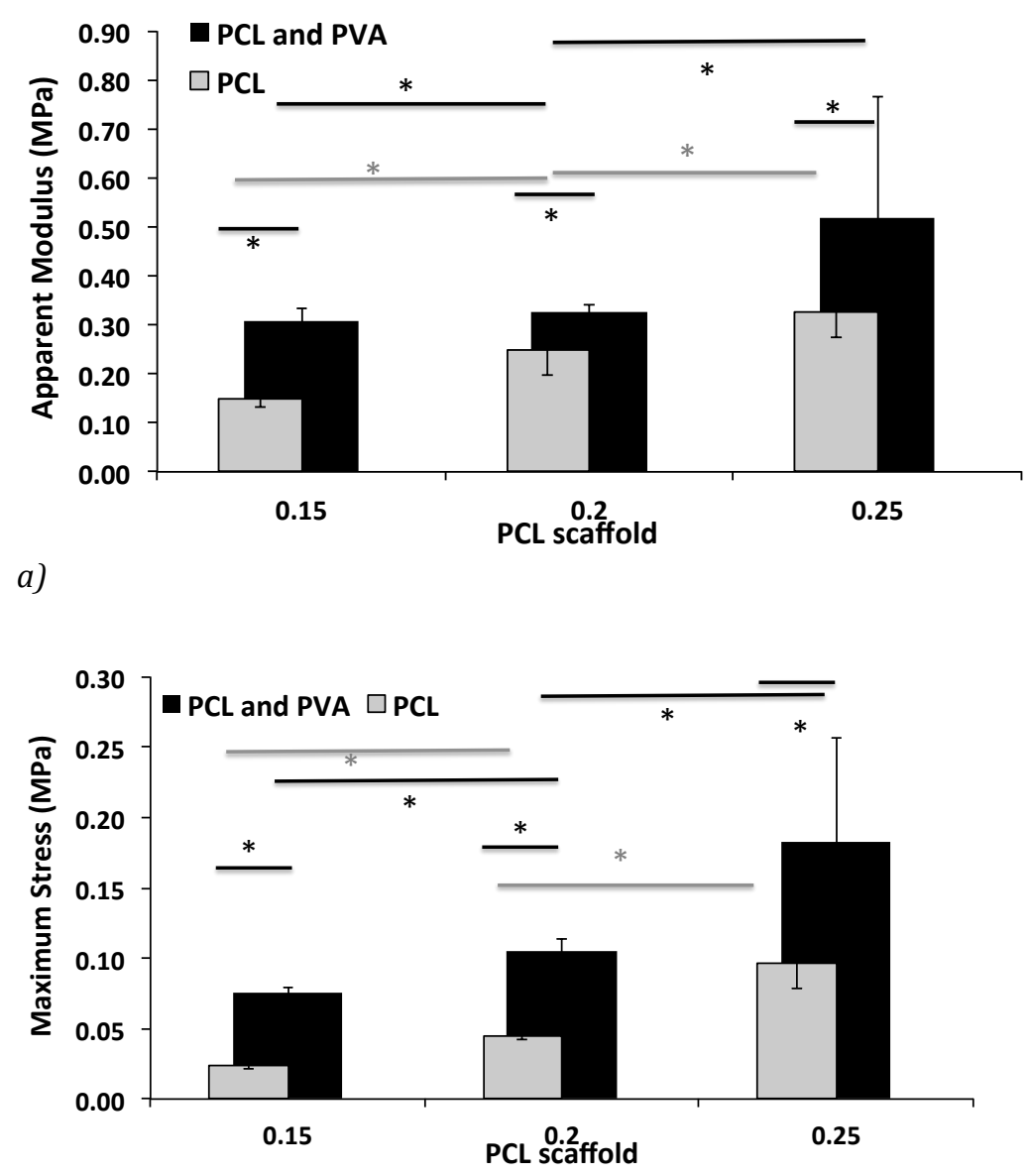

b)

Figure 5.5 a) Apparent modulus for the series of PCL and PCL and PVA scaffolds. b) Stress at 15\% strain for the series of PCL/PVA scaffolds. An ANOVA statistical test was done, and when statistical difference was found an unpaired t-test was performed to evaluate the statistical difference between tested groups with $p<0.05$. A black asterisk indicates statistically significant difference between the empty and filled scaffolds and between the PCL/PVA scaffolds. Grey asterisk indicate statistically significant difference between the empty PCL scaffolds. 
Relationship between micro-porosity, water permeability and mechanical behavior of scaffolds for cartilage engineering

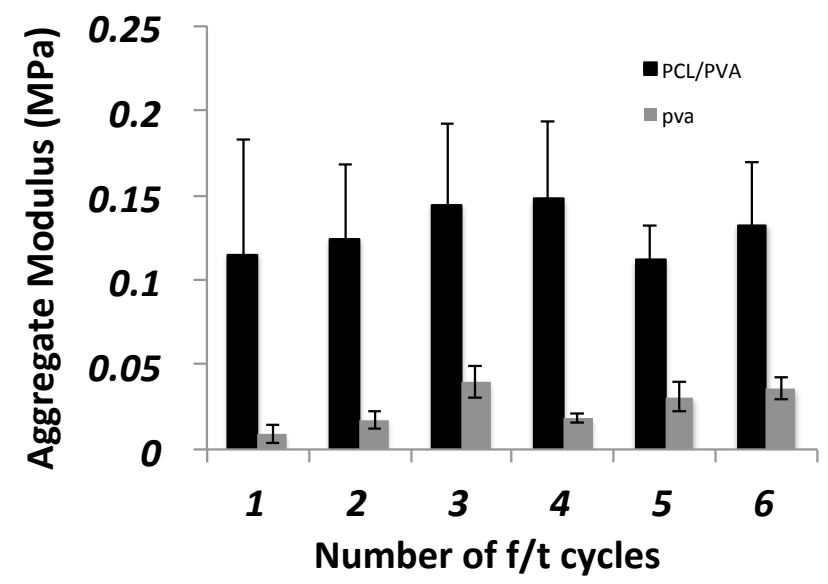

Figure 5.6 The aggregate modulus for the 15\% PCL/PVA (black) and PVA hydrogel (grey) for 1-6 cycles f/t.

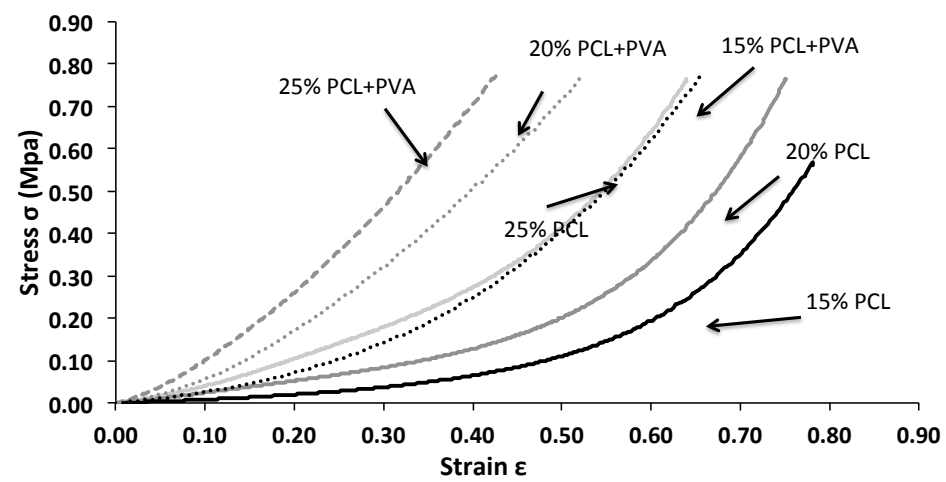

Figure 5.7. The loading of the PCL scaffold without (continuous line) and with PVA gel 6 cycles of $f / t$ (dashed line). The black lines is 15\% PCL, the dark grey lines 20\% PCL and the light grey lines 25\% PCL. 
Unconfined stress relaxation tests to $5 \%, 10 \%$ or $15 \%$ compressive strain were performed on all the different PCL, PCL/PVA scaffolds and the pure PVA hydrogel. Figure 5.8 shows the stress-relaxation curves obtained for the $15 \%$ PCL scaffold, with a strain of $15 \%$. The stress relaxation curves for 5 and $10 \%$ strain show the same behavior and are not shown. The experiments were performed with the samples immersed in water at room temperature. At this temperature stress relaxation of a PCL scaffold is quite a slow process. Indeed, in the performed experiment the stress was always far from equilibrium; reaching equilibrium would require an experiment several decades longer. In previous study (Chapter 3) it was shown that the apparent modulus of PCL scaffold immersed in water did not reach values higher than that of the dry sample. This means that when the scaffold is deformed in free compression mode, water leaves the pores faster compared to the deformation rate and the mechanical behavior is dominated by the viscoelasticity of the porous polymer (i.e. by conformational rearrangements of the polymer chains and sliding of polymer crystallites). In the case of PVA hydrogel the values of the stress for the same strain are lower than that of the PCL but in the same order of magnitude. The stress relaxation process is still quite slow. 
Relationship between micro-porosity, water permeability and mechanical behavior of scaffolds for cartilage engineering

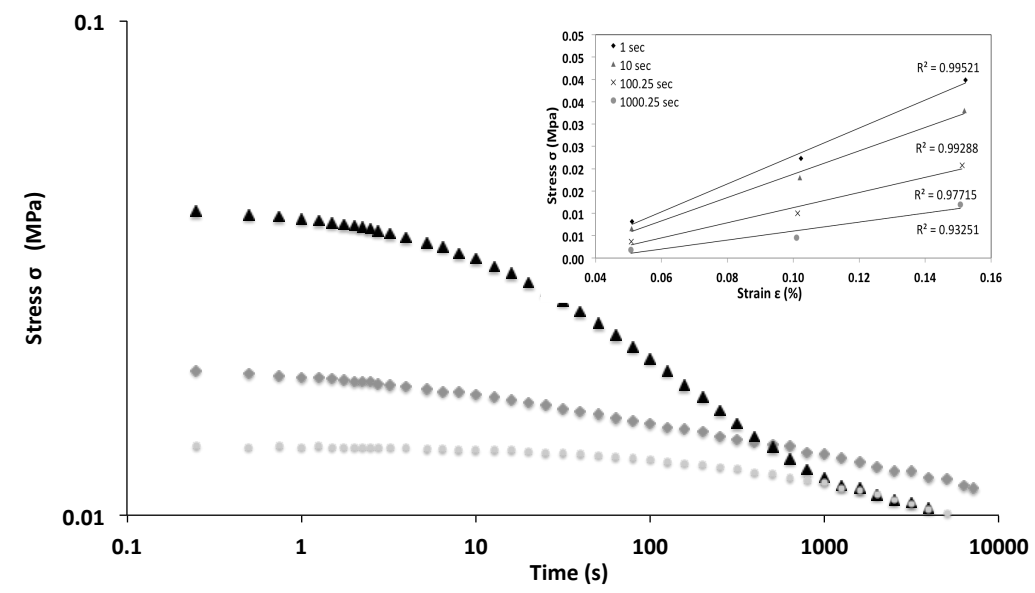

Figure 5.8. The logarithmic stress relaxation graphs from the compression test for the 15\% PCL scaffold without (grey diamonds) and with (black triangles) PVA and the pure PVA hydrogel (light grey circles) for 15\% of deformation. The inset shows a linearity test of the results.

Interestingly enough, the stress measured at short intervals in the composite is much higher than for the PCL scaffold or the PVA hydrogel, showing the synergic effect produced by a hindering effect of the composite structure on water permeation under compression loading. Since water is incompressible, any delay of water-flow out of the sample during compression produces an important increase in the recorded stress. In fact, the water-flow though the PVA gel should be hindered by the tortuosity imposed by the trabecular structure of the hydrophobic PCL, giving rise to a delay in water-flow out of the scaffold/gel. Due to hydrogel filling of the PCL scaffold, 
water-flow in the composite is expected to be slower than in the empty scaffold. Thus, the effect is an initial stiffness of the composite higher than seen in any PCL scaffold or PVA gel. The stress relaxation follows with a progressive decrease in the stress with time. The relaxation process for the PCL/PVA construct is faster than for the single components scaffold or hydrogel, which shows that the kinetics of the relaxation process it not controlled by the polymers itself, but by water inside it. The results must be interpreted bearing in mind that the observed phenomenon is predominated by the water-flow inside the sample and not by the polymer rearrangements. This means that a deformation of the sample is progressively allowed with water-flow out of the sample.

\section{Conclusions}

This work uses an experimental model to demonstrate the effect of micro-porosity on the mechanical properties of a macro and micro-porous PCL scaffold for cartilage engineering. The growing cartilaginous tissue is simulated using a PVA hydrogel with tailored elastic modulus. Confined compression tests show a constant permeability in the PCL/PVA scaffolds with varying hydrogel density, revealing that the permeability is non-dependent on 
Relationship between micro-porosity, water permeability and mechanical behavior of scaffolds for cartilage engineering

hydrogel filling. To assess this determining variable, the micro-porosity in scaffold was changed and results revealed that permeability decreases with reduced microporosity. Furthermore, no permeability changes were observed in hydrogel-filled and water-filled scaffolds. This signifies that permeability and ultimately water flow is principally dependent on the scaffold's micro-porosity. The unconfined compression test shows that the stress relaxation behavior of the scaffold and hydrogel construct is a synergic effect produced by the scaffold, hydrogel and water flow. The results of this experiment highlights the importance of water mobility in scaffolds. The mechanical behavior of the scaffold implanted in a cartilage defect will depend on the water flow inside the growing tissue and the scaffold pores. The permeability for natural articular cartilage is between $10^{-15}$ and $10^{-16}$ (41) (42) (243) (244) (25) and the values reached for the PCL/PVA model are between $1.4 \cdot 10^{-14}$ and $3.5 \cdot 10^{-15}$ for increasing PCL content. The elastic modulus for natural articular cartilage is around 0.5-0.9 MPa (228) and the values reached for the PCL/PVA model were between 0.3-0.5 MPa. This study, with the limitations of using a hydrogel filling the scaffolds pores instead of extracellular matrix molecules produced by cultured chondrocytes, can be extrapolated to the 
behavior of the scaffold implanted in an articular cartilage defect. 
An experimental fatigue study of a porous scaffold for the regeneration of articular cartilage

\section{Chapter 6: An experimental fatigue study of a porous scaffold for the regeneration of articular cartilage}

This chapter is based on the published article "An experimental fatigue study of a porous scaffold for the regeneration of articular cartilage" in The Journal of Biomechanics Volume 48 Issue 7 pages 1310-1317, 2015.

This experimental study aim to predict the long-term mechanical behavior of a porous scaffold implanted in a cartilage defect for tissue engineering purpose. Fatigue studies were performed by up to 100,000 unconfined compression cycles in a Polycaprolactone scaffold with highly interconnected pores architecture. The PCL scaffold was fabricated with freeze extraction and particle leaching technique for 15\% PCL concentration and 1:1 PEMA/PMMA. The scaffold compliance, stress-strain response and hysteresis energy have been measured after different number of fatigue cycles, while the morphology has been observed by scanning electron microscopy at the same fatigue times. The scaffolds were tested in dry and water immersed conditions and filled with PVA for 6 cycles of $f / t$. DSC scan was done to evaluate the effect of water to the PCL scaffolds. 


\section{Results and discussion}

The PVA hydrogels were examined to evaluate the water content of the gels. Hydrogels were fabricated from the aqueous PVA solution and exposed to 6 cycles of freezing and thawing. The hydrogels were freeze dried, and then heated in a TGA scan. After the freeze drying, the average water content of the hydrogels, was obtained as the difference in mass of the hydrogel and the dry samples obtained. The average water content was calculated to $86.73 \pm 1.85 \%$. Then, the samples were examined in TGA, and the resulting heating curves show a mass loss of $4.12 \pm$ $0.29 \%$ up to $180^{\circ} \mathrm{C}$ that was assigned to the remaining water. In this way the PVA hydrogels contain $87.2 \%$ water based on initial weight of the samples, showing very little water loss during thawing.

Before mechanical testing the dry PCL scaffold has micro, $(10 \pm 5) \mu \mathrm{m}$, and macro, $(180 \pm 60) \mu \mathrm{m}$ pores (Figure 1a). These mean pores size were obtained by measuring the size of 10 micro and macro pores in 3 different SEM photos. The structure is similar to the ones obtained in previous works. (121) (Lebourg, Suay Antón, and Gómez Ribelles 2010) (122) (Lebourg et al. 2013) Without fatigue, the structure of the dry and immersed scaffold is 
An experimental fatigue study of a porous scaffold for the regeneration of articular cartilage

very similar. Not until 10,000 cycles of fatigue a change is seen (Figure 6.1f-1g), between the dry and immersed samples. The dry scaffold is clearly affected by the dynamic loading applied, the pores are collapsed and the overall scaffold structure is changed in comparison to earlier fatigue times (Figure 6.1f). For the immersed scaffold the fatigue effect is not that comprehensive (Figure 6.1g), the scaffold trabeculae are not broken but a compressive effect exerted by the load is noticed, as the pores become more oval shaped. The dry scaffold after 100,000 cycles of fatigue (Figure 6.1h) is now almost totally collapsed and many trabeculae are broken (see the arrow in Figure 1h). In the immersed samples after 100,000 cycles (Figure 6.1i) the pores are compressed but have not collapsed to the same extent as in the dry samples. The difference between the dry and immersed scaffolds shows the effect of the water inside the pores of the scaffold. The water inside the pores acts as unitary tensors with forces working against the compressive force and the applied load is not as damaging as for the samples without liquid. 


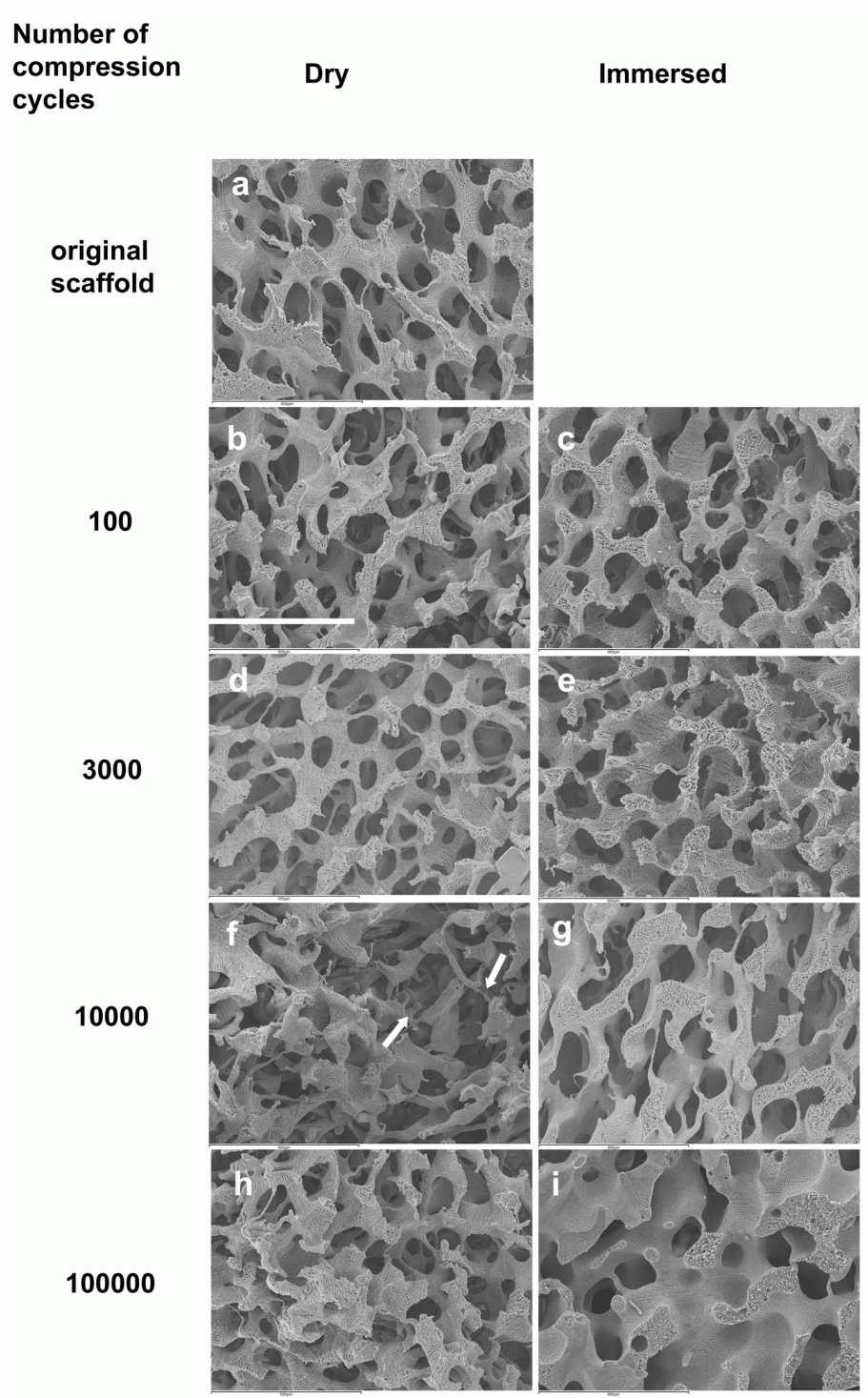

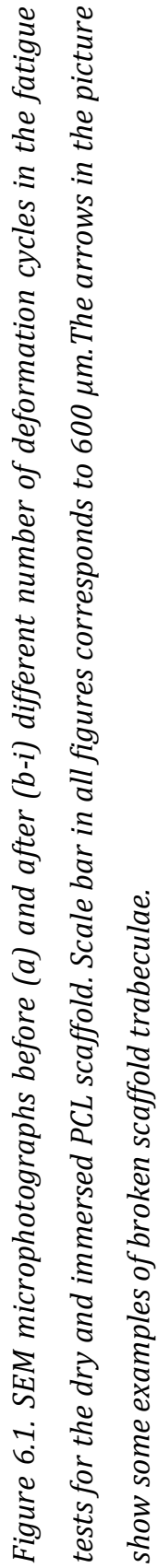


An experimental fatigue study of a porous scaffold for the regeneration of articular cartilage

This effect is even more emphasized for the PCL scaffold filled with PVA gel. It is worth to notice that the PVA solution enters both the micro and macro pores of the scaffold, forming a continuous phase that fills all scaffold voids. The calculated porosity according to equation 3 was zero, which probes the effectiveness of the vacuum filling of the PVA solution. No pore size change of the PCL scaffold is expected during the freezing and thawing step, the water inside the pores freezes and expands but during the thawing a retraction is expected. The porous honeycomb structure of the PVA gel is seen inside the pores of the PCL before fatigue in Figure 6.2a and 6.2c. The morphology of the PCL scaffold filled with PVA gel remains unchanged during the fatigue test (Figure 6.2b). 

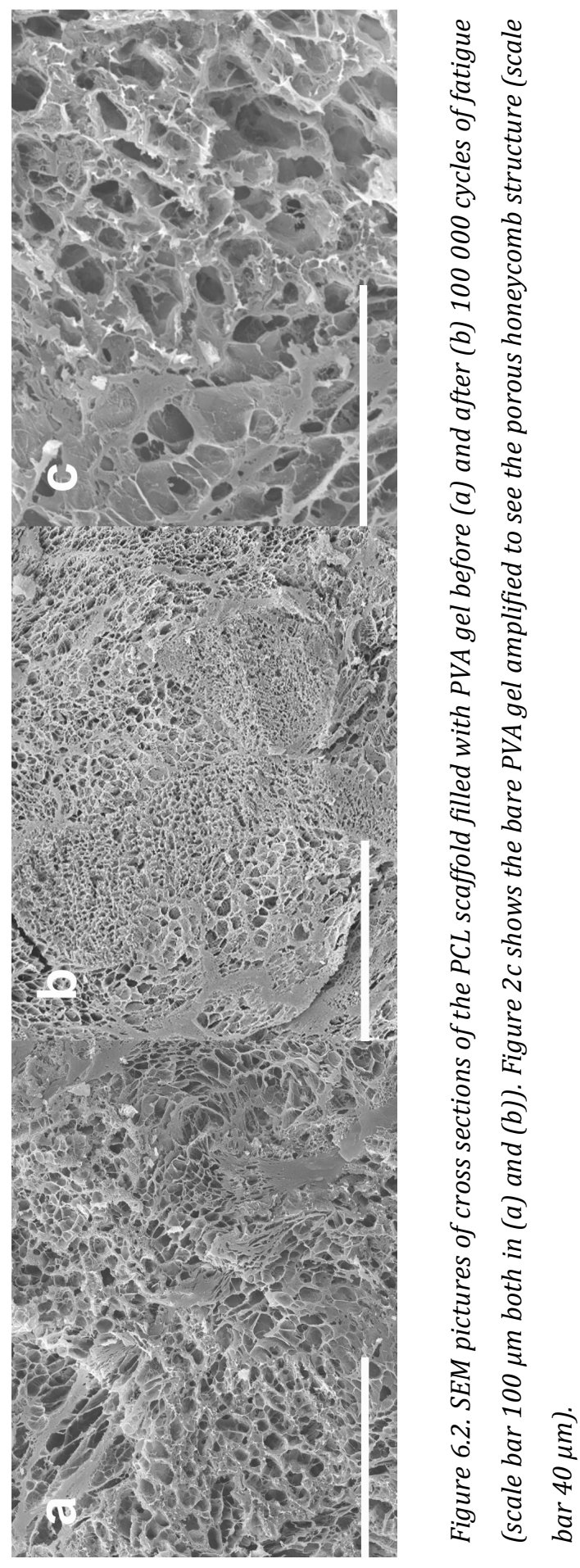
An experimental fatigue study of a porous scaffold for the regeneration of articular cartilage

The DSC heating scan is represented in figure 6.3 and shows the graph of the heat flow and temperature for the dry and water immersed PCL samples. The calculated crystallinity is $45 \%$ for both the samples and the melting peak is 3 degrees lower for the water immersed sample. The $\mathrm{T}_{\mathrm{g}}$ is around $-60{ }^{\circ} \mathrm{C}$ for both the dry and immersed sample with a broad transition and low heat capacity increment due to the high crystallinity. A large fraction of the polymer chains that do not participate in the crystallites is immobilized in an amorphous rigid phase and hence do not participate in the glass transition. This feature seems to be enhanced by freeze extraction. (245) (246)(Lebourg, Suay Antón, and Gómez Ribelles 2008) The plasticizing effect of the water adsorbed in the amorphous phase (247) of the polymer could not be quantified. This effect is considered very small comparing the effect of the mobility of the water inside the macro pores of the scaffold, supporting the compression loads. 


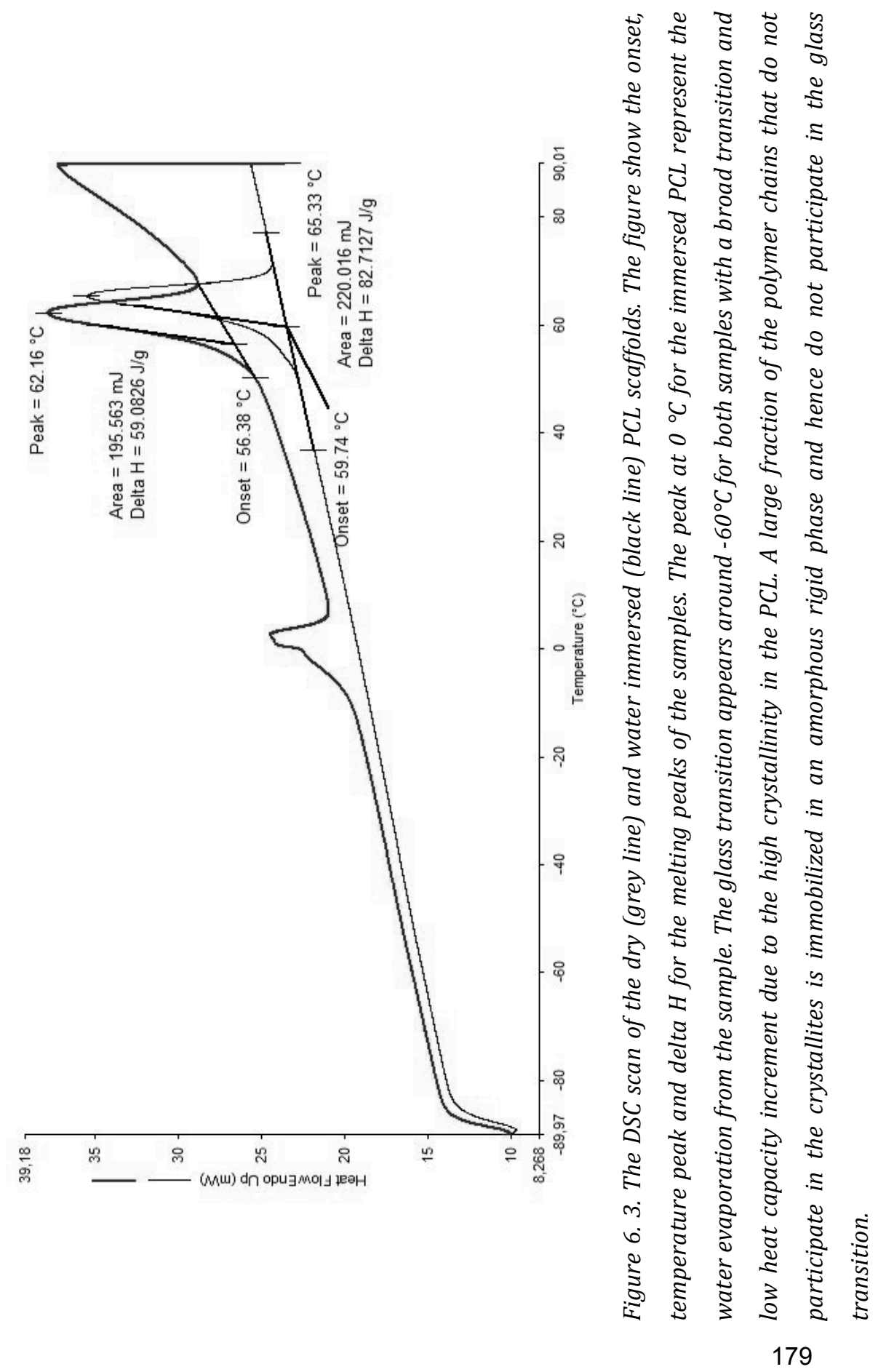


An experimental fatigue study of a porous scaffold for the regeneration of articular cartilage

The PVA gels are showing an almost perfect elastic stress strain response with loading and unloading paths that nearly overlap (Figure 6.4a). Furthermore no difference is seen before and after 100,000 cycles of fatigue. The dry and immersed PCL scaffolds (Figure 6.4b and 6.4c) show a different result, with a clear difference in the first and second loading path before fatigue. After 100,000 cycles of fatigue the curves are moved to smaller strains and the loading and unloading paths are much closer to each other indicating a more compact material where the pores have collapsed. The PCL/PVA construct shows (Figure 6.4d) a result combining the elasticity of the PVA gel and the stiffness of the PCL scaffold. It is seen a certain permanent deformation in the PCL scaffolds. In the PVA gel the strain is returning to its original state of zero displacement after unloading the charge. For the dry PCL scaffold the unloading curve reaches values of about 15\% deformation and the water immersed scaffolds of about $12 \%$ deformation. This difference could be due to the water inside the pores of the scaffolds. Water is being pushed out of the scaffold pores but is also able to be reabsorbed in the cyclic compressive assays. For the PCL and PVA construct the permanent deformation is around 3\%. 

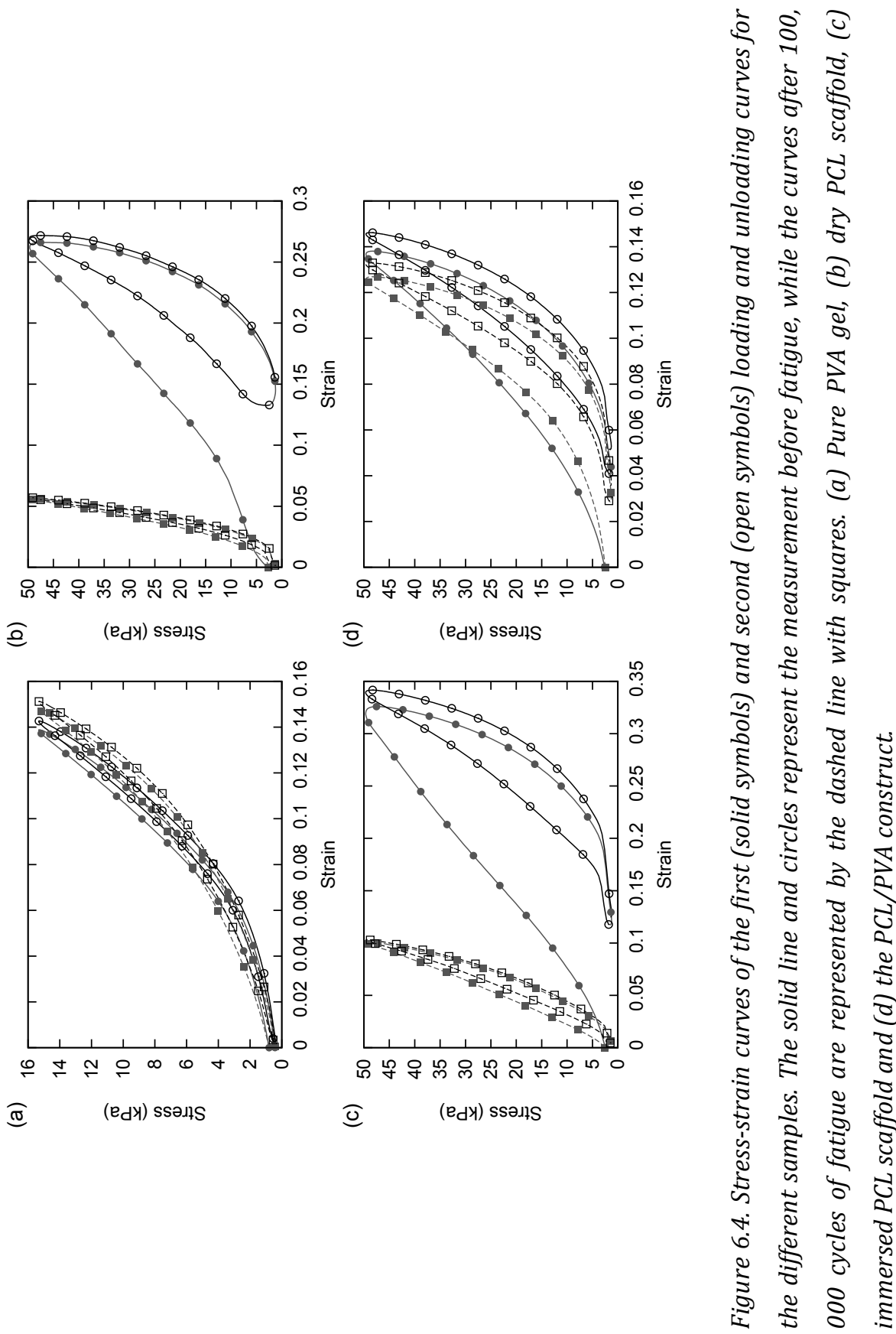

कृ (edy) ssajis

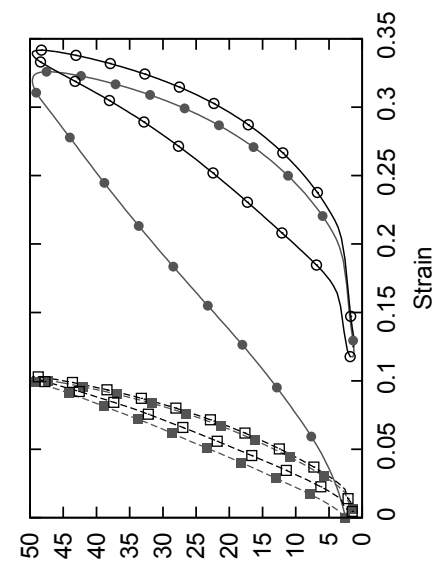

o

(edy) ssans 
An experimental fatigue study of a porous scaffold for the regeneration of articular cartilage

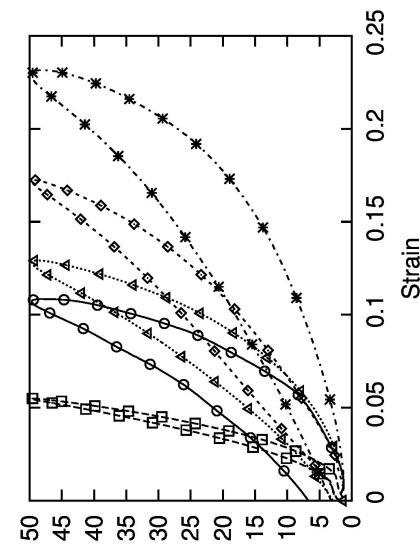

อ

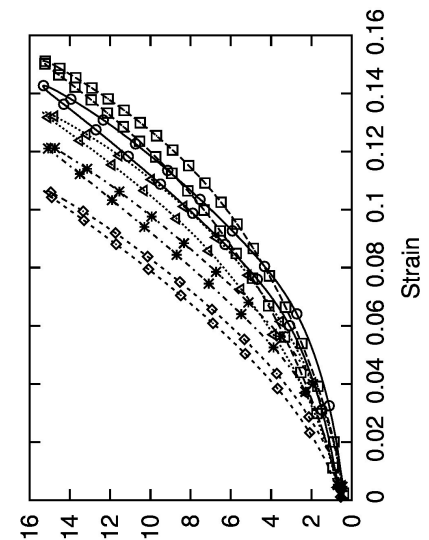

తิ

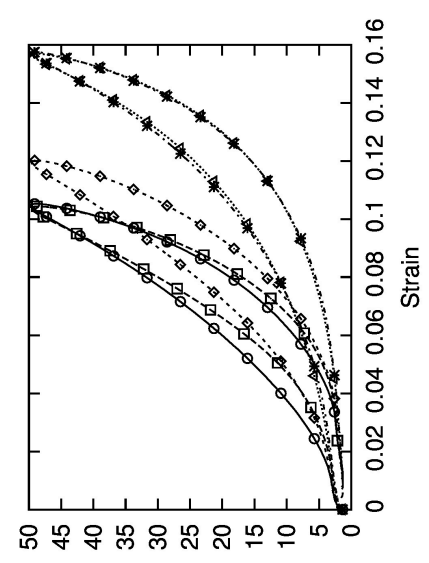

흐

$\left(\operatorname{edy}_{\mathrm{d}}\right) \operatorname{ss} 2 \pi \mathrm{s}$

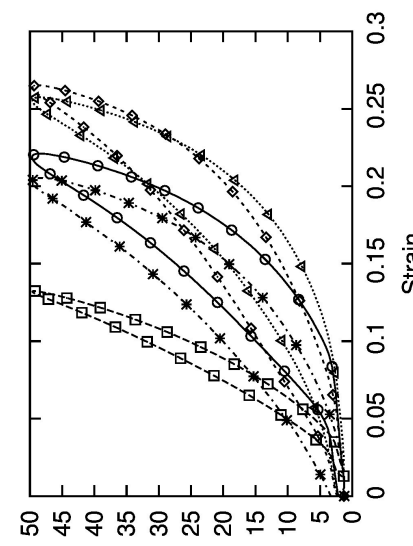

o
:

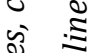

:

ป

o

造 $\frac{\vec{a}}{2}$

ठे

今

:

迥

ป

है

ป ن

岁 $\stackrel{0}{0}$

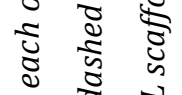

ปั

ป क

ป

ป

Uे

ป ปे ป

ঋ 8 ह

\&

ई

ఏ $\stackrel{\Xi}{\Xi}$

ปั

ป ข

ฐ ฐ

i

ڤ్ట

जी ठे

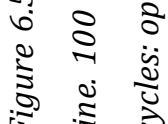




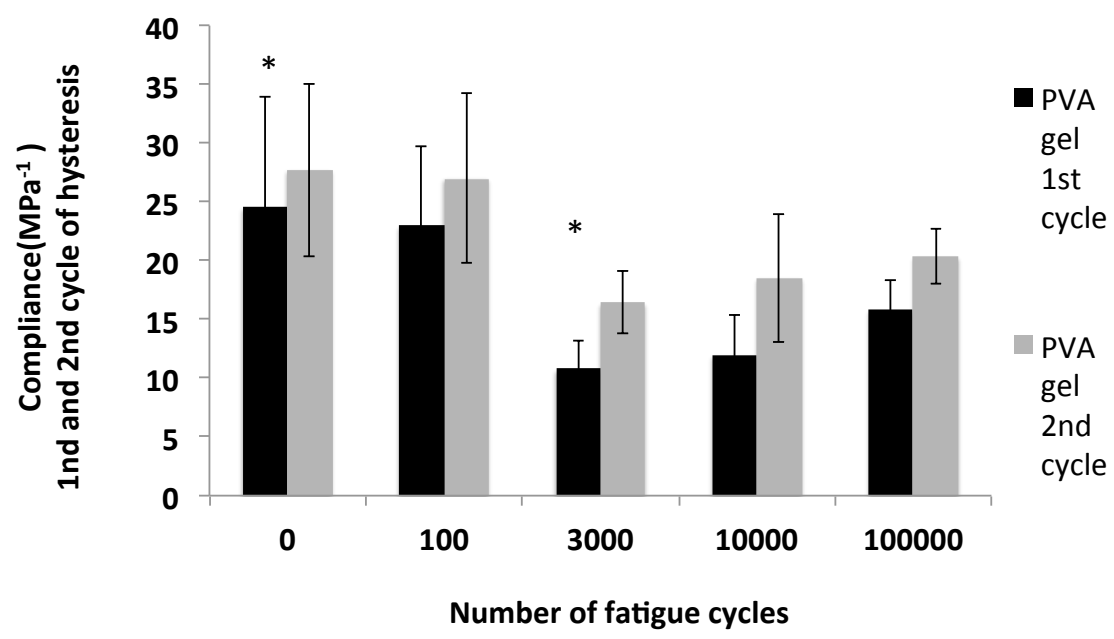

$6.6 a$

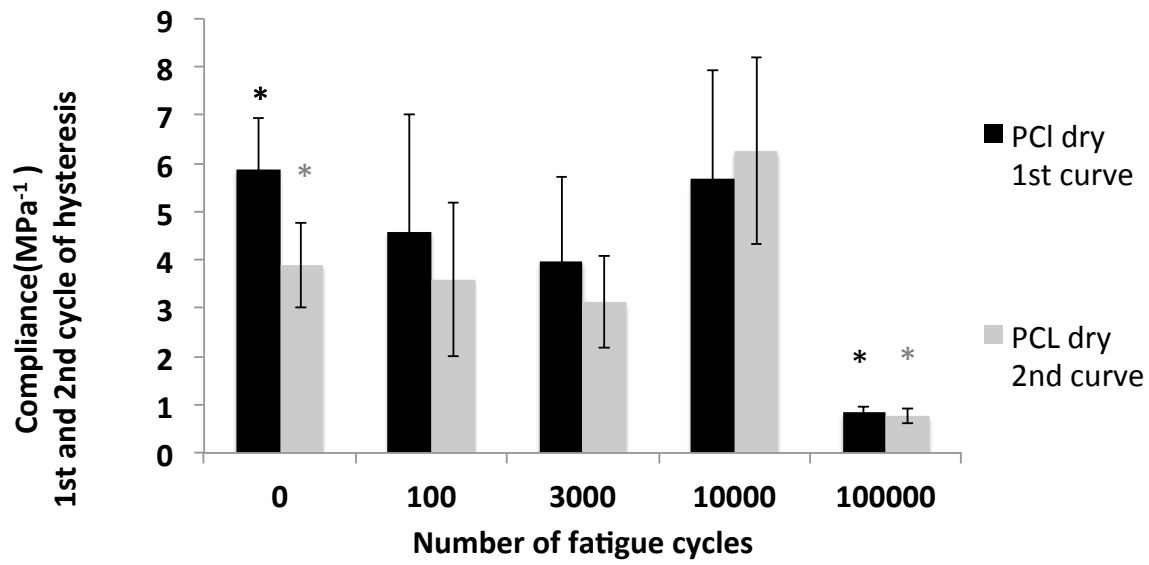

$6.6 a b$ 
An experimental fatigue study of a porous scaffold for the regeneration of articular cartilage
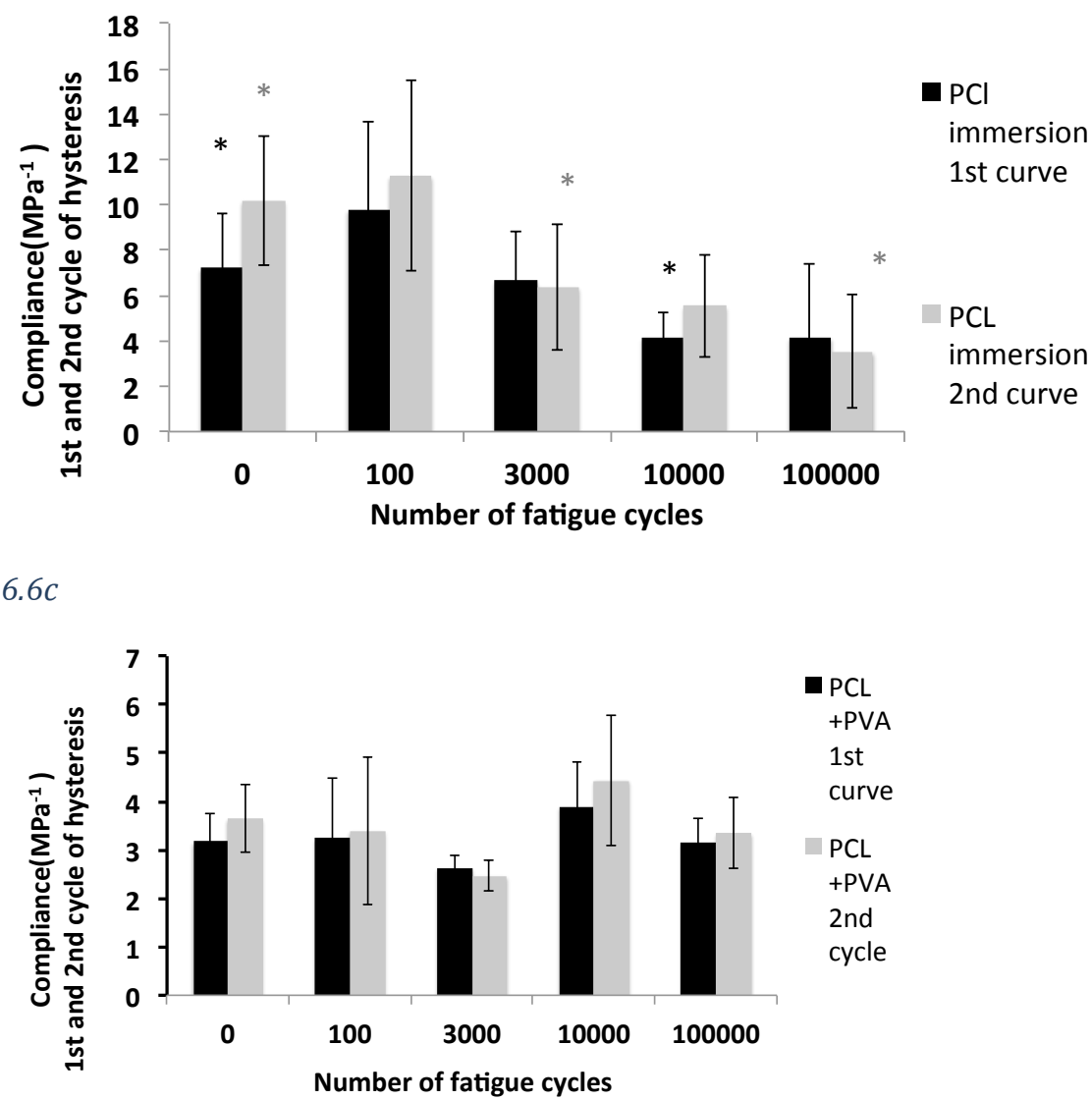

$6.6 d$

Figure 6.6 Compliance (mean value \pm standard deviation) for the first (black) and second (grey) loading curve for the studied systems: (a) PVA gel, (b) dry PCL, (c) immersed PCL, and PCL/PVA (d), as a function of the number of fatigue cycles. ANOVA statistical test was done, and when statistical difference was found an unpaired t-test was performed to evaluate the statistical difference between tested groups with $p<0.05$. Black asterisk is marked for statistical significant difference between the compliance of the first loading curves and grey asterisk for the second loading curve 
The evolution of the compression curves for each time of fatigue (Figure 6.5) balance the observations from the SEM photos. It is seen how the dry and immersed samples show smaller strains after fatigue indicating a more compact material (Figure 6.5b and 6.5c). The PCL/PVA scaffold (Figure 6.5d) has a less prominent strain decrease behavior, proving the effect of the PVA gel inside the pores of the scaffold. The strain for the same load applied is not significantly changed after any of the fatigue times for up to 100,000 cycles proving the stability of the construct.

The results for the compliance give hat the PVA gel (Figure 6.6a) show a quite high compliance for both the first and second loading curve for the samples without fatigue and after 100 cycles of fatigue. There is no statistical difference between the compliance for the first and second loading curves neither throughout the cycles of fatigue, except for the first loading curve at 3000 cycles, indicating stable gels not changing the mechanical behavior for up to 100,000 cycles of fatigue.

The dry PCL samples show no significant difference between the compliance of the first and second loading curves or for increasing cycles up to 10,000 cycles of fatigue (Figure 6.6b). The results have high standard deviation due to the porous nature of the samples. For 
An experimental fatigue study of a porous scaffold for the regeneration of articular cartilage

100,000 cycles the material is now denser, less porous and harder and consequently the compliance is much smaller. The pore damage is irreversible. SEM pictures in Figure 6.1 confirm these results.

The water immersed PCL scaffolds (Figure 6.6c) without fatigue show similar results as the dry samples except the second loading curves that show the effect of water inside the pores. The compliance is increasing in the second loading curve, giving a softer material when the water is expulsed. The dry samples are hardening in the second loading curve, indicating that the material already is denser due to pore buckling. The compliance is slowly decreasing with increasing number of fatigue cycles showing that the samples are affected by the fatigue. After 100,000 cycles of fatigue the material is clearly damaged by the fatigue but in comparison to the dry samples they have been less damaged.

The compliance of the PCL scaffolds with PVA gel inside (Figure 6.6d) do not show any significant change during the fatigue cycles. The hydrogel inside the pores can host a great amount of water, which easily can flow within the scaffold and sustain the loads. 


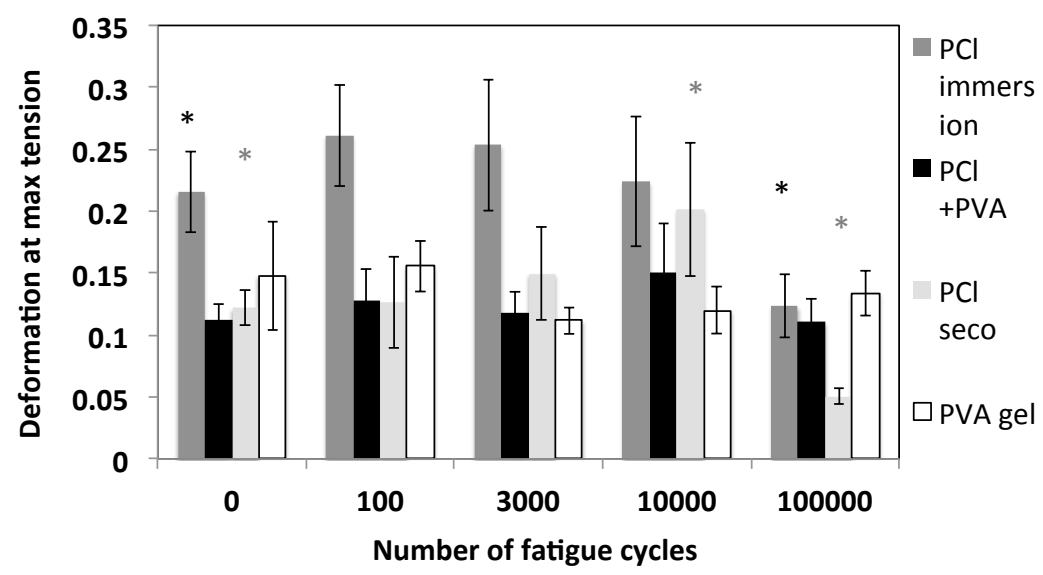

Figure 6.7. Deformation at maximum tension for the studied systems: PVA gel (white), dry PCL (light grey), water immersed PCL (grey), and PCL /PVA (black), as a function of the number of fatigue cycles. ANOVA statistical test was done, and when significant statistical difference was found an unpaired t-test was performed to evaluate the statistical difference between tested groups with $p<0.05$. Black asterisk marc statistical significant difference between PCL immersion groups and grey asterisk between PCL dry groups.

By observing the strain for the maximum tension applied similar results are obtained (Figure 6.7). The dry scaffolds have a strain around $12 \%$ for the samples without fatigue and there is no significant difference up to 10,000 cycles of fatigue, with just a slight increase in the maximum strain. For 10,000 cycles the strain increases to approximately $25 \%$ for the same load. For 100,000 cycles of fatigue the strain diminishes to around 5\%. The material should be very densely packed with little porosity and behaves as a 
An experimental fatigue study of a porous scaffold for the regeneration of articular cartilage

hard material. The SEM images and the values of compliance are in good agreement with these results (Figure 6.1 and 6.6).

The water-immersed scaffolds have an initial strain of about $20 \%$ and then show a slight increase in the strain, indicating some trabeculae bending. After 100,000 cycles the strain is higher than in the dry scaffold, meaning that it has more intact pores. This is confirmed with the SEM images that show the difference of the dry and immersed scaffold after fatigue (Figure 6.1h and 6.1i).

The scaffold filled with PVA gel does not show any significant difference before or between the different cycles of fatigue. The strain is around $12 \%$.

All the samples show hysteresis energy (Figure 6.8). A perfect elastomer does not show any hysteresis curve, the loading and unloading path are identical and no energy is dissipated (248) (249). In previous study (250) a macro and micro porous PCL scaffold was filled with a fibrin gel and mechanically tested for fatigue predictions up to 1000 cycles. The hysteresis energy was calculated and with a mathematical model it was shown that filling the PCL scaffold with water, or fibrin, improved the fatigue life of the scaffold. In this study, for the samples without fatigue 
testing, the dry and immersed scaffolds have a higher plastic strain energy density compared to the scaffolds filled with hydrogel. The PCL/PVA construct shows no significant difference in the dissipated energy throughout the fatigue cycles compared to the samples without fatigue. The dry and immersed PCL scaffolds dissipate less energy after 100,000 cycles of fatigue which again shows a dense material provoked by pores collapse.

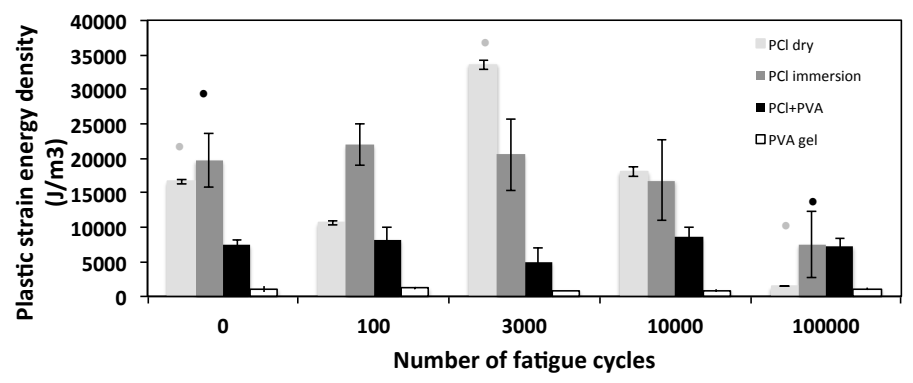

Figure 6.8. The dissipated energy from the second hysteresis cycle from each time of fatigue for the PVA gel (white), dry PCL (light grey), water immersed PCL (grey) and PCL/PVA construct (black). ANOVA statistical test was done, and when significant statistical difference was found an unpaired t-test was performed to evaluate the statistical difference between tested groups with $p<0.05$. Grey asterisk marc statistical significant difference between PCL dry samples and black asterisk between PCL immersion samples. 
An experimental fatigue study of a porous scaffold for the regeneration of articular cartilage

\section{Conclusions}

In this work fatigue tests have been carried out on a micro and macro porous biodegradable Polycaprolactone scaffold in different conditions, dry and immersed state and filled with a PVA hydrogel mimicking the ECM of cartilage. Unconfined compression studies up to 100,000 cycles has been made and the compliance, maximum strain and hysteresis energy were calculated to evaluate the long-term mechanical behavior of this "in vitro" cartilage model. The first and second loading curves of the resulting stress-strain curves have been analyzed for each cycle of fatigue and the morphology has been observed after each fatigue cycle. This gives a comprehensive study of the mechanical behavior of the scaffold in implanted conditions.

The scaffold/gel construct sustains up to 100,000 cycles of fatigue without any alterations in morphology, stressstrain response or hysteresis energy. The scaffold can withstand the compressive force without trabeculae breaking since the water swollen gel inside the pores perceives some of the compressive load. This is crucial in tissue engineering applications where the scaffold is implanted and need to resist the initial physiological 
mechanical stress and offer an adequate biomechanical environment for the cells and the ECM. The results are important when it comes to design and fabrication of porous scaffolds to avoid unnecessary animal trials. In vivo there will be a complex interaction between the cartilage GAGs, the collagen fibers and the water molecules. Water will pass by the ECM components and go through the scaffold trabecular between the pores and to a high extent contribute to the mechanical performance. The results obtained in this study show that the scaffold is able to initially sustain the load applied, and once the cartilaginous tissue grows inside the pores the physiological load applied does not change its mechanical performance. 
The design of a biomedical implant device for articular cartilage regeneration

\section{Chapter 7: The design of a biomedical implant device for articular cartilage regeneration}

This chapter is based on the manuscript: The in vivo response of a biomedical implant device for articular cartilage regeneration, submitted to The International Journal of Artificial Organs in June 2015. As previously stated in the introduction of this thesis, repair of articular cartilage defect is difficult and surgical techniques offered today generate a mostly fibrous cartilaginous tissue. Many tissue engineering strategies currently focus on improving the mechanical properties of the scaffolds, since it is believed that an adequate biomechanical environment induce chondrogenic differentiation of mesenchymal stem cells. Within the objectives in this thesis was the design of a cartilage implant for animal studies. The design is based on a European patented biomedical implant device, with patent number EP201131625 PCT/W02013/178852. The cartilage device is patented by this research group at Universitat Politècnica de València, Dr. Carmen Carda at Universitat de València and Dr. J.C. Monllau at Hospital Santa Creu I Sant Peu. The implant is originally designed for the human articular cartilage, and the task in this thesis was to adapt it to animal studies. The articular 
cartilage surface is approximately $3 \mathrm{~mm}$ thick in humans, nevertheless in animals the cartilage is only $1 \mathrm{~mm}$ thick. In figure 7.1 the original implant design is illustrated. As previously discussed, in regeneration of cartilage the joint must be considered as an osteochondral specimen. Osteochondral implants how ever, suffer from the disadvantage of damaging the subchondral bone to great extent. Alterations of the subchondral bone is seen to provoke tissue repair failure. On the other hand, the scaffold must be able to stay in place in the defect. The defect is normally very irregular and adjacent tissue does not hold the scaffold in place. The anchoring must then be done with as little interventions and surgical steps as possible. Therefore, the patented implant consists of a chondral sponge and a fixation device to the sunchondral bone. The anchodring system is originally (Figure 7.1) assured by a polymeric membrane sited in the scaffold. The objectives within this thesis was to design an implant based on this device, adapted for thinner articular cartilage surface in animals. 
The design of a biomedical implant device for articular cartilage regeneration

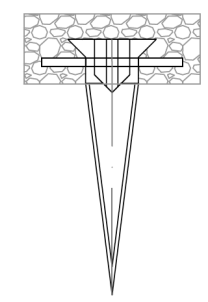

Figure 7.1. The original design for the patent EP201131625 PCT/WO2013/178852. (157)

Two animal studies have been carried out: In the first study a $15 \%$ PCL scaffold was implanted in a microfracture defect in 21 sheep. In the second trial the device was improved with the subchondral bone anchoring part, based on the patented implant. The scaffold was synthetized from a $25 \%$ PCL solution and less porogen ratio, to improve the mechanical properties of the scaffold. The scaffold was fixed to a commercially available PLLA pin as anchoring system to the subchondral bone. The scaffold device was implanted in a microfracture defect in 6 sheep. 


\section{Results and discussion}

In the first trial a PCL macro and micro porous scaffold was employed. The scaffold was synthesized by freeze extraction method as previously described in the thesis. Scaffolds were cut with circular stamps and surgical scalpels to cylinders of $4 \mathrm{~mm}$ diameter and $1 \mathrm{~mm}$ height.

For the second trial the PCL scaffold was fixed to the subchondral bone using a combination of the PCL scaffold and a biocompatible pin as shown in Figure 7.2. Due to the difference in cartilage thickness of humans and animals, the anchoring ring did not fit into the porous scaffold and had to be placed underneath the scaffold.

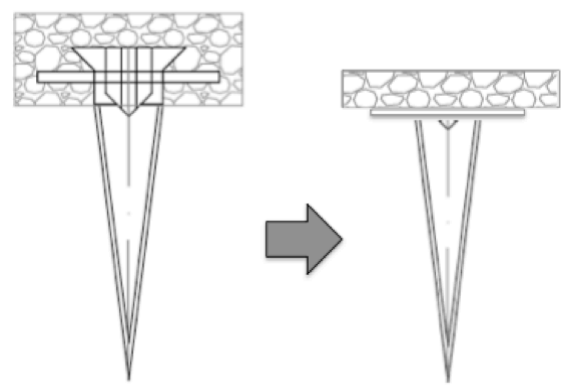

Figure 7.2. The left picture show the original implant device, and the right picture show the modified implant for the animal studies. It can be observed that the porous sponge not contain the polymeric membrane anymore, and that the fixation device is attached at the underlying bottom of the implant. 
The design of a biomedical implant device for articular cartilage regeneration

In the device implanted in the sheep, the anchoring system consisted in a commercially available Poly(L-lactic), PLLA, pin (Contour ${ }^{\mathrm{TM}}$ Meniscus Arrow ${ }^{\mathrm{TM}}$, ConMed, USA). The PCL scaffold was synthesized from a 25\% PCL solution and 1:1 PCL solvent/porogen spheres ratio. This composition was chosen to increase the mechanical strength of the scaffolds in comparison to the scaffold in the first trials. The PLLA pin is normally used for meniscus operations and specially purchased since it has proven biocompatibility and boneintegration properties in clinics. The PLA pin was attached to the PCL solution in the synthesizing step. The head of the arrow was melted (IN2100 Quick Soldering Iron, JBC, Spain) to have a flat end. The PCL solution and PEMA/PMMA microspheres were rapidly mixed and inserted into a custom made Teflon device of $1 \mathrm{~mm}$ depth and $4 \mathrm{~mm}$ diameter. The PLLA arrow was placed on top of the polymer mix, and the Teflon device was immersed in liquid nitrogen. The freeze extraction and porogen leaching were performed as previously described. The scaffolds were dried in continuous vacuum extraction until constant weight, and placed individually in Eppendorf tubes and sealed in vacuum pockets. The material was sent to sterilization with gamma rays 25 kGys (Arragogamma, Spain). 


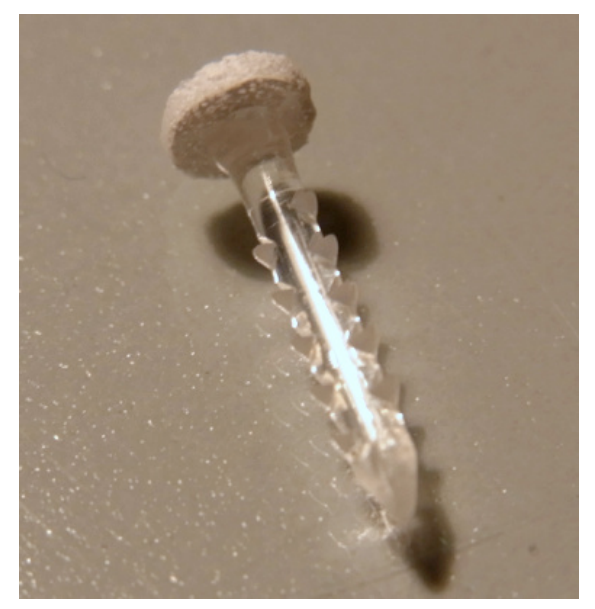

Figure 7.2. The implant device for the second animal trials. The chondral part consists of a porous PCL scaffold and is attached to a PLLA arrow, as anchoring system to the subchondral bone.

In total, an amount of 21+6 ( Trial 1 and 2) mature female sheep, breed Merina and official brand 270BK (Supplier Agricola Ramadera Les Anglades Vilert, Girona, Spain), were employed. The sheep weighed about $40 \mathrm{~kg}$. The surgical interventions was carried out by by Dr. Santos Martinez Diaz at the Hospital Clínic Veterinari at the Universitat Autònoma de Barcelona. The animal care before, under and after the operations was taken care of Dr. Felix García and his staff at the departament de Medicina i Cirurgia Animals. The trials were realized in accordance to protocol CEEA 1940 17-02-2013 approved by the Ethic Committee for animal trials at the Universitat Autònoma de Barcelona. The protocol follows the 
The design of a biomedical implant device for articular cartilage regeneration

European regulations 86/609/EEC (European Economic Community) and the law 5/1995 with regulatory decision $1201 / 2005$ by the local government. After surgery the animals spent a postoperative period in boxes and were released after seven days in conventional herd at the Universitat Autònoma de Barcelona, named "Servei de Granges I Camps Experimentals" number B9900042. The animals were divided into three groups. The first experimental group with 15 animals underwent surgery with 2 chondral lesions in the load bearing zone in the interior medial condyle of femur. 4-5 microfractures were done in the defect, in the subchondral bone with an awl for arthroscopic surgery. The PCL scaffold was implanted. The second group, with 6 animals, underwent the same surgery without posterior scaffold implantation. The third group of 6 animals had the same surgery, with the addition of an orifice in the middle of the chondral lesion performed by a Kirschner needle. The orifice was made to facilitate the implantation of the PCL scaffold with PLLA pin. One of the animals perished during the postoperative procedure due to anesthesia problems.

The sheep were anesthetized by tracheal intubation and laid in supine position. Surgical procedure was performed on the right knee as described below. Sterile field was 
prepared following standard procedures, first the right knee was shaved and the surgical site was disinfected using iodine solution and animal non-sterile zone was covered with sterile towels. A $40 \mathrm{~mm}$ incision was performed in the medial knee in distal position to patella. An internal distal arthrotomy was performed in the lower part of the patella in the medial height of patellar tendon, separating sidelong the patellar ligament and the patella, without dislocating, and separating the internal structures of the joint capsule allowing access to the load bearing zone of the internal condyle of femur.

Then, 2 chondral lesions of $4 \mathrm{~mm}$ diameter and approximately $1 \mathrm{~mm}$ height in weight bearing zone of the internal femoral condyle was performed, with 4-5 microfractures until bleeding from subchondral bone. To perform the microfractures, an Arthrex awl used in human microfracture surgery was employed. The defect was washed with physiologic serum. In the group one, a PCL scaffold was implanted by press-fit technique (Figure 7.3). The control group was left with microfracture without scaffold implantation. In trial two, the six animals had an additional orifice made in the center of the chondral defect. The scaffolds were placed in the chondral defect with sterile tweezers and press-fit technique (Figure 7.4). 
The design of a biomedical implant device for articular cartilage regeneration

In all groups the arthrotomy was closed with loose vycril sutures $3 / 0$, and the skin with continuous vycril sutures 4/0 and loose paper (Steri-Strip 3M) sutures. The lesion was covered with standard bandage (Sterile Cutiplast 7.2 $\mathrm{cm}$ x $5 \mathrm{~cm}$, Smith\&Nephew). After the surgery and removal of the anesthesia the animals were returned to cages, wearing soft knee bandage in flexion position for 7 days. The animals were standing up about half an hour after operation. After 7 days the animals were released in controlled herds according to the norms approved by CEEA.

The sheep were scarified after 4,5 months free movement in the herd. The animals were first placed in boxes and euthanized by intravenous overdose of pentobarbital 100 $\mathrm{mg} / \mathrm{kg}$. Once euthanized the samples were obtained for posterior analysis. The samples were obtained by dissection of the right knee and exposure of the internal femoral condyle, by osteotomy with an oscillating saw. 


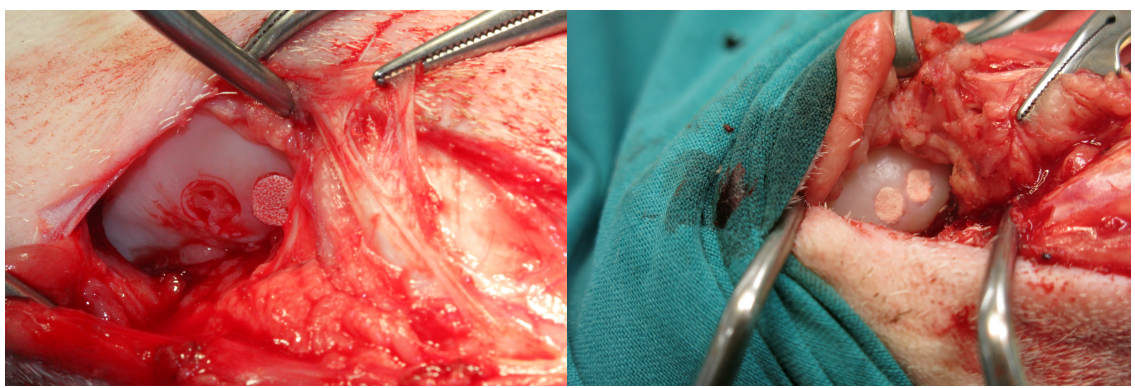

a)

b)

Figure 7.3. The surgery for trial 1. A) Two lesions of 4mm diameter were created in the medial condyle of femur and a microfracture was performed in the defects. B) In each of the lesions a PCL scaffold was placed with press-fit technique.

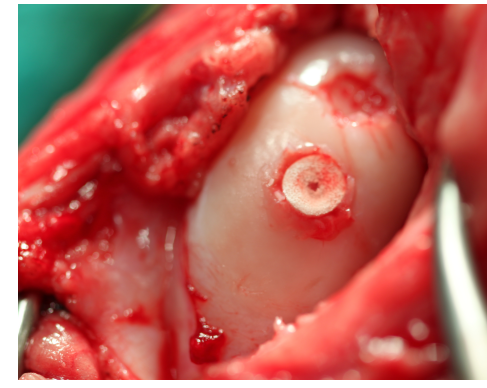

a)

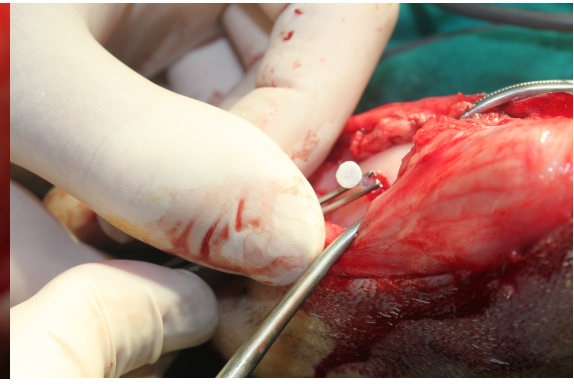

b)

Figure 7.4. The surgery for trial 2. Two lesions of $4 \mathrm{~mm}$ diameter were created in the medial condyle of femur and microfracture was performed in the defects. In each of the lesions the PCL scaffold with PLLA screw was placed with press-fit technique. 
The design of a biomedical implant device for articular cartilage regeneration

The internal femoral condyle of the left knee was also obtained, to use as control. The samples used for biomechanical testing were sent in sterile PBS buffer medium with penicillin/streptomycin 1ml/100 ml PBS with $0.5 \%$ azide. The temperature was controlled to $4{ }^{\circ} \mathrm{C}$ from explant before mechanical testing. After the mechanical testing, the samples were fixed in formalin solution $10 \%$ neutral buffer for 48 hours. Then the samples were washed and saved in PBS+azide.

For the biomechanical tests, the hole cartilage piece with the subchondral bone was subjected to indentation tests, previously described. The results can be seen in figure 7.8. The elastic modulus of the joints with scaffold implantation are inferior to controls in most cases, supported by the histology analysis.

\begin{tabular}{|l|l|l|l|}
\hline Sample & Surgery & Examination & $\begin{array}{l}\text { Time } \\
\text { (months) }\end{array}$ \\
\hline 1 & Microfracture & Histology & 4.5 \\
\hline 2 & Microfracture+scaffold & Histology & 4.5 \\
\hline 3 & Microfracture & Histology & 4.5 \\
\hline 4 & Microfracture+scaffold & Histology & 4.5 \\
\hline
\end{tabular}




\begin{tabular}{|l|l|l|l|}
\hline 5 & Microfracture+scaffold & Histology & 4.5 \\
\hline 6 & Microfracture+scaffold & Histology & 4.5 \\
\hline 7 & Microfracture & Histology & 4.5 \\
\hline 8 & Microfracture+scaffold & Biomechanical & 4.5 \\
\hline 9 & Microfracture+scaffold & Biomechanical & 4.5 \\
\hline 10 & Microfracture+scaffold & Biomechanical & 4.5 \\
\hline 11 & Microfracture+scaffold & Histology & 9 \\
\hline 12 & Microfracture+scaffold & Histology & 9 \\
\hline 13 & Microfracture+scaffold & Biomechanical & 9 \\
\hline 14 & Microfracture+scaffold & Histology & 9 \\
\hline 15 & Microfracture+scaffold & Histology & 9 \\
\hline 16 & Microfracture & Histology & 9 \\
\hline 17 & Microfracture & Histology & 9 \\
\hline 18 & Microfracture+scaffold & Biomechanical & 9 \\
\hline 19 & Microfracture & Histology & 9 \\
\hline 20 & Microfracture+scaffold & Biomechanical & 9 \\
\hline
\end{tabular}

Table 7.1. References for each sample from trial one. For each sample the internal femoral condyle of left knee was used as control. 
The design of a biomedical implant device for articular cartilage regeneration

\begin{tabular}{|l|l|l|l|}
\hline Sample & Surgery & Examination & Time(Months) \\
\hline 1 & Microfracture+device & Histology & 4.5 \\
\hline 2 & Microfracture+Device & Histology & 4.5 \\
\hline 3 & Microfracture+Device & Histology & 4.5 \\
\hline 4 & Microfracture+Device & Histology & 4.5 \\
\hline 5 & Microfracture+Device & Histology & 4.5 \\
\hline 6 & Microfracture+Device & Histology & 4.5 \\
\hline
\end{tabular}

Table 7.2. References for each sample from trial 2. All 6 animals had microfracture+ PCL scaffold+PLLA pin device implantation.

The histological analysis and staining was performed at the Facultat de Medicina i Odontologia at the Universitat de València under charge of Dr. Carmen Carda and Maria Sancho Tello. Macroscopic pictures of the femoral condyles were taken using a Leica DC150 camera. Morphology was studied following standard histological technique. Briefly, after fixation of the joint specimen, the samples were rinsed with PBS and immersed in Osteosoft decalcifier solution (Merck) up to 4 months at room temperature. Once specimens were decalcified, they were cut through the middle of the scaffold and each half was 
separately embedded in paraffin, following standard histological proceduces. Five- $\mu \mathrm{m}$ thick sections were obtained and stained with hematoxylin-eosin and Masson's trichrome. Stained sections were analyzed under Leica optical microscope (Leica DM 4000B), and pictures were taken using a Leica DFC 420 camera. The samples obtained in the second trial followed the same procedure except they were embedded in low-melting embedding wax instead to standard paraffin, in order to preserve the implanted scaffold.

Macroscopic study of the articular surface at the injury zones shows the appearance of neotissue covering partially or completely the injury zone, although the surface had a rougher appearance than controls (Images not presented). No differences were found in the macroscopic study in the different trials. However, when samples were sectioned into halves, big cystic cativities were observed in many samples of the first trial, similarly to those observed in other studies using large mammals. The presence of cystic cavities in the second trial was less frequent, the cavities were smaller and located at the lower end of the anchoring pin.

Microscopic study revealed (Figure 7.6-7.9) that all samples presented an active process of neotissue 
The design of a biomedical implant device for articular cartilage regeneration

formation around the edges of the injury, as previously seen in smaller mammals. In some samples, neotissue covered completely the articular surface although the thickness was less than in controls) but in others the neotissue only covered partially the biomaterial, which was in contact to sinovial cavity, in both trials studied. Figure 7.6 and 7.8 represent typical panoramic results from both groups.

In the first group (only PCL scaffold), the repaired tissue showed the appearance of hyaliane-like cartilage. Microscopic analysis (Figure 7.6) revealed that it was mainly immature hyaline cartilage, with a higher cellular density than controls with only microfracture surgery. The cells did not present the arrangement of the mature hyaline cartilage at the time of sacrifice (Figure 7.7a-b). The neotissue covering the injury zone had also areas of fibrous tissue. Cystic cavities were big and abundant in the first trial, and they contained either an immature connective tissue (Figure 7.7c), of they were empty (not shown).

In the second group, where the PCL scaffold was attached to a PLLA anchoring pin, microscopic study revealed they also presented an active process of repair in the injured surface as well as around the pin (Figure 7.8). Similar to 
first trial group, the neotissue found on the articular surface was mainly immature hyaline-like cartilage (Figure 7.9a), and areas of preserved scaffold could be observed (Figure 7.9b ). In the subchondral bone, the area occupied by the anchoring pin appeared as an empty rectangular cavity, since the PLLA pin was not preserved, and was surrounded mainly by fibrous connective tissue containing big multinucleated cells with a phagocityc aspect (Figure 7.9c). Some areas also contained loose or mesenchyme connective tissue around the pin cavity. In this group, none or small cystic cavities were observed (16\% of the samples), that were around the deeper end of the anchoring pin. It can be seen that the improved scaffold with fixation system to subchondral bone shows better tissue integration. The articular surface shows an active reparative process, whereas subchondral bone shows less alterations with reduced cyst formation. It seems like that the presence of the PLLA pin enhance the biointegration, and prevents cyst formation. The macroscopic study show that most of the defects, $75 \%$, exhibited the articular surface completely or almost completely with a repair neotissue. The macroscopic aspect was similar to normal cartilage, although the appearance was rougher than controls. Regarding the fixation system, the union between the PCL scaffold and 
The design of a biomedical implant device for articular cartilage regeneration

PLLA pin needs to be improved since many defects macroscopically show that the scaffold had been lost.

Both groups of scaffold implantation showed an increase in surface thickness. These differences were statistically significant when compared normal cartilage with treated groups. The percentage of regenerated cartilage was 68.7 $\%$ in the PCL scaffold group and $53 \%$ in the microfracture group, which was a difference statistically significant ( $p$ $<0.05)$.

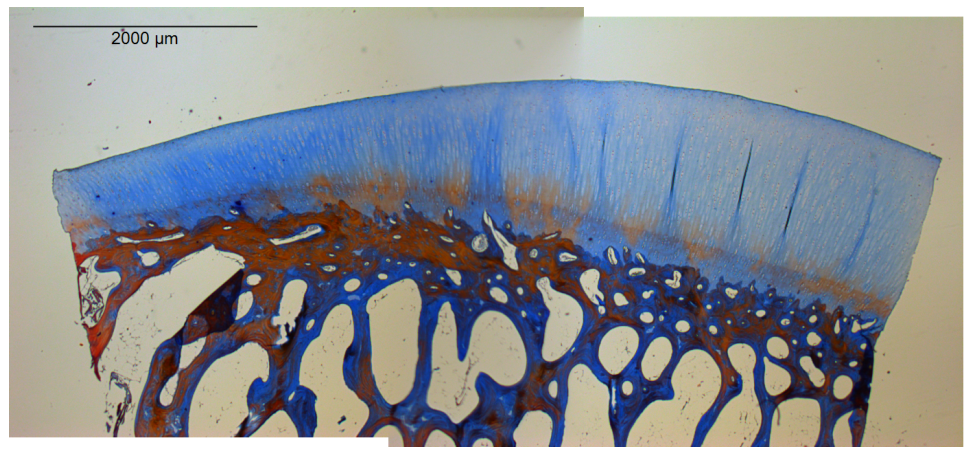

Figure 7.5. The contralateral knee after 4.5 months implantation, serving as control. 


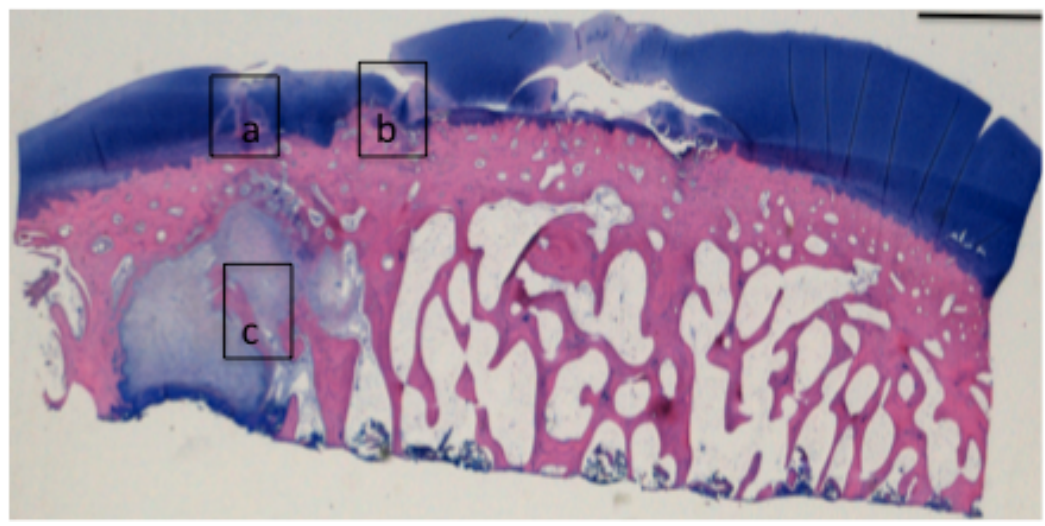

Figure 7.6. Panoramic view of a sample after 4.5 month implantation of PCL scaffold and microfracture surgery. The two defects are seen partially filled with a neotissue and the subchondral bone is showing alterations with cysts formation. Scale bar $1 \mathrm{~mm}$.

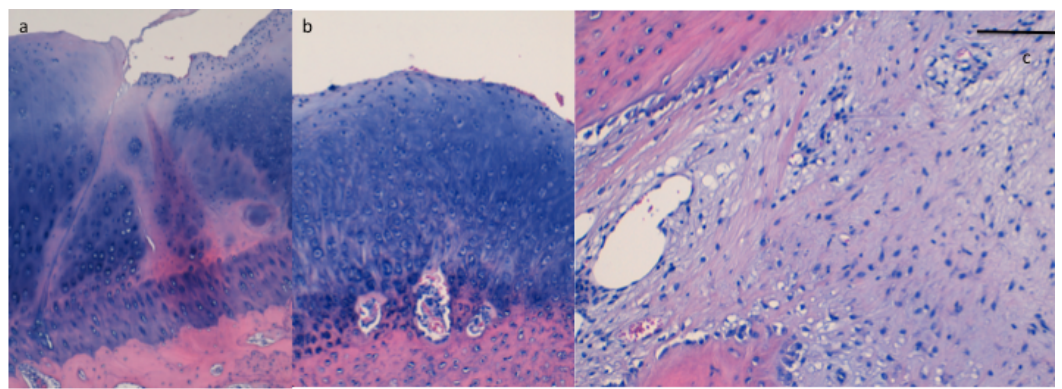

Figure 7.7. Microscopic images of the sample shown in Figure 7.6. A) limit between native cartilage and neotissue b) neocartilage growth at the defect site and c) cyst formation in the subchondral bone. Scale bar $1 \mathrm{~mm}$. 
The design of a biomedical implant device for articular cartilage regeneration

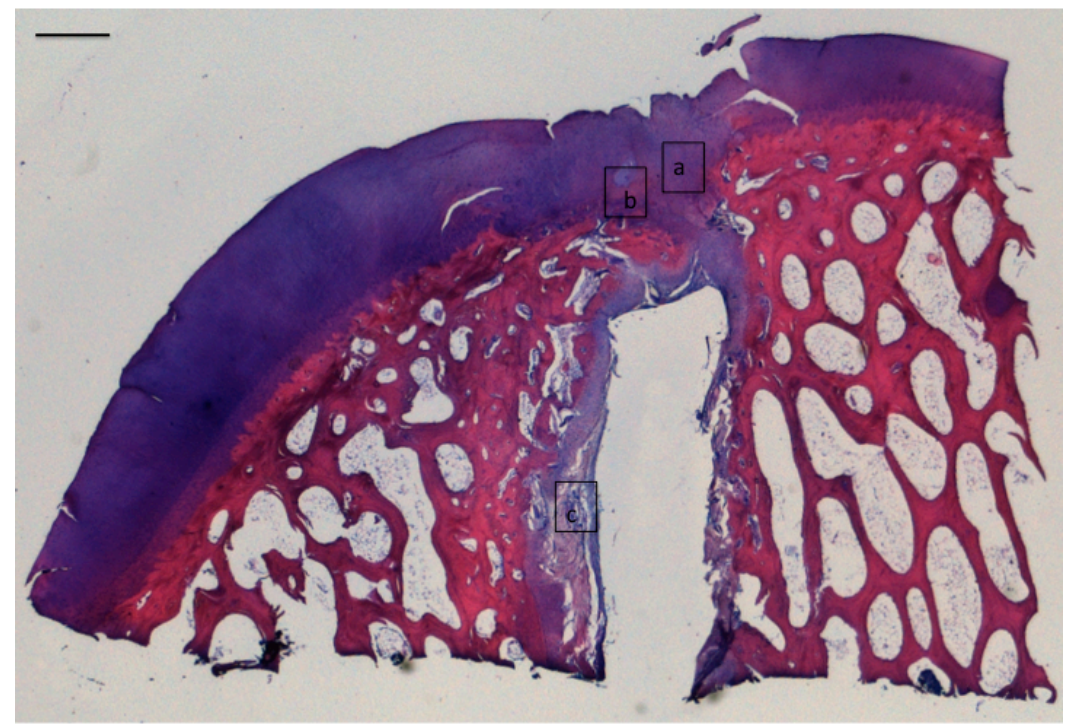

Figure 7.8 Panoramic view of a sample after 4.5 months implantation of PCL scaffold with subchondral bone anchoring after microfracture surgery. The defect has been filled with a neotissue, and subchondral bone lacks alterations. Scale bar $1 \mathrm{~mm}$.

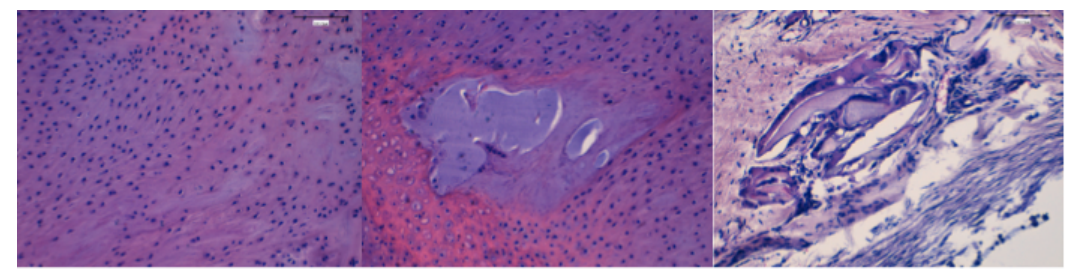

Figure 7.9 Microscopic images of the sample shown in Figure 7.8. A) Regenerated neotissue is immature cartilaginous tissue b) PCL scaffold, degraded or broked into smaller pieces, surrounded by regenerated neotissue c) Remains of PLLA pin degraded by multinucleated phagocitic cells. Scale bar $1 \mathrm{~mm}$. 


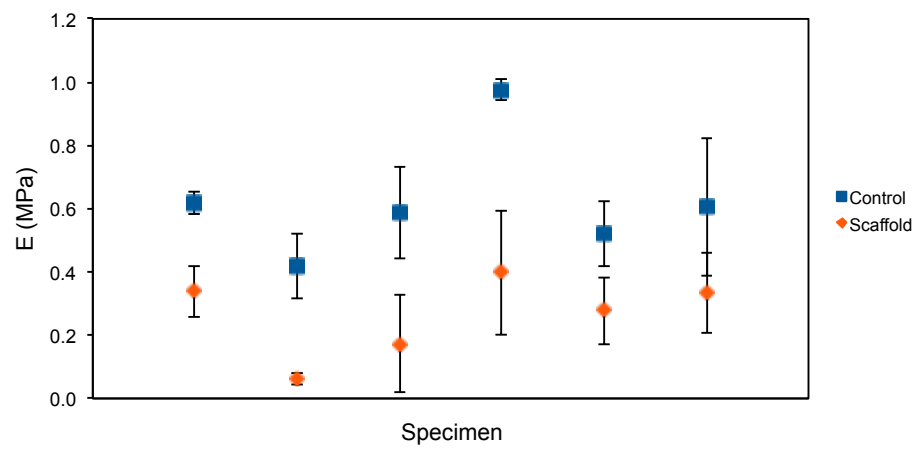

Figure 7.8 The results from the indentation tests of the cartilage and bone piece after sacrifice in the first trials with implantation of PCL scaffold after microfracture surgery. The red points show the specimens with PCL scaffold implantation and the blue points the contralateral healthy knee. The results show that the repair tissue has inferior mechanical modulus than controls.

\section{Conclusions}

Regarding the implant consisting of PCL scaffold with subchondral bone anchoring, the macroscopic study showed that most of the defects (75\%) exhibited the articular surface completely or almost completely repaired with a neotissue. Nevertheless, they had a rougher appearance than controls. Besides, only 2 samples (16\%) presented a cystic cavity, which was in the deeper end of 
the pin. These results showed an improvement from the defects where only PCL scaffold was implanted without anchoring pin, that resulted in a high incidence of cystic cavities. Histological study reveals an active repair process in the injured surface as well as around the pin. All samples presented neocartilage formation at the edges of the injury, that eventually covered the whole surface in some cases, although the cartilage observed had an immature aspect at the time of study most of the times. Around the pin, fibrous, loose or mesenchymal connective tissues were observed along with an active process of primary ossification. Subchondral bone shows an active process of bone formation around the pin, and it presents a reduced formation of cystic cavities. Therefore, the device implanted improves tissue regeneration in large mammals, although it needs to be optimized 


\section{Discussion}

A PCL scaffold with different micro porosity, but maintaining the same macro pores has been synthesized and a PVA hydrogel has been introduced into the pores of the scaffold. The PCL/PVA construct has been subjected to different cycles of freezing and thawing. The PVA hydrogel itself has also been characterized. It can be observed by SEM photos (Figure 5.1), that the PCL scaffold shows a double interconnected porous structure with macro-pores ranging from approximately $120 \mu \mathrm{m}$ to $200 \mu \mathrm{m}$ and micropores up to $10 \mu \mathrm{m}$. The macro-pores are produced by the porogen leaching process and the micro-pores result from the extraction of dioxane crystals formed in the freezing process. Thus, an increase in the PCL/dioxane ratio decreases the micro-pores size. Scaffolds with 3 different concentrations of PCL were fabricated (15/20/25\% PCL) and the micro-porosity is clearly observed in the samples for 15\% PCL (Figure 3.1). However, by increasing the PCL content the micro-pores diminish and can hardly be distinguished for high concentration of PCL (Figure 5.1). The micro-pores appear more like a rough surface in the scaffold walls for the 25\% PCL (Figure 5.1). It seems like there is a limit for the concentration of PCL for the creation of micro-pores. The polymer concentration 
influences the micro-pore size by being inversely proportional to the crystals formed during the freezing step. Higher polymer concentration means fewer and probably smaller crystals, that will results in smaller micro-pores, since every micro-pore consists of groups of solvents crystals.

By porosity measurements and SEM images it is clear that the PVA hydrogel enters all micro and macro pores of the PCL scaffold (Figure 3.1). The hydrogel appears in a honeycomb pattern, covering all pores in the PCL scaffold. In previous studies by other authors morphological changes of the PVA hydrogels after freezing and thawing have been reported for different concentration of polymer (167). It was also noticed that the gel with just one or two cycles of freezing and thawing did not appear porous due to polymer collapse during preparation steps. Regarding any morphological change in pore size for the different cycles of freezing and thawing, different results were reported, some authors seeing a pore growth for increasing cycles of freezing and thawing, and others none. (184) (185) (168) The pore size for three cycles of freezing and thawing is reported to be around $1 \mu \mathrm{m}$. (168) The porous structure observed in the PVA hydrogels is stated to arise from the cycles of freezing and thawing 
since the ice crystals form the pores. We did not observe any difference in pore size between 1 and 3 cycles of freezing and thawing but an increase in pore size was seen for the hydrogel after 6 cycles of freezing and thawing up to approximately $10 \mu \mathrm{m}$ (Figure $5.2 \mathrm{e}$-f). This phenomenon was seen both for the pure hydrogel and for the hydrogel inside the PCL scaffold. Inside the scaffolds pores the hydrogel pores appear larger and this could be due to the PCL limiting the polymer rich phase growth during freezing and thawing (Figure $5.2 \mathrm{a}-\mathrm{c}$ ). Regarding the shape of the pores, it is distinguished a linear direction of the pores, possibly due to the temperature gradient during freezing, or sublimation in the cryo-SEM unit.

Fatigue studies were carried out on the PCL scaffolds in dry, water immersed and hydrogel filled conditions. After each fatigue time the morphology was observed to evaluate the performance of the scaffold. It could be observed that the dry and water immersed scaffolds did not suffer any morphological change due to fatigue until 10,000 cycles (Figure $6.1 \mathrm{f}-\mathrm{g}$ ). The pores in the dry samples were then collapsed and the scaffold suffered an overall structural change due to fatigue wear. The water immersed scaffolds suffered less morphological changes, the scaffold trabeculae did not break yet. After 100,000 
cycles of fatigue the dry scaffold was totally collapsed and the water immersed scaffold maintains a porous structure (Figure $6.1 \mathrm{~h}-\mathrm{i}$ ). This shows the effect of water inside the pores of the scaffold. The potentially plasticizing effect of water to the polymer chain is not seen, at the contrary water inside the scaffold pores increase the resistance to compressive force during fatigue. This can be understood by considering water as an incompressible liquid and take in account the relative high compressive velocity the experiments were performed, $1 \mathrm{~mm} / \mathrm{min}$. The water is not given time to leave the scaffold pores and hence prevents the scaffolds pores from collapse during fatigue. The morphology of the hydrogel filled scaffolds is not seen affected by fatigue (Figure 6.2). The scaffolds sustain fatigue without undergoing any morphological changes, by having a water-filled highly crosslinked gel inside the pores. The possible effect of freezing on the scaffolds of PCL is neglected since it is believed to affect the morphology very little.

Scaffolds of P(EA-HEA) with different crosslinking density show an interconnected spherical porous structure. The scaffold pores are more open and interconnected for increasing crosslinking density, due to the synthesize process (Figure $4.1 \mathrm{a}-\mathrm{c}$ ). After compression tests the P(EA- 
HEA) scaffolds show a slight superficial morphological change. The SEM photos of the hydrogel filled scaffolds show that the gel is filling all pores, before and after compression tests (Figure $4.4 \mathrm{~d}$-f). After compression the hydrogel appears lightly compressed inside the scaffold, probably due to scaffold elasticity.

In chapter 3 the mechanical properties of 15\% PCL scaffold filled with PVA hydrogel for different cycles of freezing and thawing was evaluated. The water immersed PCL scaffold shows typical stress-strain behavior for porous materials, with linear-elastic region, pore collapse zone and densification region (Figure 3.3). Dry and immersed scaffolds show similar apparent elastic modulus, indicating that the effect of water inside the scaffolds pores is seen foremost as a fatigue resistance effect (Figure 3.5). This will be discussed further regarding the mechanical properties after fatigue tests. The study presented in chapter 3 indented to obtain an experimental model simulating the mechanical behavior of a porous scaffold implanted in a cartilage defect. In a cartilage defect the mechanical properties of the scaffold would change with time, since cells, ECM and finally cartilaginous tissue would fill the pores. The PVA hydrogel was chosen as cartilage model for the high water content, mechanical 
properties and porous structure of the hydrogel. Mechanical properties of the PVA hydrogel were easily tailored by different cycles of freezing and thawing. The compressive modulus increased with the number of freezing and thawing cycles (Figure 3.4), owed the increased crystalline regions that serve as crosslinks. The stress-strain behavior of the hydrogel filled scaffold after six cycles of freezing and thawing clearly showed the influence of PVA (Figure 3.3 and 3.5). The hydrogel prevented pore collapse, and the PCL/PVA further more showed a synergic effect resulting from the water filled hydrogel inside scaffold pores. The water inside the hydrogel and the scaffold pores will result in interesting features: The mechanical modulus decrease in the PCL/PVA when the PVA is lightly crosslinked (Figure 3.5). This is believed to be due to water permeability through the PVA hydrogel, and consequently through the PCL/PVA scaffolds. If the water expulsion is dependent on the crosslinking density of the hydrogel, water will flow out easier from the soft hydrogel and result in lower elastic modulus. The same pattern was seen for the P(EA-HEA) scaffold filled with PVA. The modulus decreased for the hydrogel filled scaffolds, implying easier water expulsion (Figure 4.2). Therefore, permeability tests were performed for the PVA hydrogels and the PCL/PVA scaffolds. (Figure 
5.3) By confined compression studies the permeability of the scaffolds and gels was deduced. Permeability for PVA hydrogels was highly dependent on the cycles of freezing and thawing (Figure 5.4a). This can easily be understood by imagine the viscous solution of PVA, the hydrogel after one cycle of freezing and thawing is nothing but a lightly crosslinked solution. After six cycles of freezing and thawing PVA is yet a stiff hydrogel with hard water passage. Curiously enough permeability for the PCL/PVA scaffolds does not change with the number of freezing and thawing cycles (Figure 5.4a). This was only seen in the pure PVA hydrogels and not in the PCL/PVA constructs. Confined compression tests on PCL/PVA scaffolds with different micro porosity was done and it was concluded that the micro porosity had more influence on the permeability than the hydrogel filling (Figure 5.4b). Permeability and aggregate modulus values of the scaffold with hydrogel were close to human cartilage. There was no significant statistical difference in permeability between the same kind of scaffold water filled or filled with hydrogel (Figure 5.4 b-c). That means that water is giving the same resistance to flow through a hydrogel filled scaffold, as a water filled scaffold. The hydrogel contains $90 \%$ water and it seems like the crosslinks do not hinder the water flux through the scaffold. Linking this to 
the results in chapter 1 , where it was seen a decrease in the mechanical modulus with a soft hydrogel filling compared to water filled scaffold, gives a new perspective. The dense hydrogel is increasing the mechanical properties of the PCL scaffold, compared to the water filled samples. Albeit, the hydrogel filled scaffold have same values of permeability as for the water immersed scaffold. Additionally the soft hydrogel, with less crosslinking points, soften the scaffold when introduced into the pores.

It seems like there is a more complex relation between the decrease in modulus, water expulsion and water permeability. This could be due to the fact that permeability was calculated through confined compression studies that are based on the biphasic model of cartilage (40) (41). In fact, the PCL/PVA system consists of three systems. The PCL scaffold is one phase, and the polymeric chains of PVA is another phase and water is a third phase. The biphasic system takes in account two phases, polymeric chains and water. For the PVA hydrogels the model is accurate, it is seen how permeability increases for decreasing crosslinking density. (Figure 5.4a) This could explain why the elastic modulus is decreasing in the low crosslinked scaffold/hydrogel construct without affecting permeability values (Figure 
3.4) The water expulsion is facilitated but not reflected in permeability experiments. Hydraulic permeability studies with bioreactor should complete the study to bring more light on the phenomena.

It is however seen that the aggregate modulus for the PCL/PVA construct for 1-6 number of $\mathrm{f} / \mathrm{t}$ cycles is without significant differences throughout all the cycles. (Figure 5.6) The aggregate modulus is, as said, the modulus in equilibrium when the water flow ceased, calculated from the confined compression tests. It can be seen that the aggregate modulus and the permeability results obviously agree since the former is calculated from the latter. The Young's modulus is calculated from unconfined compression tests and might not be the right value to compare to the permeability in this case.

It might be interesting to compare these findings with results from others. In studies with agar in aqueous solutions, motion of water molecules are unaffected by the presence of agar molecules. (185) This means that water passes by the agar molecules without being influenced, somewhat similar to water motion inside the PVA gel in the PCL scaffold. In another study, a porous Poly(1.8octanediol-co-citric acid) scaffold was tested in a flow chamber, with different porosities filled with Hya/Col I 
hydrogel. (251) It was seen that the permeability was not affected by the increased porosity, when hydrogel filled. When the scaffold was filled with water, the permeability increased for increased porosity but in the hydrogel filled the permeability was constant. This is interesting since it shows that water motion through scaffolds with hydrogel inside, is independent on the internal scaffold structure. In the case with agar, water motion was unaffected by polymer chains and in the case of POC scaffold filled with hydrogel permeability was unchanged for different porosities. The latter is contradicting the results in this thesis, where an increased micro-porosity decreased permeability. It is clear that more experiments are needed to totally understand the water flow phenomena.

The scaffolds fatigue resistance was measured as mechanical modulus after different times of fatigue cycles. The PCL scaffold was tested in dry, water immersed and PVA filled conditions. The stress-strain behavior of the scaffolds was evaluated after fatigue test. The PVA hydrogel showed an almost perfect elastic behavior, before and after fatigue tests. When first and second loading curve was compared for any dry or water immersed scaffolds, it could be seen that the first and second loading curve before fatigue differ from between 
each other. However, after fatigue the first and second loading curve was coinciding, meaning that the material was compact (Figure $6.4 \mathrm{~b}-\mathrm{c}$ ). The compliance was decreasing for increasing fatigue times (Figure $6.6 \mathrm{~b}-\mathrm{c}$ ). Stress softening or stress hardening effects have been pointed to explain the difference between the first and second loading curve. The sample presents a higher elastic modulus after subjected to the first measuring scan (stress hardening) or on the contrary a lower modulus (stress softening). The PCL/PVA construct was barely affected by fatigue, and showed same mechanical response throughout the fatigue times (Figure $6.4 \mathrm{~d}, 6.5 \mathrm{~d}, 6.6 \mathrm{~d}$ ). The hysteresis curve showed constant plastic strain energy density for the PCL/PVA construct and the PVA hydrogel itself (Figure 6.8). The dry and water immersed PCL scaffold show higher plastic strain energy density that decreased for increasing fatigue cycles, indicating that the porosity is continuously decreasing. The mechanical experiments on the PCL/PVA construct show overall stable mechanical behavior, indicating that once the scaffold is filled with repair tissue, the scaffold/tissue construct can withstand long-term in vivo conditions.

In the study of P(EA-HEA) scaffolds with different crosslinking density, it was seen that the polymer 
crosslinking density highly affected the mechanical behaviour (Figure 4.2 a-c). For scaffolds with different crosslinking density, the mechanical behaviour changed significantly between empty and water immersed scaffolds. The pore size and porosity differed slightly between the samples, but not sufficient to explain the increase in elastic modulus by water filling (Figure 4.1 ac). This means that the water flow through the scaffold is dependent on the proper material toughness, and this effect is less important in a hard material. For the softest material, with less crosslinking density, the water motion through the scaffolds macro pores increased the elastic modulus by 7 times. This can be compared to the stiffest material that increased the modulus 3 times by having water in the pores. When the P(EA-HEA) scaffold was filled with PVA hydrogel and subjected to different number of cycles of freezing and thawing, the modulus decreased for the soft hydrogel (Figure 4.2). This is the same pattern as for the PCL scaffolds (Figure 3.5). An important difference was seen however, and it was that the modulus for the P(EA-HEA)/PVA for 6 cycles of $\mathrm{f} / \mathrm{t}$ did not increase compared to the water-filled scaffolds. On the contrary, the modulus for the PCL/PVA 6 cycles $\mathrm{f} / \mathrm{t}$ increased significantly to the water immersed samples. By having both macro and micro-porosity it seems like the 
densely crosslinked hydrogel filling the pores of PCL impedes water flow through the construct and increases the modulus. This was only seen for the hydrogel filled samples, and a quite opposite phenomenon was observed in the water immersed samples. The dry and water immersed PCL scaffolds show practically the same value of elastic modulus (Figure 3.5), whereas the P(EA-HEA) scaffolds show a great value increase by having water in the pores (Figure 4.2). By having both macro and microporosity, the water expulsion from the PCL scaffold was facilitated and this lowered the modulus to dry sample values. This is very interesting since it shows the importance of micro-porosity in the scaffolds wall. In an implanted scaffold in a chondral defect, this would mean that the scaffold initially facilitates water flow through the pores. Once the repair tissue would grow inside the scaffolds pores, the scaffold/tissue construct approaches its mechanical properties to adjacent healthy tissue.

Regarding the in vivo studies of the PCL implant device with subchondral bone anchoring: The macroscopic study showed that in most of the defects a repair tissue was covering the articular surface completely or almost completely. Besides, only 16\% presented a cystic cavity, which was in the deeper end of the pin. These results 
showed an enhanced tissue repair and diminished subchondral bone alterations from the trial where only PCL scaffold was implanted without anchoring pin. The defects with only scaffold implanted showed high incidence of cystic cavities. The microfracture controls also showed high presence of cyst formation. This shows the importance of subchondral bone anchoring for both tissue repair and foremost, to avoid subchondral bone alterations after microfracture surgery. Alterations such as cyst formations are normally seen when the subchondral bone is perforated and by implanting a biodegradable PLLA pin these are drastically diminished. The immature cartilaginous tissue seen in the defects show that the PCL scaffold is a promising implant device, although modifications need to be done to assure the scaffold fixation. 


\section{Conclusions}

An experimental model for resembling the in vivo behavior of porous scaffolds in a cartilage defect has been developed. The artificial cartilage model can be employed for predicting the mechanical behavior of porous implants. By introducing an aqueous of PVA in the pores of the scaffold and subject the construct to different number of freezing and thawing cycles, interesting features can be seen. First of all, it is possible to tailor the mechanical properties of the porous scaffolds with hydrogel filling, up to values of human articular cartilage. The mechanical behavior of the hydrogel filled scaffolds is different for scaffolds with macro-porosity and scaffolds with both macro and micro-porosity. The micro-porosity plays an important role in the hydrogel filled samples, by increasing the mechanical properties. The gel inside the micro-pores hinders water flow through them. Microporosity is also seen to play an important role in the permeability of the scaffolds, with higher influence than hydrogel filling. Micro-porosity is also important in the scaffolds that are immersed in water. The scaffolds with solely macro-porosity increase the elastic modulus up to seventh fold by having water in the pores. However, for scaffolds that have double pore architecture, the elastic 
modulus is similar for empty or water filled scaffolds. On the other hand, by the experimental model other interesting features are observed. When the scaffold/hydrogel construct is subjected to low number of $\mathrm{f} / \mathrm{t}$ cycles, the elastic modulus decrease. This means that the soft hydrogel facilitates the water expulsion from the scaffold, and lower the modulus to values below the water immersed scaffolds. Not until the hydrogel is readily crosslinked, the modulus increase. The modulus then increase to values, for the double pore architecture scaffold, higher than the water immersed scaffold, but for the solely macro-porous scaffold to values in the same order as the water immersed scaffolds. Once again the micro-porosity was shown to play an important role in the mechanical behavior of the scaffolds. Then, the PCL/PVA scaffold was tested in long-term mechanical behavior experiments, showing the stability of the construct. The experimental model with PVA hydrogel as cartilage model is considered a good artificial model, allowing simulating in vivo conditions. The animal studies of the PCL scaffold show that the biological results are better when implanting with an anchoring device to the subchondral bone. The results both for solely PCL scaffold or scaffold and PLLA pin are better than the controls of only microfracture surgery, in the case of neotissue formation 
and repair tissue formed. Foremost, the cyst formations and subchondral bone alterations highly decrease when implanting the scaffold with an anchoring system. This is important since subchondral bone plate alterations is an unsolved clinical problem in cartilage engineering. Problems with cyst formation diminish when the PLLA dart was anchored to the subchondral bone. This is promising for future pre-clinical trials. The fixation device need to be improved to assure that the scaffold stay in place in the chondral defect. 


\section{Future work}

The PVA artificial cartilage model is well characterized and is applied on both a micro- and macro porous PCL scaffold and a macro porous P(EA-HEA) scaffold. The PVA model could be used in other scaffold systems, to predict the mechanical outcome of the implanted scaffold. In this way, unnecessary animal sacrifice is avoided. It could be interested to study the degradation of the polymers with this cartilage model, to evaluate the implant stability during in vivo degradation.

The PCL scaffold was synthetized according to a patented implant, a device consistent of a chondral part, a porous sponge, attached to a biocompatible anchoring system to the subchondral bone. The results from the in vivo studies of this device show that the product development needs to improve for use in animal studies. By improving the attachment to the PLLA pin better results are believed to be obtained.

In humans however, the thicker cartilage will allow the chondral part to consist of a polymeric membrane attaching the sponge. It could also be of interest to use an anchoring system of even smaller diameter, to try to damage the subchondral bone as little as possible. 


\section{Publications}

\section{Directly related to the thesis}

- An in vitro experimental model to predict the mechanical behavior of macroporous scaffolds implanted in articular cartilage.

Vikingsson L, Gómez-Tejedor JA, Gallego Ferrer G, Gómez Ribelles JL.

J Mech Behav Biomed Mater. 2014;32:125-31

- Relationship between micro porosity, water permeability and mechanical behavior in scaffolds for cartilage engineering.

Vikingsson L, Claessens B, Gómez-Tejedor JA, Gallego Ferrer G, Gómez Ribelles JL.

J Mech Behav Biomed Mater. 2015;48:60-69

- An experimental fatigue study of a porous scaffold for the regeneration of articular cartilage.

Vikingsson L, Gómez-Tejedor JA, Gallego Ferrer G, Gómez Ribelles JL.

J Biomechanics. 2015;48:1310-1317 
- Prediction of the "in vivo" mechanical behaviour of biointegrable acrylic macroporous scaffolds

Vikingsson L, Antolinis Turpin C, Gómez-Tejedor JA, Gallego Ferrer G, Gómez Ribelles JL.

Accepted for publication in Materials Science and Engineering C 2015. June 2015.

\section{Collaborations related to the thesis}

- In vitro mechanical fatigue behavior of poly- $\varepsilon$ caprolactone macroporous scaffolds for cartilage tissue engineering: influence of pore filling by a poly(vinyl alcohol) gel.

Panadero JA, Vikingsson L, Gomez Ribelles JL, Lanceros-Mendez S, Sencadas V.

J Biomed Mater Res B Appl Biomater. 2014.09

- Fatigue prediction in fibrin poly- $\varepsilon$-caprolactone macroporous scaffolds.

Panadero JA, Vikingsson L, Gomez Ribelles JL, Sencadas V, Lanceros-Mendez S.

J Mech Behav Biomed Mater. 2013; 28:55-61 


\section{Not directly related to the thesis}

- Combining self-assembling peptide gels with threedimensional elastomer scaffolds.

Vallés-Lluch A, Arnal-Pastor M, Martínez-Ramos C, Vilariño-Feltrer G, Vikingsson L, Castells-Sala C, Semino CE, Monleón Pradas M.

Acta Biomater. 2013;9(12):9451-60.

- Grid polymeric scaffolds with polypeptide gel filling as patches for infarcted tissue regeneration.

Vallés-Lluch A, Arnal-Pastor M, Martínez-Ramos C, Vilariéño-Feltrer G, Vikingsson L, Monleón Pradas M.

Conf Proc IEEE Eng Med Biol Soc. 2013;2013:69614.

\section{Conferences}

- In vivo response of a biomedical implant device for articular cartilage regeneration GoLife 2015, Gothenburg Dec 2015

L. Vikingsson, M. Sancho-Tello, S. Martínez Díaz , F. García, J.A. Gómez-Tejedor, G. Gallego Ferrer, C. Carda, J.C Monllau ,J.L. Gómez Ribelles 
- Histopathological and morphometric evaluation of articular cartilage regeneration with a biomaterial implant in an ovine model

VI International Conference on Histology and Tissue Engineering

Bilbao, 16-18 Sept 2015

Ruiz-Saurí, A.; Sancho-Tello Valls, M.; Martín

Buigues, D.; Martín de Llano, J.J.; Mata Roig, M.;

Vikingsson, L.; Gómez-Tejedor, J.A.; Gallego Ferrer,

G; Gómez Ribelles, J.L.; Carda Batalla, C.

- Tissue Engineering for cartilage regeneration in large mammals

VI International Conference on Histology and Tissue Engineering

Bilbao, 16-18 Sept 2015

Sancho-Tello Valls, M.; Vikingsson, L.; Martínez

Díaz, S.; García, F.; Gómez-Tejedor, J.A.; Gallego Ferrer, G.; Ruiz Saurí, A.; Monllau, J.C.; Gómez Ribelles, J.L.; Carda Batalla, C. 
- Role of microporosity on the mechanical performance of gel-filled PCL scaffolds for cartilage regeneration European Society for Artificial Organs.XLII Annual ESAO Conference, $2^{\text {nd }}-5^{\text {th }}$ September 2015, Leuven, Belgium

Line Vikingsson, Babeth Claessens, José Antonio Gómez-Tejedor, Gloria Gallego Ferrer, José Luis Gómez Ribelles

- Fatigue studies of a scaffold and hydrogel construct for cartilage engineering $3^{\text {rd }}$ Belgian Symposium on Tissue Engineering BSTE $201519^{\text {th }}$ and 20 $0^{\text {th }}$ of March of 2015, KU Leuven, Belgium

L. Vikingsson, G. Gallego Ferrer ,J.A..GómezTejedor, J.L. Gómez Ribelles

- A scaffold and hydrogel construct with cartilage like mechanical properties for tissue engineer purpose 8th Ciber-BBN Annual Conference, 10-11 November 2014, Girona, Spain

L. Vikingsson, G. Gallego Ferrer, J.A..GómezTejedor, J.L. Gómez Ribelles 
- Diseno de soportes macroporosos para reparación osea producidos con tecnología de impresión $3 D$

I Congreso Biomedicina Predocs Valencia, 27-28 November 2014, Valencia M.Oliver Gasch, L. Vikingsson, J.L. Gómez Ribelles

- A Poly( caprolactone) scaffold filled with poly(vinyl alcohol) gel can mimic the mechanical behaviour of articular cartilage

BSTE 2nd Belgium Symposium on Tissue Engineering 24-25 October 2013 Loeven, Belgium Vikingsson L, Gómez-Tejedor JA, Gallego Ferrer G, Gómez Ribelles JL.

- In vitro experimental model to assess long-term performance of macroporous scaffolds implanted in soft or hard tissues

Frontiers in Polymer Science in association with the journal Polymer, 21-23 May 2013, Sitges, Spain Vikingsson L, Antolinis Turpin C, Gómez-Tejedor JA, Gallego Ferrer G, Gómez Ribelles JL. 
- Combination of self-assembling peptides and scaffolds: characterization, protein delivery and biological performance

World Conference of Regenerative Medicine 2-4 November 2011 Leipzig, Germany.

M Monleon-Pradas, A Valles-Lluch, L Vikingsson, C Martinez-Ramos , M Arnal-Pastor, M Perez-Garnes, D Garcia-Cruz and JL Escobar-Ivirico

- Degradation of polycaprolactone-based networks containing a hydrophilic component 25th European Conference on Biomaterials (Madrid, September 8-12th, 2013)

Juan Manuel Fernández, Line Vikingsson, José Luis Gómez Ribelles , Ana Cortizo

- Estudio de degradacion de material polimerico para regenracion de tejido oseo cartilaginoso Sociedad Argentina de Inmunologia Annual meeting 14-17 November 2012

Fernandez J, Vikingsson L, Cortiz A, Gómez Ribelles, JL 
- Degradation of polycaprolactone-based networks containing a hydrophilic component.

25th European Conference on Biomaterials September 8th - 12th, Madrid 2013

Fernandez JM, Vikingsson L, Gómez Ribelles JL, Cortizo AM

- Estudios de degradación de redes de policaprolactona y ácido poliláctico con poliacrilato de hidroxietilo para ingeniería de tejidos.

VIII Reunión Anual de la Sociedad Argentina de Investigación Clínica 2013

Fernandez JM, Vikingsson L, Oberti TG, Gómez Ribelles JL, Cortiz A 


\section{References}

1. Gray H. Anatomy of the Human Body. 20th Editi. Lewis WH, editor. Philadelphia: Lea \& Febiger; 1918.

2. Fell HB. The histogenesis of cartilage and bone in the long bones of the embryonic fowl. J Morphol Physiol. 1925;40:417-59.

3. Goldring MB, Tsuchimochi K, ljiri K. The control of chondrogenesis. J Cell Biochem. United States; 2006 Jan;97(1):33-44.

4. Holtzer H. Control of Chondrogenesis in the Embryo. Biophys J [Internet]. Elsevier; 2015 Mar 23;4(1):239-50.

5. Joyce ME, Roberts AB, Sporn MB, Bolander ME. Transforming growth factor-beta and the initiation of chondrogenesis and osteogenesis in the rat femur. J Cell Biol; 1990 Jun;110(6):2195-207.

6. Martin I, Jakob M, Schafer D, Dick W, Spagnoli G, Heberer M. Quantitative analysis of gene expression in human articular cartilage from normal and osteoarthritic joints. Osteoarthr Cartil. 2001;9(2):112-8.

7. Maroudas A. Physicochemical properties of articular cartilage. Adult Articular Cartilage. Freeman MA. Kent, UK: Pitman Medical; 1979. p. 215-90.

8. Mankin HJ, Thrasher AZ. Water content and binding in normal and osteoarthritic human cartilage. J Bone Jt Surg. 1975;57:76-80.

9. Nap R, Szleifer I. Structure and interactions of aggrecans: statistical thermodynamic approach. Biophys J. 2008;95(10):4570-84.

10. Fox AJC, Bedi A, Rodeo AA. The Basic Science of Human Knee Menisci Structure, Composition, and Function. Sport Heal A Multidiscip Approach. 2011;4:340-51. 
11. Kiani C, Chen L, Wu YJ, Yee AJ, Yang BB. Structure and function of aggrecan. Cell Res. China; 2002 Mar;12(1):1932.

12. Levett PA, Melchels FPW, Schrobback K, Hutmacher DW, Malda J, Klein TJ. A biomimetic extracellular matrix for cartilage tissue engineering centered on photocurable gelatin, hyaluronic acid and chondroitin sulfate. Acta Biomater [Internet]. 2014 Jan;10(1):214-23.

13. Uebelhart D, Williams JM. Effects of hyaluronic acid on cartilage degradation. Curr Opin Rheumatol.; 1999 Sep;11(5):427-35.

14. Erwin WM, Hood KE. The cellular and molecular biology of the intervertebral disc: A clinician's primer. J Can Chiropr Assoc [Internet]. Canadian Chiropractic Association; 2014 Sep;58(3):246-57.

15. Eyre DR, Wu JJ. Type XI or $1 \alpha 2 \alpha 3 \alpha$ collagen. Academic $P$. Mayne R, Burgeson R, editors. Structure and Function of Collagen Types. New York; 1987. 261-281 p.

16. Mendler M, Eich-Bender SG, Vaughan L, Winterhalter KH Bruckner P. Cartilage contains mixed fibrils of collagen types II, IX, and XI. J Cell Biol. 1989;108:191-7.

17. Klein TJ, Malda J, Sah RL, Hutmacher DW. Tissue Engineering of Articular Cartilage with Biomimetic Zones. Tissue Eng Part B-Rev. 2009;15(2):143-57.

18. Eyre D. Collagen of articular cartilage. Arthritis Res. 2002;4:30-5.

19. Lane JM, Weiss C. Review of articular cartilage collagen research. Arth Rheum. 1975;18:553-62.

20. Linn F, Sokolof FL. Movement and composition of interstitial fluid of cartilage. Arth Rheum. 1965;8:481-94.

21. Buckwalter JA, Mow VC, Ratcliffe A. Restoration of Injured or Degenerated Articular Cartilage. J Am Acad Orthop Surg, . 1994;2(4):192-201. 
22. Hunziker EB, Quinn TM, Hauselmann HJ. Quantitative structural organization of normal adult human articular cartilage. Osteoarthr Cartil. 2002;10(7):564-72.

23. Yang PJ, Temenoff JS. Engineering Orthopedic Tissue Interfaces. Tissue Eng Part B-Rev. 2009;15(2):127-41.

24. Nguyen LH, Kudva AK, Guckert NL, Linse KD, Roy K. Unique biomaterial compositions direct bone marrow stem cells into specific chondrocytic phenotypes corresponding to the various zones of articular cartilage. Biomaterials. 2011;32(5):1327-38.

25. Jurvelin JS, Buschmann MD, Hunziker EB. Mechanical anisotropy of the human knee articular cartilage in compression. Proc Inst Mech Eng H. 2003;217(3):215-9.

26. Akizuki S, Mow VC, Muller F, Pita JC, Howell DS, Manicourt $\mathrm{DH}$. Tensile properties of human knee joint cartilage: I. Influence of ionic conditions, weight bearing, and fibrillation on the tensile modulus. J Orthop Res. 1986;4:379-92.

27. Schinagl RM, Gurskis D, Chen AC, Sah RL. Depthdependent confined compression modulus of fullthickness bovine articular cartilage. J Orthop Res. 1997;15:499-506.

28. Elder BD, Athanasiou KA. Hydrostatic pressure in articular cartilage tissue engineering: from chondrocytes to tissue regeneration. Tissue Eng Part B Rev. 2009;15(1):43-53.

29. Wong M, Carter DR. Articular cartilage functional histomorphology and mechanobiology: a research perspective. Bone. 2003;33(1):1-13.

30. Eckstein F, Lemberger B, Gratzke C, Hudelmaier M, Glaser $\mathrm{C}$, Englmeir $\mathrm{KH}$, et al. In vivo cartilage deformation after different types of activity and its dependence on physical training status. Ann Rheum Dis. 2005;64:291-5.

31. Kempson GE, Muir H, Swanson SA V, Freeman MAR. Correlations between stiffness and the chemical constituents of cartilage on the human femoral head. Biochim Biophys Acta - Gen Subj. 1970;2015(1):70-7. 
32. Lu XL, Mow VC. Biomechanics of Articular Cartilage and Determination of Material Properties. Am Coll Sport Med. 2008;40(2):193-9.

33. Poole A, Rosenberg L, Reiner A, lonescu M, Bogoch E, Roughley P. Contents and distributions of the proteoglycans decorin and biglycan in normal and osteoarthritic human articular cartilage. J Orthop Res. 1996;14:681-9.

34. Shirazi R, Shirazi-Adl A. Deep Vertical Collagen Fibrils Play a Significant Role in Mechanics of Articular Cartilage. J Orthop Res. 2008;26(5):608-15.

35. Kempson GE, Freeman MAR, Swanson SA V. The tensile properties of articular cartilage. Nature. 1968;220:1127-8.

36. Jurvelin JS, Buschmann MD, Hunziker EB. Optical and mechanical determination of Poisson's ratio of adult bovine humeral articular cartilage. J Biomech. 1997;30:235-41.

37. Quinn TM, Grodzinsky AJ, Buschmann MD, Kim YJ, Hunziker EB. Mechanical compression alters proteoglycan deposition and matrix deformation around individual cells in cartilage explants. J Cell Sci. ENGLAND; 1998 Mar;111 (5):573-83.

38. Koob TJ, Clark PE, Hernandez DJ, Thurmond FA, Vogel KG. Compression loading in vitro regulates proteoglycan synthesis by tendon fibrocartilage. Arch Biochem Biophys. UNITED STATES; 1992 Oct;298(1):303-12.

39. Gillard GC, Reilly HC, Bell-Booth PG, Flint MH. The influence of mechanical forces on the glycosaminoglycan content of the rabbit flexor digitorum profundus tendon. Connect Tissue Res.; 1979;7(1):37-46.

40. Mow VC, Kuei SC, Lai WM, Armstrong CG. Biphasic Creep and Stress Relaxation of Articular Cartilage in Compression: Theory and Experiments. J Biomech Eng [Internet]. 1980 Feb 1;102(1):73-84.

41. Mow VC, Mansour JM. The permeability of articular cartilage under compressive strain and at high pressures, . J Bone Jt Surg. 1976;58-A(4):509-16. 
42. Mow VC, Holmes MH, Lai M. Fluid transport and mechanical properties of articular cartilage. J Biomech. 1984;17(5):37794.

43. Mak A. The apparent viscoelastic behavior of articular cartilage--the contributions from the intrinsic matrix viscoelasticity and interstitial fluid flows. J Biomech Eng. 1986;108(2):123-30.

44. Mow VC, Lai WM. Recent Developments in Synovial Joint Biomechanic. SI AM Rev. 1980;22:275.

45. Mow VC, Roth V, Armstrong CG. Biomechanics of Joint Cartilage. In: Frankel VH, Nordin MA, editors. Basic Biomechanics of the Skeletal System. Philadelphia: Lea and Febiger; 1980.

46. Lai WM, Hou JS, Mow VC. A triphasic theory for the swelling and deformation behaviors of articular cartilage. J Biomech Eng. UNITED STATES; 1991 Aug;113(3):245-58.

47. Donnan FG. The theory of membrane equilibria. Chem Rev. 1924;1:73-90.

48. Mow VC, Ateshian GA, Lai WM, Sun DN, Wang CB, Gu WY. Effects of fixed charge density on the stress-relaxation behavior of hydrated soft tissues in confined compression. Int J Solids Struct. 1998;35:4945-62.

49. Lu XL, Miller C, Chen FH, Guo XE, Mow VC. The generalized triphasic correspondence principle for simultaneous determination of the mechanical properties and proteoglycan content of articular cartilage by indentation. J Biomech. 2006;40:2434-41.

50. Lai WM, Mow VC, Roth V. Effects of Nonlinear StrainDependent Permeability and Rate of Compression on the Stress Behavior of Articular Cartilage. J Biomech Eng. 1981;103:61.

51. Maroudas A. Permeability of articular cartilage. Nature. 1968;219:1260-1. 
52. Gannon AR, Nagel T, Kelly DJ. The role of the superficial region in determining the dynamic properties of articular cartilage. Osteoarthr Cartil [Internet]. 2012 Nov;20(11):1417-25.

53. Collins DH. The Pathology of Articular and Spinal Diseases. London: Edward Arnold \& Co.; 1949.

54. Browner TD, Akahoahi Y, Orlic P. The Diffusion of Dyes Through Articular Cartilage in Vivo. J Bone Jt Surgery. 1962;44A:456.

55. Ekholm R. Articular Cartilage Nutrition. Acta Anat (Basel). 1951;Supplement.

56. Ekholm R. Nutrition of Articular Cartilage- An Radioautographic Study. Acta Anat (Basel). 1955;24:329.

57. McKibbin, Holdsworth FW. The Nutrition of Immature Joint Cartilage in the Lamb. J Bone Jt Surgery. 1966;48B:793.

58. Lories RJ, Luyten FP. The bone-cartilage unit in osteoarthritis. Nat Rev Rheumatol. 2011;7:43-9.

59. Arkill KP, Winlove CP. Solute transport in the deep and calcified zones of articular cartilage. Osteoarthr Cart. 2008;16(6):708-14.

60. McCutchen CW. The frictional properties of animal joints. Wear. 1962;5(2):1-17.

61. Maroudas A. Physicochemical Properties of Cartilage in the Light of lon Exchange Theory. Biophys J. 1968;8(5):575-95.

62. Soltz MA, Ateshian GA. Experimental verification and theoretical prediction of cartilage interstitial fluid pressurization at an impermeable contact interface in confined compression. J Biomech. 1998;31:927-34.

63. Athanasiou KA, Rosenwasser MP, Buckwalter JA, Malinin TI, Mow VC. Interspecies comparisons of in situ intrinsic mechanical properties of distal femoral cartilage. J Orthop Res. 1991;9(3):330-40. 
64. Korhonen R, Saarakkala S. Biomechanics and Modeling of Skeletal Soft Tissues. Theoretical Biomechanics.

65. Gibson L, Ashby M. Cellular solids: structure and properties. Second. Cambridge University Press; 1997. 175 p.

66. Bowden PB. The elastic modulus of an amorphous glassy polymer. Polymer (Guildf) [Internet]. 1968;9(0):449-54. Available from: http://www.sciencedirect.com/science/article/pii/0032386168 900542

67. Hayes WC, Keer LM, Herrmann G, Mockros LF. A mathematical analysis for indentation tests of articular cartilage. J Biomech. Elsevier; 1972;5(5):541-51.

68. Setton LA, Zhu W, Mow VC. The biphasic poroviscoelastic behavior of articular cartilage: role of the surface zone in governing the compressive behavior. J Biomech. 1993;26(45):581-92.

69. Mak AF, Lai WM, Mow VC. Biphasic indentation of articular cartilage--I. Theoretical analysis. J Biomech.; 1987;20(7):703-14.

70. Flanigan DC, Harris JD, Trinh TQ, Siston RA, Brophy RH. Prevalence of chondral defects in athletes' knees: a systematic review. Med Sci Sports Exerc. United States; 2010 Oct;42(10):1795-801.

71. Mankin HJ, Brandt KD. Biochemistry and metabolism of cartilage in osteoarthritis. In: Moskowitz, AI R et, editors. Osteoarthritis: Diagnosis and Medical/Surgical Management. Philadelphia: WB Co, Saunders; 1992. p. 109-54.

72. Sokolo L. Biology of degenerative joint disease. Chicago: University of Chicago Press; 1969.

73. Maroudas A, Mizrahi J, Katz EP, Wachtel EJ, Soudry M. Physicochemical properties and functional behavior of normal and osteoarthritic human cartilage. In: K. Kuettner et al, editor. Articular Cartilage Biochemistry. New York: Raven Press; 1986. 
74. Eberhardt AW, Lewis JL, Keer LM. Contact of layered elastic spheres as a model of joint contact: effect of tangential load and friction. J Biomech Eng. 1991;113:107-8.

75. Demarteau O, Wendt D, Braccini A, Jakob M, Schafer D, Heberer M, et al. Dynamic compression of cartilage constructs engineered from expanded human articular chondrocytes. Biochem Biophys Res Commun. 2003;310:580-8.

76. Armstrong CG, Mow VC. Variations in the intrinsic mechanical properties of human articular cartilage with age, degeneration, and water content. J Bone Jt Surg. 1982;64A:88-94.

77. Poole A, Kobayashi M, Yasuda T, Laverty S, Mwale F, Kojima T, et al. Type II collagen degradation and its regulation in articular cartilage in osteoarthritis. Ann Rheum Dis. 2002;61:78-81.

78. Sharma L, Kapoor D. Epidemiology of osteoarthritis. In: Moskowitz RW, Altman RD, Hochberg MC, Buckwalter JA, Goldberg VM, editors. Osteoarthritis, Diagnosis and Medical/ Surgical Management. 4th Editio. Philadelphia; 2007. p. 326.

79. Arthritis of the knee. American Academy of Orthopaedic Surgeons. 2014.

80. Yoshihara Y, Nakamura H, Obata K, Yamada H, Hayakawa $\mathrm{T}$, Fujikawa $\mathrm{K}$, et al. Matrix metalloproteinases and tissue inhibitors of metalloproteinases in synovial fluids from patients with rheumatoid arthritis or osteoarthritis. Ann Rheum Dis. 2000;59:455-61.

81. Little CB, Meeker CT, Golub SB, Lawlor KE, Farmer PJ, Smith SM, et al. Blocking aggrecanase cleavage in the aggrecan interglobular domain abrogates cartilage erosion and promotes cartilage repair. J Clin Invest. 2007;117:162736.

82. Kawaguchi $\mathrm{H}$. Endochondral ossification signals in cartilage degradation during osteoarthritis progression in 
experimental mouse models. Mol Cells. Korea (South); 2008 Feb;25(1):1-6.

83. Abramson S, Attur M. Developments in the scientific understanding of osteoarthritis. Arthritis Res Ther [Internet]. 2009;11(3):227.

84. Bollet AJ, Nance JL. Biochemical findings in normal and osteoarthritic articular cartilage. II. Chondroitin sulfate concentration and chain length, water and ash content. J Clin Invest. 1966;45:1170-7.

85. Brittberg M. Which lesions should be treated and why? In: Brittberg M, Gobbi A, Imhoff A, Kon E, Madry H, editors. Cartilage repair: Clinical guidelines-Decision making in cartilage repair-Variable influencing the choice of treatment. First edit. DJO Publications; 2012. p. 3-14.

86. Hjelle K, Solheim E, Strand T, Muri R, Brittberg M. Articular cartilage defects in 1,000 knee arthroscopies. Arthroscopy. United States; 2002 Sep;18(7):730-4.

87. Gobbi A. Age based managment of small $\left(<2 \mathrm{~cm}^{\wedge} 2\right)$ cartilage lesions. In: Brittberg M, Gobbi A, Imhoff AB, Kon E, Madry $\mathrm{H}$, editors. Cartilage repair: Clinical guidelines-Decision making in cartilage repair-Variable influencing the choice of treatment. First edit. DJO Publications; 2012. p. 15-29.

88. Kon E, Filardo G, Berruto M, Benazzo F, Zanon G, Della Villa $S$, et al. Articular cartilage treatment in high-level male soccer players: a prospective comparative study of arthroscopic second-generation autologous chondrocyte implantation versus microfracture. Am J Sports Med. United States; 2011 Dec;39(12):2549-57.

89. A Patient's Guide to Articular Cartilage Problems of the Knee. Houston Methodist Orthopedics \& Sports Medicine. 2003.

90. Tew S, Redman S, Kwan A, Walker E, Khan I, Dowthwaite $G$, et al. Differences in repair responses between immature and mature cartilage. Clin Orthop Relat Res. United States; 2001 Oct;(391 Suppl):S142-52. 
91. Kreuz PC, Erggelet C, Steinwachs MR, Krause SJ, Lahm A, Niemeyer $P$, et al. Is microfracture of chondral defects in the knee associated with different results in patients aged 40 years or younger? Arthroscopy. United States; 2006 Nov;22(11):1180-6.

92. Pridie $\mathrm{KH}$. A method of resurfacing osteoarthritic knee joints. J Bone Jt Surg Br. 1959;41:618-9.

93. Insall J. The Pridie debridement operation for osteoarthritis of the knee. Clin Orthop Relat Res. 1974;(101):61-7.

94. Steadman JR, Rodkey WG, Briggs KK, Rodrigo JJ. The microfracture technique to treat full thickness articular cartilage defects of the knee. Orthopade. Springer; 1999;28(1):26-32.

95. Steadman JR, Rodkey WG, Rodrigo JJ. "Microfracture": surgical technique and rehabilitation to treat chondral defects. Clin Orthop Relat Res. 2001;391:362-9.

96. Steadman JR, Briggs KK, Rodrigo JJ, Kocher MS, Gill TJ, Rodkey WG. Outcomes of microfracture for traumatic chondral defects of the knee: average 11-year follow-up. Arthroscopy. United States; 2003;19(5):477-84.

97. Minas T, Gomoll AH, Rosenberger R, Royce RO, Bryant T. Increased failure rate of autologous chondrocyte implantation after previous treatment with marrow stimulation techniques. Am J Sports Med. United States; 2009 May;37(5):902-8.

98. Frisbie DD, Trotter GW, Powers BE, Rodkey WG, Steadman JR, Howard RD. Arthroscopic subchondral bone plate microfracture technique augments healing of large osteochondral defects in the radial carpal bone and medial femoral condyle of horses. Vet Surg. 1999;28:242-55.

99. Shapiro F, Koide S, Glimcher MJ. Cell origin and differentiation in the repair of full-thickness defects of articular cartilage. J Bone Joint Surg Am.; 1993 Apr;75(4):532-53. 
100. Steinwachs M, Waibl B. Autologous matrix associated chondroneogenesis and matrix associated chondrocyte implantation. In: Brittberg M, Gobbi A, Imhoff AB, Kon E, Madry $\mathrm{H}$, editors. Cartilage repair: Clinical guidelinesDecision making in cartilage repair-Variable influencing the choice of treatment. First edit. DJO Publications; 2012. p. 57-70.

101. Pittenger MF, Mackay AM, Beck SC, Jaiswal RK, Douglas $\mathrm{R}$, Mosca JD, et al. Multilineage potential of adult human mesenchymal stem cells. Science. UNITED STATES; 1999 Apr;284(5411):143-7.

102. Wexler SA, Donaldson C, Denning-Kendall P, Rice C, Bradley $B$, Hows JM. Adult bone marrow is a rich source of human mesenchymal "stem" cells but umbilical cord and mobilized adult blood are not. Br J Haematol. England; 2003 Apr;121(2):368-74.

103. Haynesworth SE, Goshima J, Goldberg VM, Caplan AI. Characterization of cells with osteogenic potential from human marrow. Bone.; 1992;13(1):81-8.

104. Caplan Al. Mesenchymal stem cells. J Orthop Res. 1991 Sep;9(5):641-50.

105. Kuznetsov SA, Krebsbach PH, Satomura K, Kerr J, Riminucci M, Benayahu D, et al. Single-colony derived strains of human marrow stromal fibroblasts form bone after transplantation in vivo. J Bone Miner Res. 1997 Sep;12(9):1335-47.

106. Davies BM, Morrey ME, Mouthuy P-A, Baboldashti NZ, Hakimi O, Snelling $S$, et al. Repairing damaged tendon and muscle: are mesenchymal stem cells and scaffolds the answer? Regen Med. England; 2013 Sep;8(5):613-30.

107. Hass R, Kasper C, Böhm S, Jacobs R. Different populations and sources of human mesenchymal stem cells (MSC): A comparison of adult and neonatal tissue-derived MSC. Cell Commun Signal [Internet]. BioMed Central; 2011 May 14;9:12. 
108. Brittberg M, Lindahl A, Nilsson A, Ohlsson C, Isaksson O, Peterson L. Treatment of deep cartilage defects in the knee with autologous chondrocyte transplantation. N Engl J Med. 1994 Oct;331(14):889-95.

109. Pestka J, Schmal H, Salzmann G, Hecky J, Südkamp N, Niemeyer $P$. In vitro cell quality of articular chondrocytes assigned for autologous implantation in dependence of specific patient characteristics. Arch Orthop Trauma Surg [Internet]. Springer-Verlag; 2011;131(6):779-89.

110. Brittberg $M$. Autologous chondrocyte implantation. In: Brittberg M, Gobbi A, Imhoff AB, Kon E, Madry H, editors. Cartilage repair: Clinical guidelines-Decision making in cartilage repair-Variable influencing the choice of treatment. First edit. DJO Publications; 2012. p. 49-56.

111. Langer R, Vacanti JP. Tissue engineering. Science (80- ). 1993;260:920-6.

112. Hutmacher DW. Scaffolds in tissue engineering bone and cartilage. Biomaterials. Elsevier; 2000;21(24):2529-43.

113. Chiquet M, Renedo AS, Huber F, Flück M. How do fibroblasts translate mechanical signals into changes in extracellular matrix production? Matrix Biol. Germany; 2003;22(1):73-80.

114. Bryant S, Chowdhury T, Lee D, Bader D, Anseth K. Crosslinking density influences chondrocyte metabolism in dynamically loaded photocrosslinked poly(ethylene glycol) hydrogels. Ann Biomed Eng . 2004;32(407-417).

115. Wang Y, de Isla N, Huselstein C, Wang B, Netter P, Stoltz J, et al. Effect of alginate culture and mechanical stimulation on cartilaginous matrix synthesis of rat dedifferentiated chondrocytes. Biomed Mater Eng. 2008;18:47-54.

116. Appelman T, Mizrahi J, Elisseeff J, Seliktar D. The influence of biological motifs and dynamic mechanical stimulation in hydrogel scaffold systems on the phenotype of chondrocytes. Biomaterials. 2011;32:1508-16. 
117. Lebourg M, Suay Antón J, Gómez Ribelles JL. Porous membranes of PLLA-PCL blend for tissue engineering applications. Eur Polym J [Internet]. 2008 Jul;44(7):2207-18.

118. Hollister SJ. Porous scaffold design for tissue engineering. Nat Mater. 2005;4(7):518-24.

119. Hutmacher DW. Scaffold design and fabrication technologies for engineering tissues - state of the art and future perspectives. J Biomater Sci-Polym Ed. 2001;12(1):107-24.

120. Buschmann MD, Kim YJ, Wong M, Frank E, Hunziker EB, Grodzinsky AJ. Stimulation of aggrecan synthesis in cartilage explants by cyclic loading is localized to regions of high interstitial fluid flow. Arch Biochem Biophys.; 1999 Jun;366(1):1-7.

121. Santamaría VA, Deplaine $H$, Mariggió $D$, Villanueva-Molines AR, García-Aznar JM, Gómez Ribelles JL, et al. Influence of the macro and micro-porous structure on the mechanical behavior of poly (I-lactic acid) scaffolds. J Non Cryst Solids. Elsevier; 2012;358(23):3141-9.

122. Deplaine H, Lebourg M, Ripalda P, Vidaurre A, Sanz-Ramos $\mathrm{P}$, Mora $\mathrm{G}$, et al. Biomimetic hydroxyapatite coating on pore walls improves osteointegration of poly(L-lactic acid) scaffolds. J Biomed Mater Res B Appl Biomater. United States; 2013;101(1):173-86.

123. Lebourg M, Suay Antón J, Gómez Ribelles JL. Hybrid structure in PCL-HAp scaffold resulting from biomimetic apatite growth. J Mater Sci Mater Med.; 2010;21(1):33-44.

124. Mantila Roosa SM, Kemppainen JM, Moffitt EN, Krebsbach $\mathrm{PH}$, Hollister SJ. The pore size of polycaprolactone scaffolds has limited influence on bone regeneration in an in vivo model. J Biomed Mater Res Part A. 2010;92A(1):359-68.

125. Sancho-Tello M, Forriol F, Gastaldi P, Ruiz-Saurí A, Martín de Llano J, Novella-Maestre E, et al. Time evolution of "in vivo" articular cartilage repair induced by bone marrow stimulation and scaffold implantation in rabbits. Int $\mathrm{J}$ Artif Organs. 2015; 
126. Smith BD, Grande DA. The current state of scaffolds for musculoskeletal regenerative applications. Nat Rev

Rheumatol. 2015;11:213-22.

127. Strauss EJ, Barker JU, Kerscher JS, Cole BJ, Mithoefer K. Augmentation Strategies following the Microfracture Technique for Repair of Focal Chondral Defects. Cartilage. 2010;1(2):145-52.

128. Marcacci M, Berruto M, Brocchetta D, Delcogliano A, Ghinelli D, Gobbi A, et al. Articular cartilage engineering with Hyalograft C: 3-year clinical results. Clin Orthop Relat Res. United States; 2005 Jun;(435):96-105.

129. Niemeyer P, Lenz P, Kreuz PC, Salzmann GM, Sudkamp $\mathrm{NP}$, Schmal $\mathrm{H}$, et al. Chondrocyte-seeded type I/III collagen membrane for autologous chondrocyte transplantation: prospective 2-year results in patients with cartilage defects of the knee joint. Arthroscopy. United States; 2010 Aug;26(8):1074-82.

130. Trial Comparing BST-CarGel and Microfracture in Repair of Articular Cartilage Lesions in the Knee. NCT00314236; 2006.

131. Chevrier A, Hoemann CD, Sun J, Buschmann MD. Chitosan-glycerol phosphate/blood implants increase cell recruitment, transient vascularization and subchondral bone remodeling in drilled cartilage defects. Osteoarthritis Cartilage. England; 2007 Mar;15(3):316-27.

132. Cheng N-C, Estes BT, Awad HA, Guilak F. Chondrogenic differentiation of adipose-derived adult stem cells by a porous scaffold derived from native articular cartilage extracellular matrix. Tissue Eng Part A. United States; 2009 Feb;15(2):231-41.

133. Berdichevski A, Shachaf $Y$, Wechsler R, Seliktar D. Protein composition alters in vivo resorption of PEG-based hydrogels as monitored by contrast-enhanced MRI. Biomaterials [Internet]. $2015 \mathrm{Feb} ; 42(0): 1-10$.

134. Bartha L, Hamann D, Pieper J, Peters F, Riesle J, Vajda A, et al. A clinical feasibility study to evaluate the safety and 
efficacy of PEOT/PBT implants for human donor site filling during mosaicplasty. Eur J Orthop Surg Traumatol. France; 2013 Jan;23(1):81-91.

135. Marcacci M, Zaffagnini S, Kon E, Delcogliano M, Di Martino A, Filardo G, et al. Maioregen. Arch di Ortop e Reumatol [Internet]. Springer Milan; 2009;120(3-4):35-7.

136. Kon E, Filardo G, Perdisa F, Venieri G, Marcacci M. Clinical results of multilayered biomaterials for osteochondral regeneration. J Exp Orthop [Internet]. 2014;1(1):10.

137. Eduardo P, Batista J, Millan-billi A, Patthauer L, Vera S, Gomez-masdeu M, et al. Magnetic resonance evaluation of TruFit $\circledast$ plugs for the treatment of osteochondral lesions of the knee shows the poor characteristics of the repair tissue. Knee [Internet]. Elsevier B.V.; 2014;21(4):827-32.

138. Tomić SL, Mićić MM, Dobić SN, Filipović JM, Suljovrujićb EH. Smart poly(2-hydroxyethyl methacrylate itaconic acid) hydrogels for biomedical application. Radiat Phys Chem. 2010 May;79(5):643-9.

139. Huglin MR. Hydrogels in medicine and pharmacy Edited by N. A. Peppas, CRC Press Inc., Boca Raton, Florida, 1986 (Vol. I),

140. Spiller KL, Laurencin SJ, Charlton D, Maher SA, Lowman AM. Superporous hydrogels for cartilage repair: Evaluation of the morphological and mechanical properties. Acta Biomater. 2008;4:17-25.

141. Hunziker EB. Articular cartilage repair: basic science and clinical progress. A review of the current status and prospects. Osteoarthr Cartil. England; 2002;10(6):432-63.

142. Alio del Barrio JL, Chiesa M, Gallego Ferrer G, Garagorri N, Briz N, Fernandez-Delgado J, et al. Biointegration of corneal macroporous membranes based on poly(ethyl acrylate) copolymers in an experimental animal model. J Biomed Mater Res A. United States; 2015 Mar;103(3):1106-18. 
143. Brittberg M. Cartilage repair: Clinical guidelines-Decision making in cartilage repair-Variable influencing the choice of treatment. First edit. Brittberg M, Gobbi A, Imhoff A, Kon E, Madry H, editors. DJO Publications; 2012.

144. Quarch VMA, Enderle E, Lotz J, Frosch K. Fate of large donor site defects in osteochondral transfer procedures in the knee joint with and without TruFit Plugs. 2014;657-66.

145. Orth P, Cucchiarini M, Kohn D, Madry H. Alterations of the subchondral bone in osteochondral repair--translational data and clinical evidence. Eur Cell Mater. 2013;25:299-316;

146. Gomoll AH, Madry H, Knutsen G, van Dijk N, Seil R, Brittberg $\mathrm{M}$, et al. The subchondral bone in articular cartilage repair: current problems in the surgical management. Knee Surgery, Sport Traumatol Arthrosc [Internet]. Berlin/Heidelberg: Springer-Verlag; 2010 Apr 4;18(4):43447.

147. Kreuz PC, Steinwachs MR, Erggelet C, Krause SJ, Konrad $\mathrm{G}$, Uhl M, et al. Results after microfracture of full-thickness chondral defects in different compartments in the knee. Osteoarthr Cartil [Internet]. 2006 Nov;14(11):1119-25.

148. Peterson L, Minas T, Brittberg M, Lindahl A. Treatment of osteochondritis dissecans of the knee with autologous chondrocyte transplantation: results at two to ten years. J Bone Joint Surg Am. United States; 2003;85-A Suppl:17-24.

149. Brandt KD, Radin EL, Dieppe PA, van de Putte L. Yet more evidence that osteoarthritis is not a cartilage disease. Annals of the rheumatic diseases. England; 2006. p. 1261-4.

150. Burr DB, Radin EL. Microfractures and microcracks in subchondral bone: are they relevant to osteoarthrosis? Rheum Dis Clin North Am. United States; 2003 Nov;29(4):675-85.

151. Rodenas-Rochina J, Gómez Ribelles JL, Lebourg M. Comparative study of PCL-HAp and PCL-bioglass composite scaffolds for bone tissue engineering. J Mater Sci Mater Med. 2013;24:1293-308. 
152. Martinez-Diaz S, Garcia-Giralt N, Lebourg M, GómezTejedor J-A, Vila G, Caceres E, et al. In vivo evaluation of 3dimensional polycaprolactone scaffolds for cartilage repair in rabbits. Am J Sport Med. United States; 2010;38(3):509-19.

153. Lebourg M, Suay Antón J, Gómez Ribelles JL. Porous membranes of PLLA-PCL blend for tissue engineering applications. Eur Polym J. 2008;44(7):2207-18.

154. Lebourg M, Martínez-Díaz S, García-Giralt N, TorresClaramunt R, Gómez Ribelles JL, Vila-Canet G, et al. Cellfree cartilage engineering approach using hyaluronic acidpolycaprolactone scaffolds: A study in vivo. J Biomater Appl [Internet]. 2014;28(9):1304-15.

155. Lebourg M, Rochina JR, Sousa T, Mano J, Gómez Ribelles JL. Different hyaluronic acid morphology modulates primary articular chondrocyte behavior in hyaluronic acid-coated polycaprolactone scaffolds. J Biomed Mater Res A. United States; 2013;101(2):518-27.

156. Garcia-Giralt N, Izquierdo R, Nogues X, Perez-Olmedilla M, Benito P, Gomez-Ribelles JL, et al. A porous PCL scaffold promotes the human chondrocytes redifferentiation and hyaline-specific extracellular matrix protein synthesis. $J$ Biomed Mater Res A. United States; 2008 Jun;85(4):10829.

157. Gómez Ribelles JL, Carda C, Monllau JC. Dispositivo pra fijar un material macroporoso para la regeneración del cartílago articular. EP201131625 PCT/WO2013/178852, 2011. p. 1-30.

158. Hassan CM, Peppas NA. Structure and applications of Poly(vinyl alcohol) Hydrogels produced by conventional crosslinking or by freezing/thawing methods. Adv Polym Sci. Springer; 2000;153:37-65.

159. Tubbs RK. Sequence distribution of partially hydrolyzed poly(vinyl acetate). J Polym Sci A1. 1966;4(3):623-9.

160. Danno A. Gel Formation of Aqueous Solution of Polyvinyl Alcohol Irradiated by Gamma Rays from Cobalt-60. J Phys 
Soc Japan [Internet]. The Physical Society of Japan; 1958 Jul 15;13(7):722-7.

161. Hassan CM, Peppas NA. Cellular PVA Hydrogels Produced by Freeze/Thawing. J Appl Polym Sci. 2000;76:2075-9.

162. Li F, Su YL, Shi DF, Wang CT. Comparison of human articular cartilage and polyvinyl alcohol hydrogel as artificial cartilage in microstructure analysis and unconfined compression. Adv Mater Res. Trans Tech Publ; 2010;87:188-93.

163. Tamura K, Ike O, Hitomi S, Isobe J, Shimizu Y, Nambu M. A New Hydrogel and Its Medical Application. Trans Amer Soc Artif Organs. 1986;32:605.

164. Grant C, Twigg P, Egan A, Moody A, Eagland D, Crowther $\mathrm{N}$, et al. Poly(vinyl alcohol) Hydrogel as a Biocompatible Viscoelastic Mimetic for Articular Cartilage. Biotechnol Prog. 2006;22:1400-6.

165. Paradossi G, Cavalieri F, Chiessi E, Spagnoli C, Cowman MK. Poly(Vinyl Alcohol) as versatile biomaterial for potential biomedical applications. J Mater Sci Mater Med. 2003;14:687-91.

166. Peppas NA. Turbidimetric studies of aqueous poly(vinyl alcohol) solutions. Die Makromol Chemie. Hüthig \& Wepf Verlag; 1975 Nov 1;176(11):3433-40.

167. Hickey AS, Peppas NA. Mesh size and diffusive characteristics of semicrystalline poly(vinyl alcohol) membranes prepared by freezing/thawing techniques. J Membr Sci. 1995;107:229-37.

168. Yokoyama E, Masada I, Shimamura K, Ikawa T, Monobe K. Morphology and structure of highly elastic poly(vinyl alcohol) hydrogel prepared by repeated freezing-and-melting. Colloid Polym Sci. 1986;264:595-601.

169. Eldridge JE, Ferry JD. Studies of the cross-linking process in gelatin gels. III. Dependence of melting point on concentration and molecular weight. J Phys Chem. 1954;52:992. 
170. Komatsu M, Inoue T, Miyasaka K. Dynamical aspects of solgel transition in an aqueous solution of poly(vinyl alcohol). SEN-I GAKKAISHI. 1984;40:316-8.

171. Hatakeyemaa T, Unoa J, Yamadaa C, Kishi A, Hatakeyama $\mathrm{H}$. Gel-sol transition of poly(vinyl alcohol) hydrogels formed by freezing and thawing. Thermochim Acta. 2005;431:1448.

172. Hasegawa R, Takahashi Y, Chatani Y, Tadokoro H. Crystal Structures of Three Crystalline Forms of Poly(vinylidene fluoride). Polym J. 1972;3:600-10.

173. Peppas NA, Stauffer SR. Reinforced uncrosslinked poly (vinyl alcohol) gels produced by cyclic freezing-thawing processes: a short review. J Control Release. 1991;16:30510.

174. Kawanishi K, Komatsu M, Inoue T. Thermodynamic consideration of the sol-gel transition in polymer solutions. Polymer (Guildf) [Internet]. 1987 May;28(6):980-4.

175. Londono ME, Jaramillo JM, Sabater R, Velez JM. Dielectric properties of Poly(Vinyl Alcohol) hydrogels prepared by freezing/thawing technique. EIA. 2012;18:105-14.

176. Watase M, Nishinari K. Rheological and DSC changes in poly( vinyl alcohol) gels induced by immersion in water. J Polym SciPolym Phys. 1985;23:1803-11.

177. Jones Jl. Polyvinyl alcohol. Properties and applications. Edited by C. A. Finch. John Wiley, Chichester. 1973. Pp. xviii + 622. Price: £14.00. Br Polym J [Internet]. John Wiley \& Sons, Ltd; 1973 Nov 1;5(6):493-4.

178. Fukumori T, Nakaoki T. Significant Improvement of Mechanical Properties for Polyvinyl Alcohol Film Prepared from Freeze/Thaw Cycled Gel. Open J Org Polym Mater. 2013;3(110-116).

179. Lozinsky VI, Domotenko L V, Valnerman ES, Rogozhin AM, Mamtsis S V. On the possibility of mechanodestruction of 
poly (vinyl alcohol) molecules under moderate freezing of its concentrated water solutions. Polym Bull. 1986;15(330-340).

180. Keller A. A note on single crystals in polymers: Evidence for a folded chain configuration. Philos Mag. 1957;2(21):11715.

181. Brunn CW. Advances in Colloid Science. Intersci Publ. 1956;2(121).

182. Mooney RCL. An X-Ray Study of the Structure of Polyvinyl Alcohol. J Am Chem Soc. 1941;63(10):2828-32.

183. Brunn CW. Crystal structure of Poly(Vinyl Alcohol). Nature. 1948;161:929-30.

184. Hyon S-H, Cha W-I, Ikada Y. Preparation of transparent poly(vinyl alcohol) hydrogel. Polym Bull [Internet]. SpringerVerlag; 1989;22(2):119-22.

185. Tong H, Noda I, Gryte C. CPS 768 Formation of anisotropic ice-agar composites by directional freezing. Colloid Polym Sci [Internet]. Steinkopff-Verlag; 1984;262(7):589-95.

186. Woessner DE, Snowden BSJ. Pulsed NMR study of temperature hysteresis in agar-water system. J Colloid Interface Sci. 1970;34:290.

187. Hatakeyama T, Yamauchi A. Studies on bound water in Poly(Vinyl Alcohol). Eur Polym J. 1984;20:61-4.

188. Hatakeyama T, Yamauchi A, Hatakeyama H. Effect of thermal hysteresis on structural change of water restrained in poly(vinyl alcohol) pseudo-gel. Eur Polym J [Internet]. 1987;23(5):361-5.

189. Hassan CM, Trakampan P, Peppas NA. Water solubility characteristics of poly(vinyl alcohol) and gels prepared by freezing/thawing processes. In: Amjad, Press P, editors. Water Soluble Polymers. New York; 1998. p. 31-40. 
190. Yamaguchi H, Nakamura K, Numajiri S, Sugibayashi K, Morimoto Y. Swelling and mechanical properties of polyvinyl alcohol hydrogels. Int J Pharm. 1990;58(2):135-42.

191. Vidaurre A, Dueñas JMM, Estellés JM, Cortázar IC. Influence of Enzymatic Degradation on Physical Properties of Poly( $\varepsilon$-caprolactone) Films and Sponges. Macromol Symp [Internet]. WILEY-VCH Verlag; 2008 Aug 1;269(1):38-46.

192. Tang ZG, Black RA, Curran JM, Hunt JA, Rhodes NP, Williams DF. Surface properties and biocompatibility of solvent-cast poly[-caprolactone] films. Biomaterials. 2004;25(19):4741-8.

193. Amirian M, Chakoli AN, Cai W, Sui JH. In vitro degradation of poly(L-lactide)/poly(epsilon-caprolactone) blend reinforced with MWCNTs. Iran Polym J. 2012;21(3):165-74.

194. Wu F, Liu C, O'Neill B, Wei J, Ngothai Y. Fabrication and properties of porous scaffold of magnesium phosphate/polycaprolactone biocomposite for bone tissue engineering. Appl Surf Sci. 2012;258(19):7589-95.

195. Sarkar S, Isenberg BC, Hodis E, Leach JB, Desai TA, Wong JY. Fabrication of a Layered Microstructured Polycaprolactone Construct for 3-D Tissue Engineering. J Biomater Sci Polym Ed. 2008;19(10):1347-62.

196. Lin W-J, Lee H-G. Design of a microporous controlled delivery system for theophylline tablets. J Control Release. Netherlands; 2003 Apr;89(2):179-87.

197. Thadavirul N, Pavasant P, Supaphol P. Development of polycaprolactone porous scaffolds by combining solvent casting, particulate leaching, and polymer leaching techniques for bone tissue engineering. J Biomed Mater Res Part A [Internet]. 2014 Oct 1;102(10):3379-92.

198. Whang K, Thomas $\mathrm{CH}$, Healy KE. A novel method to fabricate biodegradable scaffolds. Polymer (Guildf). 1995;36:837-42. 
199. Ho M-H, Kuo P-Y, Hsieh H-J, Hsien T-Y, Hou L-T, Lai J-Y, et al. Preparation of porous scaffolds by using freeze-extraction and freeze-gelation methods. Biomaterials. England; 2004 Jan;25(1):129-38.

200. Lin W-J, Lu C-H. Characterization and permeation of microporous poly( $\varepsilon$-caprolactone) films. J Memb Sci [Internet]. 2002 Mar 31;198(1):109-18.

201. Lyu S, Untereker D. Degradability of Polymers for Implantable Biomedical Devices. Int J Mol Sci [Internet]. Molecular Diversity Preservation International (MDPI); 2009 Sep 11;10(9):4033-65.

202. Campillo-Fernández AJ, Pasto RS, Abad-Collado M, Bataille L, Gomez Ribelles JL, Meseguer Dueñas JM, et al. Future design of a new keratoprosthesis. Physical and biological analysis of polymeric substrates for epithelial cell growth. Biomacromolecules. 2007 Aug;8(8):2429-36.

203. Pérez Olmedilla M, Garcia-Giralt N, Monleón Pradas M, Ruiz P, Gómez Ribelles JL, Palou E, et al. Response of human chondrocytes to a non-uniform distribution of hydrophilic domains on poly (ethyl acrylate-co-hydroxyethyl methacrylate) copolymers. Biomaterials. 2006 Mar;27(7):1003-12.

204. Soria JM, Martínez Ramos C, Salmerón Sánchez M, Benavent V, Campillo Fernández A, Gómez Ribelles JL, et al. Survival and differentiation of embryonic neural explants on different biomaterials. J Biomed Mater Res A. 2006;79:495-502.

205. Rico P, Rodríguez Hernández JC, Moratal D, Altankov G, Monleón Pradas M, Salmerón Sánchez M. Substrateinduced assembly of fibronectin into networks: influence of surface chemistry and effect on osteoblast adhesion. Tissue Eng Part A. 2009;15(11):3271-81.

206. Gugutkov D, Altankov G, Rodríguez Hernández JC, Monleón Pradas M, Salmerón Sánchez M. Fibronectin activity on substrates with controlled -OH density. J Biomed Mater Res A. 2010 Jan;92(1):322-31. 
207. Salmerón Sánchez M, Rico P, Moratal D, Lee TT, Schwarzbauer J, García A. Role of material-driven fibronectin fibrillogenesis in cell differentiation. Biomaterials. Elsevier Ltd; 2011 Mar;32(8):2099-105.

208. Campillo-Fernández, AJ et al. Analysis of the biological response of endothelial and fibroblast cells cultured on synthetic scaffolds with various hydrophilic/hydrophobic ratios: influence of fibronectin adsorption and conformation. Tissue Eng Part A. 2009 Jun;15(6):1331-41.

209. Escobar, JL et al. Proliferation and differentiation of goat bone marrow stromal cells in 3D scaffolds with tunable hydrophilicity. J Biomed Mater Res B Appl Biomater. 2009 Oct;91(1):277-86.

210. Labet M, Thielemans W. Synthesis of polycaprolactone: a review. Chem Soc Rev. 2009;38:3484-504.

211. Diego RB, Estellés JM, Sanz J, García-Aznar JM, Salmerón Sánchez M. Polymer Scaffolds With Interconnected Spherical Pores and Controlled Architecture for Tissue Engineering: Fabrication, Mechanical Properties, and Finite Element Modelin. J Biomed Mater Res B Appl Biomater. 2007;81(2):448-55.

212. Diego RB, Olmedilla MP, Aroca AS, Gómez Ribelles JL, Monleón Pradas M, Gallego Ferrer G, et al. Acrylic scaffolds with interconnected spherical pores and controlled hydrophilicity for tissue engineering. J Mater Sci Mater Med. 2005;16(8):693-8.

213. Diego RB, Gomez Ribelles JL, Salmerón Sánchez M. Pore collapse during the fabrication process of rubber-like polymer scaffolds. J Appl Polym Sci. 2007;104(3):1475-81.

214. Chang HH, Yao L-C, Lin D, Cheng L-P. Preparation of microporous poly(VDF-co-HFP) membranes by templateleaching method. Sep Purif Technol. 2010;72(2):156-66.

215. Vallés Lluch A, Rodríguez-Hernández, J Gallego Ferrer G, Monleón Pradas M. Synthesis and characterization of poly(EMA-co-HEA)/SiO2 nanohybrids. Eur Polym J. 2010;46(7):1446-55. 
216. Vallés Lluch A, Poveda-Reyes S, Amorós P, Beltrán D, Monleón Pradas M. Hyaluronic acid-silica nanohybrid gels. Biomacromolecules. 2013;14(12):4217-25.

217. Wong BL, Sah RL. Effect of a focal articular defect on cartilage deformation during patello-femoral articulation. J Orthop Res. 2010;28(12):1554-61.

218. Guilak F, Ratcliffe A, Mow VC. Chondrocyte deformation and local tissue strain in articular cartilage: A confocal microscopy study. J Orthop Res. 1995;13(3):410-21.

219. Halonen KS, Mononen ME, Jurvelin JS, Töyräs J, Salo J, Korhonen RK. Deformation of articular cartilage during static loading of a knee joint--experimental and finite element analysis. J Biomech. 2014;47(10):2467-74.

220. Guo H, Maher SA, Torzilli PA. A biphasic multiscale study of the mechanical microenvironment of chondrocytes within articular cartilage under unconfined compression. J Biomech. 2014;47(11):2721-9.

221. Li LP, Korhonen RK, livarinen J, Jurvelin JS, Herzog W. Fluid pressure driven fibril reinforcement in creep and relaxation tests of articular cartilage. Med Eng Phys. 2008;30(2):182-9.

222. Ferry JD. Viscoelastic Properties of Polymers 3rd Edition. 1980.

223. Eckstein F, Lemberger B, Stammberger T, Englmeir KH, Reiser M. Patellar cartilage deformation in vivo after static versus dynamic loading. J Biomech. 2000;33(7):819-25.

224. Acosta Santamaría VA. Ingeniería de tejidos del cartílago articular: caracterización y modelado del comportamiento mecánico. University of Zaragoza; 2011.

225. Ateshian GA, Hung HA. The natural synovial joint: properties of cartilage. Proceeding Inst Mech Eng Part J- J Eng Tribol. 2005;220(J8):657-70. 
226. Buschmann MD, Soulhat J, Shirazi-Adl A, Jurvelin JS, Hunziker EB. Confined compression of articular cartilage: Linearity in ramp and sinusoidal tests and the importance of interdigitation and incomplete confinemen. J Biomech. 1998;31:171-8.

227. Quinn TM, Grodzinsky AJ. Longitudinal modulus and hydraulic permeability of poly (methacrylic acid) gels: effects of charge density and solvent content. Macromolecules. 1993;26(16):4332-8.

228. Ateshian GA, Warden WH, Kim JJ, Grelsamer RP, Mow VC. Finite deformation biphasic material properties of bovine articular cartilage from confined compression experiments. J Biomech. 1997;30:1157-64.

229. Lai MW, Mow VC. Drag-induced compression of articular cartilage during a permeation experiment. Biorheology. 1980;17:111-23.

230. Wheaton AJ, Dodge GR, Elliott DM, Nicoll SB, Reddy R. Quantification of cartilage biomechanical and biochemical properties via T1 $\rho$ magnetic resonance imaging. Magn Reson Med. 2005;54(5):1087-93.

231. Chin HC, Khayat G, Quinn TM. Improved characterization of cartilage mechanical properties using a combination of stress relaxation and creep. J Biomech. 2011;44(1):198201.

232. Gamboa-Martínez TC, Gómez Ribelles JL, Ferrer GG. Fibrin coating on poly (L-lactide) scaffolds for tissue engineering. J Bioact Compat Polym [Internet]. 2011 Aug 30;

233. Grodzinsky AJ, Levenston ME, Jin M, Frank EH. Cartilage tissue remodeling in response to mechanical forces. Annu Rev Biomed Eng. United States; 2000;2:691-713.

234. Cohen NP, Foster RJ, Mow VC. Composition and dynamics of articular cartilage: structure, function, and maintaining healthy state. J Orthop Sports Phys Ther. UNITED STATES; 1998 Oct;28(4):203-15. 
235. Wegener B, Schrimpf FM, Pietschmann MF, Milz S, BergerLohr M, Bergschmidt $P$, et al. Matrix-guided cartilage regeneration in chondral defects. Biotechnol Appl Biochem [Internet]. Blackwell Publishing Ltd; 2009 May 1;53(1):6370.

236. Suh JK, Scherping S, Mardi T, Steadman RJ, Woo SLY. Basic science of articular cartilage injury and repair. Oper Tech Sports Med [Internet]. 1995;3:78-86.

237. Holloway JL, Lowman AM, Palmese GR. Mechanical evaluation of poly(vinyl alcohol)-based fibrous composites as biomaterials for meniscal tissue replacement. Acta Biomater [Internet]. 2010 Dec;6(12):4716-24.

238. Gupta S, Sinha S, Sinha A. Composition dependent mechanical response of transparent poly(vinyl alcohol) hydrogels. Colloids Surfaces B Biointerfaces. 2010;78:1159.

239. Jurvelin JS, Buschmann MD, Hunziker EB. Mechanical anisotropy of the human knee articular cartilage in compression. Proc Inst Mech Eng H. 2003;217:215-9.

240. Salmerón Sánchez M, Monleón Pradas M, Gómez Ribelles $\mathrm{JL}$. Thermal transitions of benzene in a poly(ethyl acrylate) network. J Non Cryst Solids. 2002;(307):750-7.

241. Rault J, Lucas A, Neffati R, Monleón Pradas M. Thermal Transitions in Hydrogels of Poly(ethyl acrylate)/Poly(hydroxyethyl acrylate) Interpenetrating Networks. Macromolecules. 1997;30(25):7866-73.

242. Vikingsson L, Gallego Ferrer G, Gómez-Tejedor JA, Gómez Ribelles JL. An in vitro experimental model to predict the mechanical behaviour of macroporous scaffolds implanted in articular cartilage. J Mech Behav Biomed Mater. Elsevier; 2014;32:125-31.

243. Frank EH, Grodzinsky AJ. Cartilage electromechanics II-a continuum model of cartilage electrokinetics and correlation with experiments. J Biomech. 1987;20(6):629-39. 
244. Chen AC, Bae WC, Schinagl RM, Sah RL. Depth- and strain-dependent mechanical and electromechanical properties of full-thickness bovine articular cartilage in confined compression. J Biomech. 2001;34(1):1-12.

245. Alves NM, Mano JF, Balaguer E, Meseguer Dueñas JM, Gómez Ribelles JL. Glass transition and structural relaxation in semi-crystalline poly(ethylene terephthalate). A DSC study. Polymer (Guildf). 2002;43(15):4111-22.

246. Wang Y, Gómez Ribelles JL, Salmerón Sánchez M, Mano JF. Morphological contribution to Glass Transition in poly(Llactic acid). Macromolecules. 2005;38:4712-8.

247. Blasia P, D'Souzab S, Selminc F, DeLucad PP. Plasticizing effect of water on poly(lactide-co-glycolide). J Control Release. 2005;108(1):1-9.

248. Love AE. Treatise on the Mathematical Theory of Elasticity. Dover Book. New York: Dover Publications; 1927.

249. Ewing JA. On hysteresis in the relation of strain to stress. Newcastle-upon-Tyne; 1889.

250. Panadero JA, Vikingsson L, Gómez Ribelles JL, Sencadas $\mathrm{V}$, Lanceros-Mendez S. Fatigue prediction in fibrin poly- $\varepsilon-$ caprolactone macroporous scaffolds. J Mech Behav Biomed Mater [Internet]. 2013 Dec;28(0):55-61.

251. Jeong CG, Hollister SJ. Mechanical, permeability, and degradation properties of 3D designed poly $(1,8$ octanediolco-citrate) scaffolds for soft tissue engineering. J Biomed Mater Res B Appl Biomater. United States; 2010 Apr;93(1):141-9. 